Biological Effects of Power Frequency Electric and Magnetic Fields

May 1989

NTIS order \#PB89-209985
Biological Effects of Power Frequency Electric and Magnetic Fields

Background Paper 


\title{
Biological Effects of Power Frequency Electric and Magnetic Fields
}

\author{
Background Paper
}

This background paper was performed as part of OTA'S assessment of Electric Power Wheeling and Dealing:

Technological Considerations for Increasing Competition

\author{
Prepared for OTA by: \\ Indira Nair \\ M. Granger Morgan

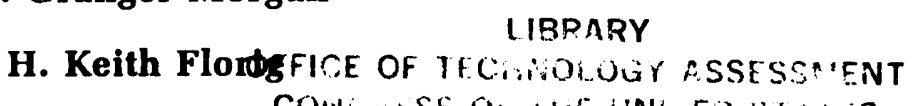 \\ COINUAESS Or IIAE UNIIEO SIAICS \\ WASHINGTON, D. C. 20510
}

Department of Engineering and Public Policy

Carnegie Mellon University

Pittsburgh, PA 15213

NOTE: OTA makes this background paper available for the use of readers desiring a more detailed or technical discussion of the issue than can be accommodated in OTA's final report. As an OTA background paper, it has not been reviewed or approved by the Technology Assessment Board. The findings and conclusions expressed in this report are those of the authors and do not necessarily reflect the views of OTA, the electric power project advisory panel, or the Technology Assessment Board. 


\section{Recommended Citation:}

U.S. Congress, Office of Technology Assessment, Bilogical Effects of Power Frequency Electric \& Magnetic Fields-Background Paper, OTA-BP-E-53 (Washington, DC: U.S. Government Printing Office, May 1989).

\section{Library of Congress Catalog Card Number 89-600708}

For sale by the Superintendent of Documents

U.S. Government Printing Office, Washington, DC 20402-9325

(order form can be found in the back of this report) 
Foreword

This background report responds to a request by the Subcommittee on Water and Power Resources of the House Committee on Interior and Insular Affairs. The subcommittee asked that OTA review the health effects of high-voltage transmission lines. To provide background information for its assessment on electric power wheeling, OTA contracted with the Carnegie-Mellon University. This report was used in the preparation of OTA's final assessment Electric Power Wheeling and Dealing: Technological Considerations for Increasing Competition.

For about two decades, there has been some concern about the health effects of electric and magnetic fields produced by transmission lines. Recent studies have heightened this concern. Health effects research is still preliminary and inconclusive, but a growing number of studies suggest that under certain circumstances even relatively weak electric and magnetic fields can produce biologic changes. This report discusses the present state of knowledge on the health effects of low-frequency electric and magnetic fields and describes current U.S. funding levels and research programs. Also, the report provides information on regulatory activity, including existing and proposed field exposure standards.

OTA acknowledges the generous help of the reviewers who gave their time to ensure the accuracy and completeness of this report. In particular, OTA thanks the project's distinguished advisory panel and workshop participants.

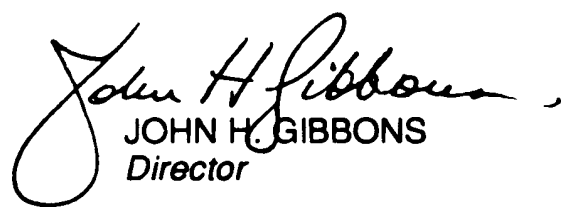

iii 


\title{
OTA Project Staff-Electric Power Wheeling and Dealing: Technological Considerations for Increasing Competition
}

\author{
Lionel S. Johns, Assistant Director, OTA \\ Energy, Materials, and International Security Division \\ Peter D. Blair, Energy and Materials Program Manager \\ Alan T. Crane, Project Director \\ Karen Larsen, Senior Analyst \\ Robin Roy, Analyst \\ Joanne Seder, Analyst \\ Administrative Staff \\ Lillian Chapman Linda Long Phyllis Brumfield \\ Contributor \\ Hellen Gelband \\ Reviewers \\ Daniel Driscoll, Department of Public Service, State of New York \\ William W. Lowrance, The Rockefeller University \\ Joseph Norton, Department of Environmental Regulation, State of Florida \\ Lee Rosen, W/L Associates \\ David Savitz, University of North Carolina \\ Nancy Wertheimer, Boulder, Colorado
}




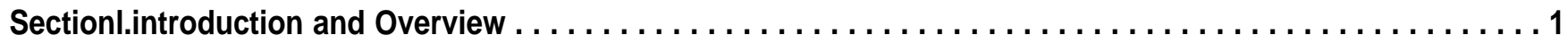

Section 2. Sources and Nature of Fields and Exposure $\ldots \ldots \ldots \ldots \ldots \ldots \ldots \ldots \ldots \ldots \ldots \ldots \ldots \ldots \ldots$

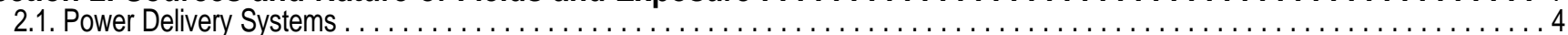

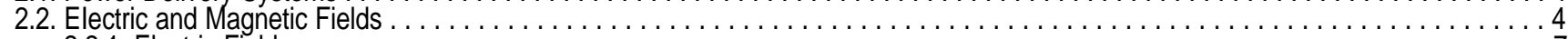

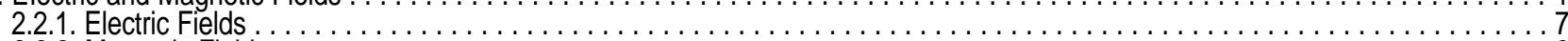

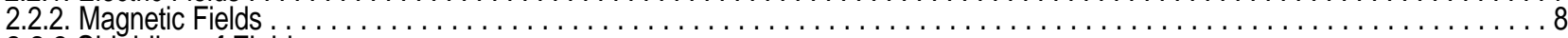

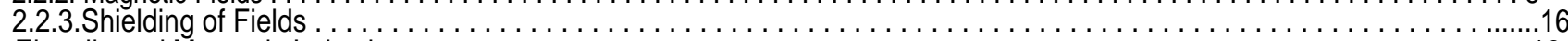

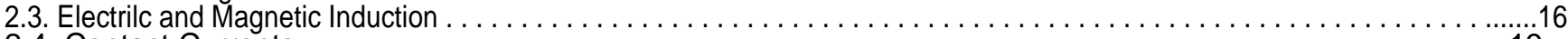

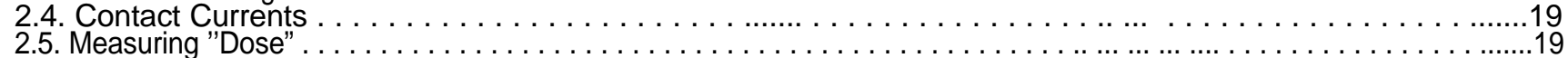

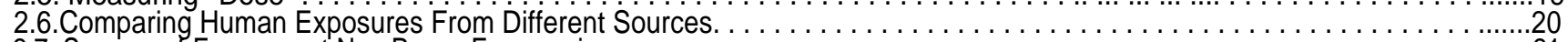

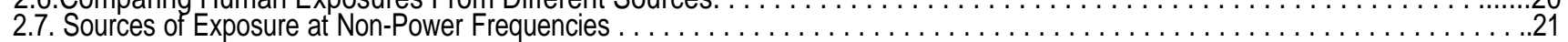

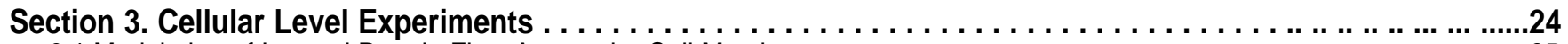

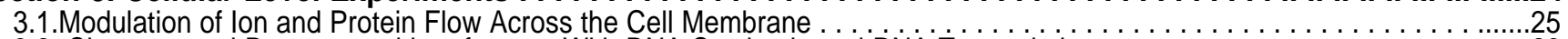

3.2. Chromosomal Damage and interference With DNA Synthesis and ṘNA Ṫanscription $\ldots \ldots \ldots \ldots \ldots \ldots$

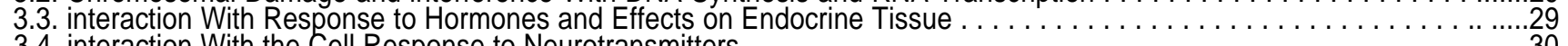

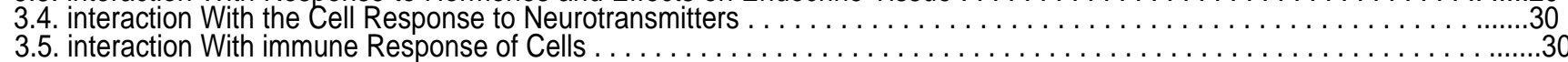

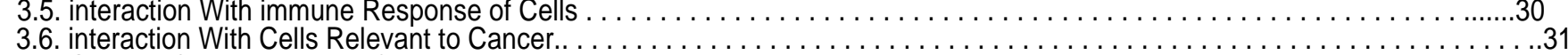

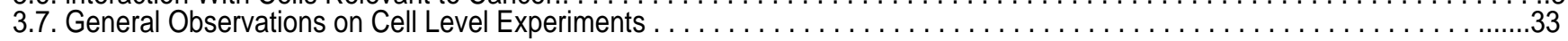

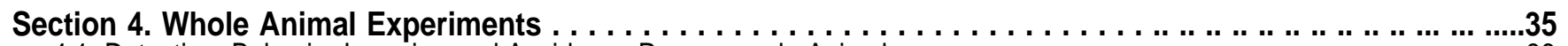

4.1. Detection, Behavior Learning and Avoidance Responses In Animals $\ldots \ldots \ldots \ldots \ldots \ldots \ldots \ldots$

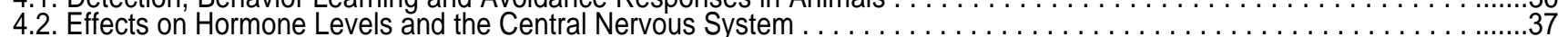

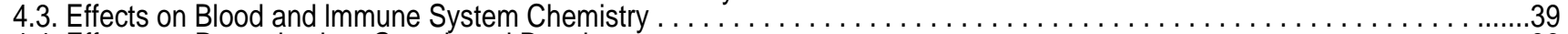

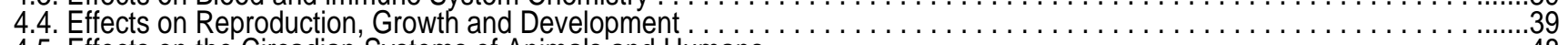

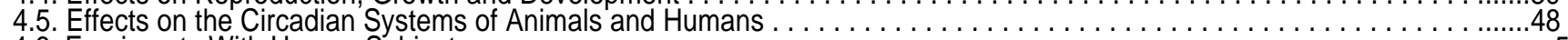

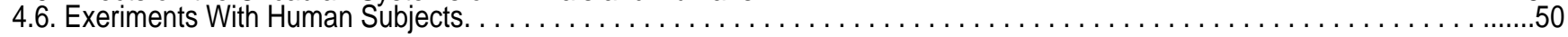

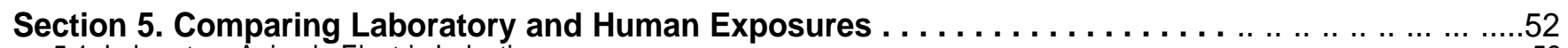

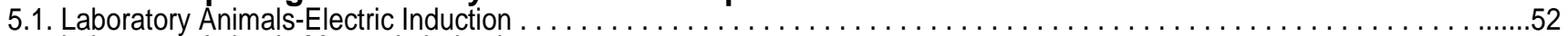

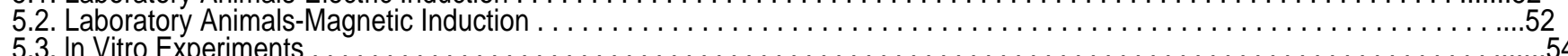

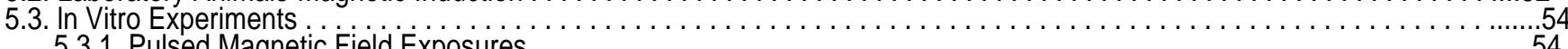

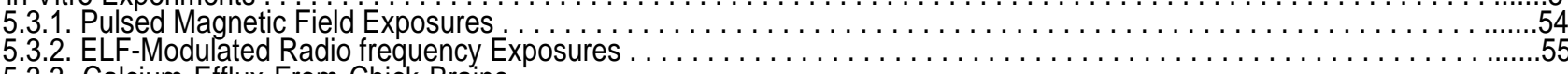

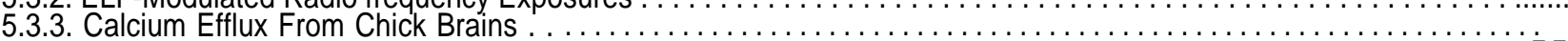

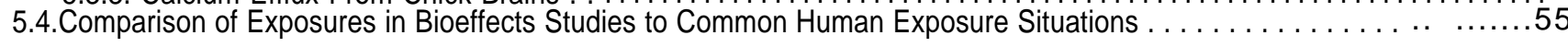

Section 6. Cancer and Electromagnetic Fields: Epidemiological Studies . . . . . . . . . . . . . . . . 57

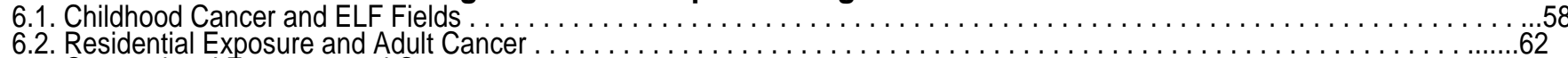

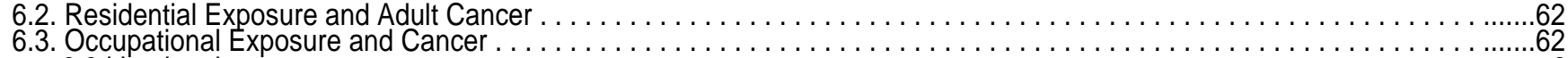

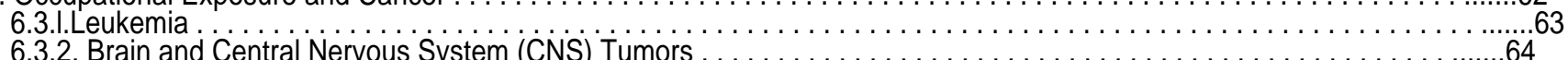

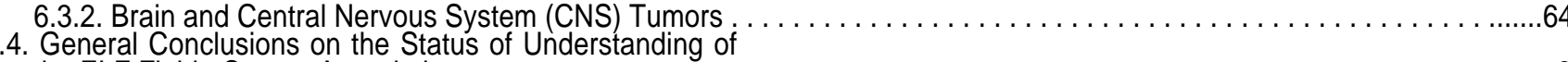

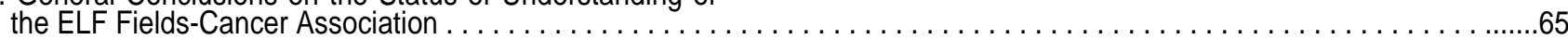

Section 7. General Conclusions About Biological Effects of ELF Fields

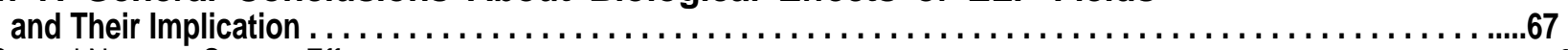

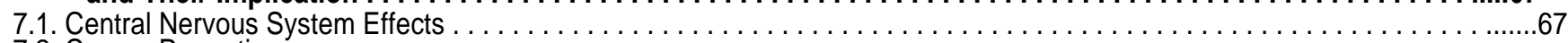

7.2. Cancer Promotion

Section 8. Major Programs and Funding Levels for ELF Bioeffects Research ... ... ..........69

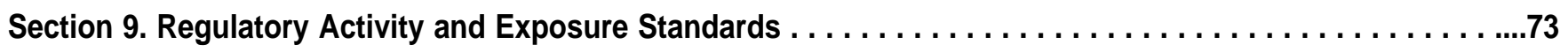

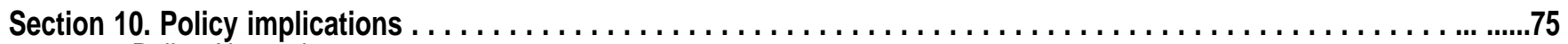

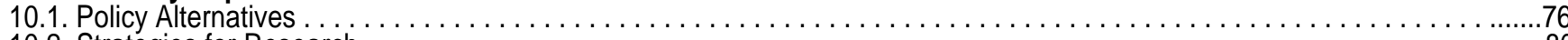

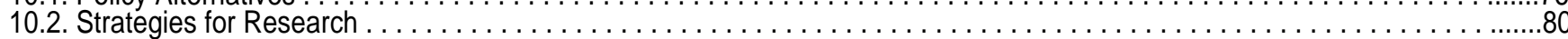

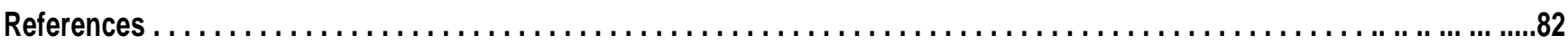

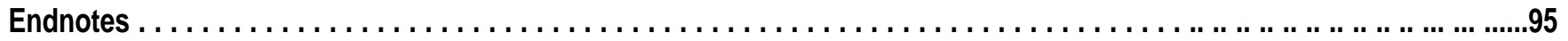




\section{Introduction and Overview}

Electric and magnetic fields produced by electric power systems have recently been added to the list of environmental agents that are a potential threat to public health. This paper describes peoples' exposures to fields from power systems and other sources (Section 2), reviews existing scientific evidence on the biological effects of these fields (Sections 3 through 7), presents a history of research support and of regulatory activity (Sections 8 and 9 ), and discusses problems and alternatives in regulatory action (Section 10$)$.

The electric power that is used in our homes, offices and factories uses AC or alternating current. This is in contrast to the DC or direct current that is produced by batteries. An alternating current does not flow steadily in one direction. It alternates back and forth. The power used in North America alternates back and forth 60 times each second. This is called 60 hertz $(\mathrm{Hz})$ power. In Europe and some other parts of the world the frequency of electric power is 50 hertz rather than $60 \mathrm{~Hz}$.

There are electric and magnetic fields wherever there is electric power. This means that there are fields associated with large and small powerlines, wiring and lighting in homes and places of work, and all electrical appliances. These fields are created by the electric charges that are pumped into the power system by electric power generating stations. Electric fields arise from the amount of that charge and magnetic fields result from the motion of that charge. Taken together, these fields are often referred to as electromagnetic fields. The electric and magnetic fields created by power systems oscillate with the current. That is why fields around power systems are called power-frequency or 60 hertz fields. A more complete description of the electromagnetic fields from power systems is presented in Section 2.

Public concerns about power-frequency fields first emerged in the late 1960s as power companies turned increasingly to extra high voltage (EHV) transmission lines to handle large increases in electricity use. EHV lines carry electric power with lower energy losses and with smaller land usage than multiple lower-voltage lines with the same power-delivery capacity. Public attention to EHV transmission lines focused first on the aesthetic impact of their large towers, on the aesthetic and ecological impacts of their rights-of-way, and on various nuisance effects created by their strong electric fields. These nuisance effects include audible noise, TV/radio interference, and induced shocks that can occur when a person standing beneath an EHV line touches a large ungrounded metal object such as a truck or farm vehicle. By the early 1970s, the American National Standards Institute had issued voluntary standards to address nuisance effects. The first evidence that power-frequency fields might have a direct effect on human health appeared in 1972 when Soviet investigators reported that workers in Soviet EHV switchyards suffered from a number of nonspecific ailments [Korobkova 72]. Although these reports were greeted with much skepticism by western scientists, they served to stimulate public concern. By the midseventies, health effects had become a central issue in transmission line siting hearings in several states.

There are two reasons why conventional wisdom has until recently held that the fields associated with power systems could pose no threat to human health. First, there is no significant transfer of energy from power-frequency fields to biological systems. Unlike X-rays (i.e. ionizing radiation), powerfrequency fields do not break chemical bonds. Unlike microwaves (i.e. non-ionizing radiation), powerfrequency fields cannot cause significant tissue heating. Second, all cells in the body maintain large natural electric fields across their outer membranes. These naturally occurring fields are at least 100 times more intense than those that can be induced by exposure to common power-frequency fields. 
However, despite the low energy of power-frequency fields and the very small perturbations that they make to the natural fields within the body, studies over the last fifteen years have demonstrated unequivocally that under certain circumstances, the membranes of cells can be sensitive to even fairly weak externally imposed low frequency electromagnetic fields. Extremely small signal changes can trigger major biochemical responses critical to the functioning of the cell [Adey 81, Adey 84, Adey 87]. This should perhaps have come as no surprise, as cells, especially those in the nervous system, make use of complex electrochemical processes in their normal function. The ability of some animals including eels, sharks, and pigeons to detect extremely weak ELF fields and use them for homing and finding prey clearly demonstrates that at least some specialized cells can be exquisitively sensitive to such fields.

[Fessard 74, Gould 82]. Among the responses demonstrated in laboratory studies using animal cells and tissue are:

- modulation of ion flows;

- interference with DNA synthesis and RNA transcription;

- interaction with the response of normal cells to various agents and biochemical such as hormones, neurotransmitters, and growth factors;

- interaction with the biochemical kinetics of cancer cells.

Even when effects are demonstrated consistently on the cellular level in laboratory experiments, it is hard to predict whether and how they will affect the whole organism. Processes at the individual cell level are integrated through complex mechanisms in the animal. When a process in the cell is lightly perturbed by an external agent such as an ELF field, other processes may compensate for it so that there is no overall disturbance to the organism. Some perturbations may be within the ranges of disturbances that a system can experience and still function properly. This difficulty in extrapolating cellular level effects to predict the existence or severity of possible public health effects, together with the absence of any large-scale and obvious public health effect associated with electrification, are two arguments advanced during the last decade in support of the claim that there is no need for concern about possible public health effects from exposure to power-frequency fields.

Another problem in deducing possible health effects from cellular level effects has been the lack of a theoretical model to explain and understand the detailed mechanism of interaction. ELF fields affect the cell via the cell membrane. Cell membrane biology is still in its infancy although this area of molecular biology has made great strides in the past few years. Until recently, there was not enough understanding to even advance hypotheses on the potential mechanisms by which ELF fields may cause significant perturbations in cell and organ functions. Hypotheses are now being advanced but are still at a speculative stage [Adey 86, Smith 87, Liboff 86].

As we discuss in Section 3, findings at the cellular level display considerable complexity including resonant responses (or, "windows") in frequency and field strength, complex time dependencies, and dependence on the ambient DC magnetic field created by the earth. For these reasons, ELF fields appear to be an agent to which there is no known analog. Many lessons learned from environmental hazards such as chemical agents (PCB, vinyl chloride, benzene, etc.) or physical agents (ionizing radiation, asbestos etc.) may not directly apply to ELF fields. This is because in the case of fields it is not yet clear what measures of exposure or "dose" are relevant. In contrast to more familiar environmental agents where "if some of it is bad, more of it is worse", it may not be safe to assume that if ELF field 
exposure leads to health risks, exposure to stronger fields or exposure for longer periods is worse than exposure to weaker fields or brief periods.

In addition to cellular studies, whole animal and human experiments have examined five general categories of effects:

1. General effects such as detection, avoidance and behavior response and development and learning of animals, and moods of humans;

2. Effects on externally measured physical parameters such as growth and birthweight, respiration, heartbeat rate, and temperature rhythms;

3. Effects on specific biochemical such as hormones that are responsible for the maintenance, regulation and control of general physiological and psychological functions ; for response to environmental stressors; for growth and development; and, for triggering special responses such as sexual function, and fetal and newborn nourishment;

4, Effects on circadian rhythms of animals and humans; and,

5. Effects in the epidemiology of cancer, particularly leukemia and brain cancer.

Several authors and scientific advisory panels have reviewed the effects literature. [Adey 86, Adey 87, AIBS 85, Carstensen 87, Florida 85, Grandolfo 86, Lee 86, NYSPLP 87, Sheppard 83, West 86, WHO 84]. In summary, the results are complex and inconclusive. There have been many "negative" experiments, that is, experiments that have looked for effects but not found any difference between biological systems that have been exposed to fields and those that have not. However, the growing number of positive findings have now clearly demonstrated that under specific circumstances even weak low-frequency electromagnetic fields can produce substantial changes at the cellular level, and in a few experimental settings, effects have also been demonstrated at the level of the whole animal.

Epidemiological evidence, while controversial and subject to a variety of criticisms, is beginning to provide a basis for concern about risks from chronic exposure. Some observers find this epidemiological evidence more persuasive in light of the clear evidence of effects that is available at the cellular level, but others insist on treating the evidence from these two areas as separate.

As recently as a few years ago, scientists were making categorical statements that on the basis of all available evidence there are no health risks from human exposure to power-frequency fields. In our view, the emerging evidence no longer allows one to categorically assert that there are no risks. But it does not provide a basis for asserting that there is a significant risk.

If exposure to fields does turn out to pose a health risk, it is unlikely that high voltage transmission lines will be the only sources of concern. Power-frequency fields are also produced by distribution lines, wall wiring, appliances, and lighting fixtures. These non-transmission sources are much more common than transmission lines and could play a far greater role than transmission lines in any public health problem. 


\section{Sources and Nature of Fields and Exposure}

\subsection{Power Delivery Systems}

Power lines are characterized by voltages and currents. Voltage is a measure of the electric potential energy that makes electric charges flow through a circuit. Current is a measure of the rate at which electrical charges flow in a power line or wire. The amount of power that a line transmits is simply the product of its voltage and current. Power systems are designed so that line voltage is held relatively constant over time while currents are permitted to rise and fall with power demand.

The various stages of the system that is used to move electric power from the electric generator to the end user are depicted in Figure 2-1. Electric generators in power stations produce electric power at about 20,000 volts (20 kilovolts or $20 \mathrm{kV}$ ). Large "step-up" transformers are used to increase the voltage for efficient long distance power transfer over high voltage transmission lines to load centers.

Transmission lines operate at voltages up to $785 \mathrm{kV}$ and carry current of up to 2000 Amperes (or Amps). They are usually mounted on metal or wooden structures up to 50 meters in height. Transmission lines terminate at substations where step-down transformers transfer power to lower-voltage distribution lines. Distribution lines deliver power locally through load centers to individual users. Residential distribution systems are comprised of two different circuits: 1$)$ a high voltage $(5-35 \mathrm{kV})$ or "primary" circuit that aelivers power from the substation to a local pole-mounted or underground distribution transformer and 2) a low voltage or *'secondary" circuit that delivers power from the local transformer to the home. The voltage of the secondary side is low enough (1 15/230 V) to allow appliances, lighting, and other electrical loads in the home to be operated safely. Commercial and industrial installations often have their own step-down transformers and so obtain their power directly from distribution primaries. Distribution primaries carry currents of up to 900 Amps. $115 / 230$ volt wall-wiring in homes is typically designed to carry currents of up to 30 Amps.

The amount of power lost in the wires during transmission and distribution can be reduced by increasing the voltage at which these lines operate. Transmission and primary distribution voltages have therefore increased over the years as rapidly as high voltage technology has allowed. Figure 2-2 shows this trend for transmission lines.

The length of transmission and distribution lines in service have also increased steadily over the last century in response to increases in population and per capita demand for electricity. Today, there are about 350,000 miles of transmission line and about 2 million miles of distribution line in the U.S. [Minner 87, USDOE 83].

\subsection{Electric and Magnetic Fields}

Although "electric and magnetic fields" may sound mysterious or ominous to some people, scientists have had a good understanding of them since the nineteenth century. Electric and magnetic fields arise from many natural sources. They appear throughout nature and in all living things. Electromagnetic forces are responsible for holding atoms together in molecules of chemical compounds. Processes in the atmosphere produce large static electric fields at the surface of the earth, thunderclouds produce lightning as they discharge stored up charge, and processes in the earth's core give rise to a magnetic field which makes navigation by compass possible. Modern devices such as TV, radio, and microwave ovens depend on electric and magnetic fields for their operation. There are numerous technological uses for electric and magnetic fields. 


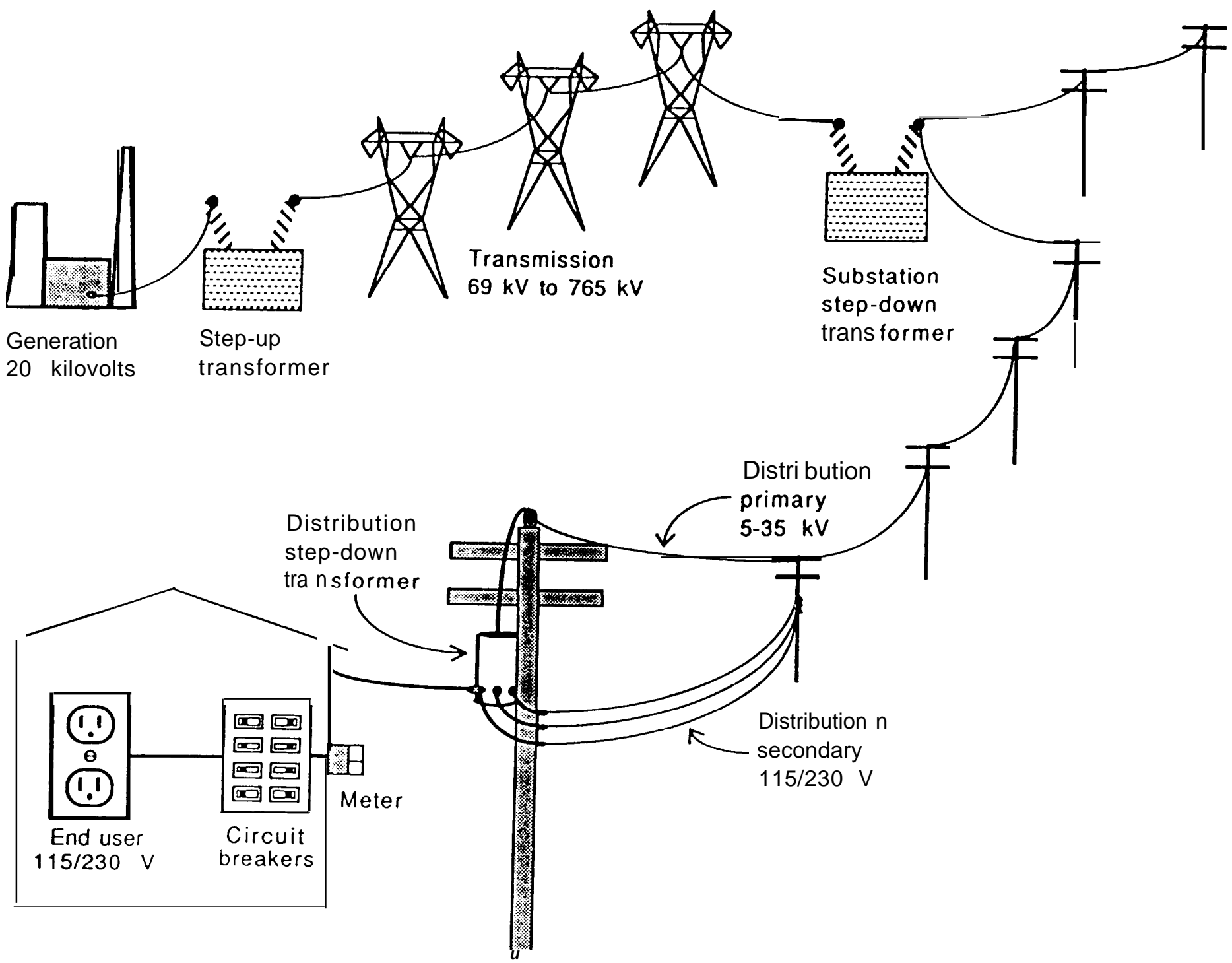

Figure 2-1: Schematic illustration (prepared by the authors) of the stages in the system used to transfer power from the generator via transmission and distribution lines to an end user. Power on transmission lines and distribution systems is delivered using sets of three wires. While the voltages on all three wires oscillate at power-frequency, the oscillations are not "in phase" with one another. As the voltage on one wire is peaking, the voltage on one of the others is one-third of a cycle ahead and the voltage on the other wire is one-third of a cycle behind. For this reason, the three wires are referred to as the three phases of the power network. Although commercial and industrial facilities use three-phase power to run large motors and other heavy loads, the $115 \mathrm{~V}$ power in homes is generally supplied by just a single phase. Utility a)companies try to connect equal numbers of houses to each phase of a residential distribution network in order to balance the load across the phases. 


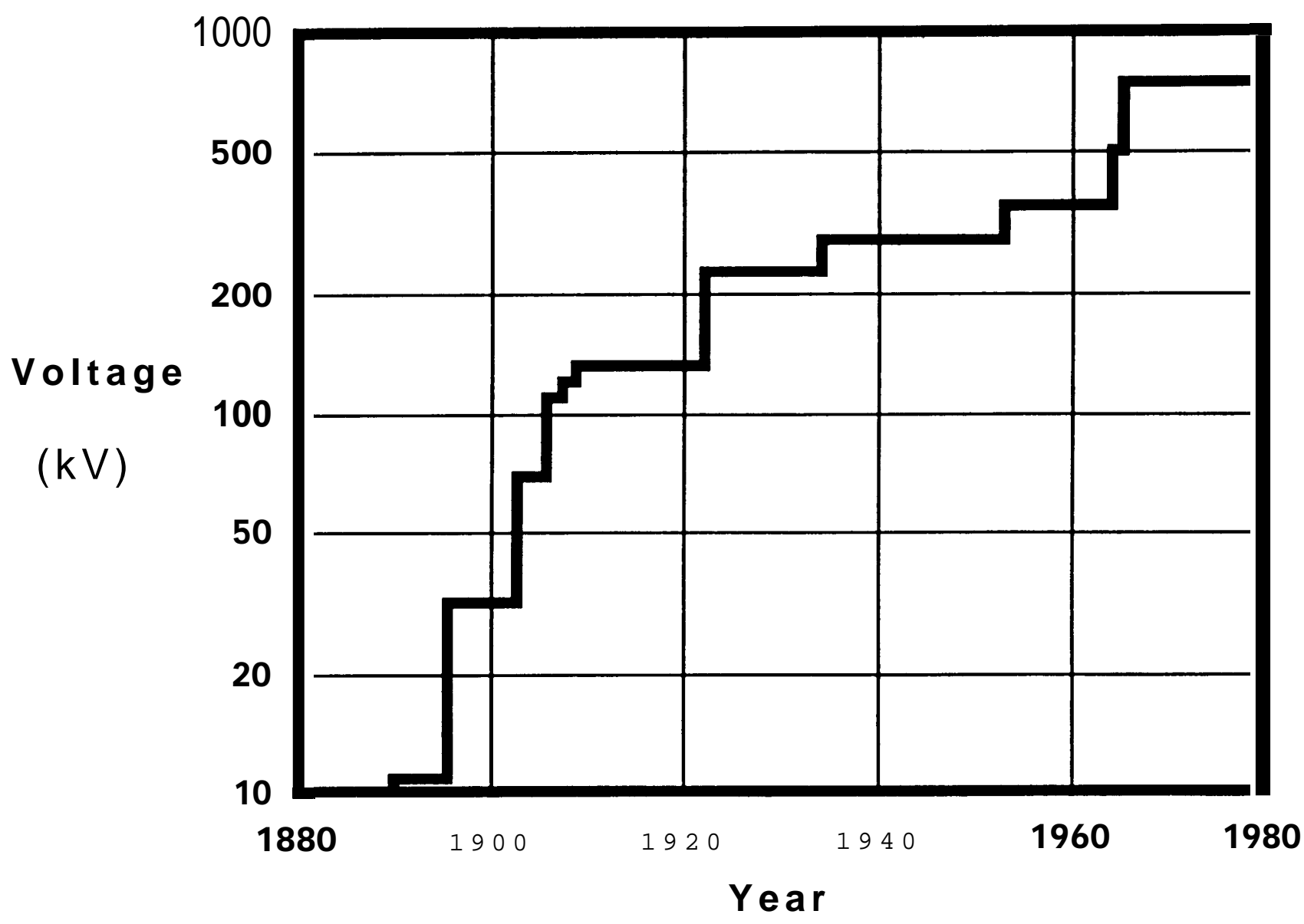

Figure 2-2: Highest $60 \mathrm{~Hz}$ transmission voltage in North America. From [Ellert 82].

There are two types of electric and magnetic fields, those that travel or propagate long distances from their source (also called electromagnetic waves) and those that are confined to the immediate vicinity of their source. At distances that are close to a source compared to a wavelength, ' fields are primarily of the confined type. Confined fields decrease in intensity much more rapidly with distance from their source than do propagating fields. Propagating fields dominate, therefore, at distances that are far from the source compared to a wavelength. The fields to which most people are exposed from radio broadcast antennas are examples of propagating fields since these source are generally much more than one wavelength (1-1 00 meters) removed from inhabited areas. The power-frequency fields that people encounter are of the non-propagating type because power lines and appliances are much closer to people than one $60 \mathrm{~Hz}$ wavelength (several thousand kilometers). Only a very miniscule portion of the energy in power lines goes into propagating fields. Because the power-frequency fields of public health concern are not of the propagating type, it is technically inappropriate to refer to them as "radiation".

\footnotetext{
'A wavelength is the distance that a propagating field travels during one oscillatory cycle. For fields in air this distance is $\mathrm{c} / \mathrm{f}$ where $c$ is the velocity of light and $f$ is the frequency of the oscillating field.
} 


\subsubsection{Electric Fields}

Many fundamental particles such as electrons and protons carry an electric charge. Protons carry a positive charge and electrons carry a negative charge. Charges of the same sign repel one another whereas charges of opposite sign attract each other. Most of the time most objects have almost exactly the same number of electrons as they do protons. So the effect of the charges cancel and the object has no overall charge. The "static electricity" of clinging clothes is an example of the electric forces that result when objects acquire a small excess of positive or negative charges (this happens in the drier when clothes pick up or lose electrons from one another as they rub together). The "electric field" of a charged object is merely a description of the electric force that the object is capable of exerting on other charges brought into its vicinity. The intensity of the electric field is proportional to the magnitude of this force. Electric fields are represented graphically by sets of "field lines". The direction of the field lines indicates the direction of the electric force at any point. The density or spacing of the lines corresponds to the intensity of the field. Figure 2-3 shows the electric field that exists between two equal and opposite electric charges. Electric field intensity is measured in units of volts per meter $(\mathrm{V} / \mathrm{m})$. One thousand volts per meter is a kilovolt per meter $(\mathrm{kV} / \mathrm{m})$. Field intensities are sometimes referred to as "field values" or simply as "fields".

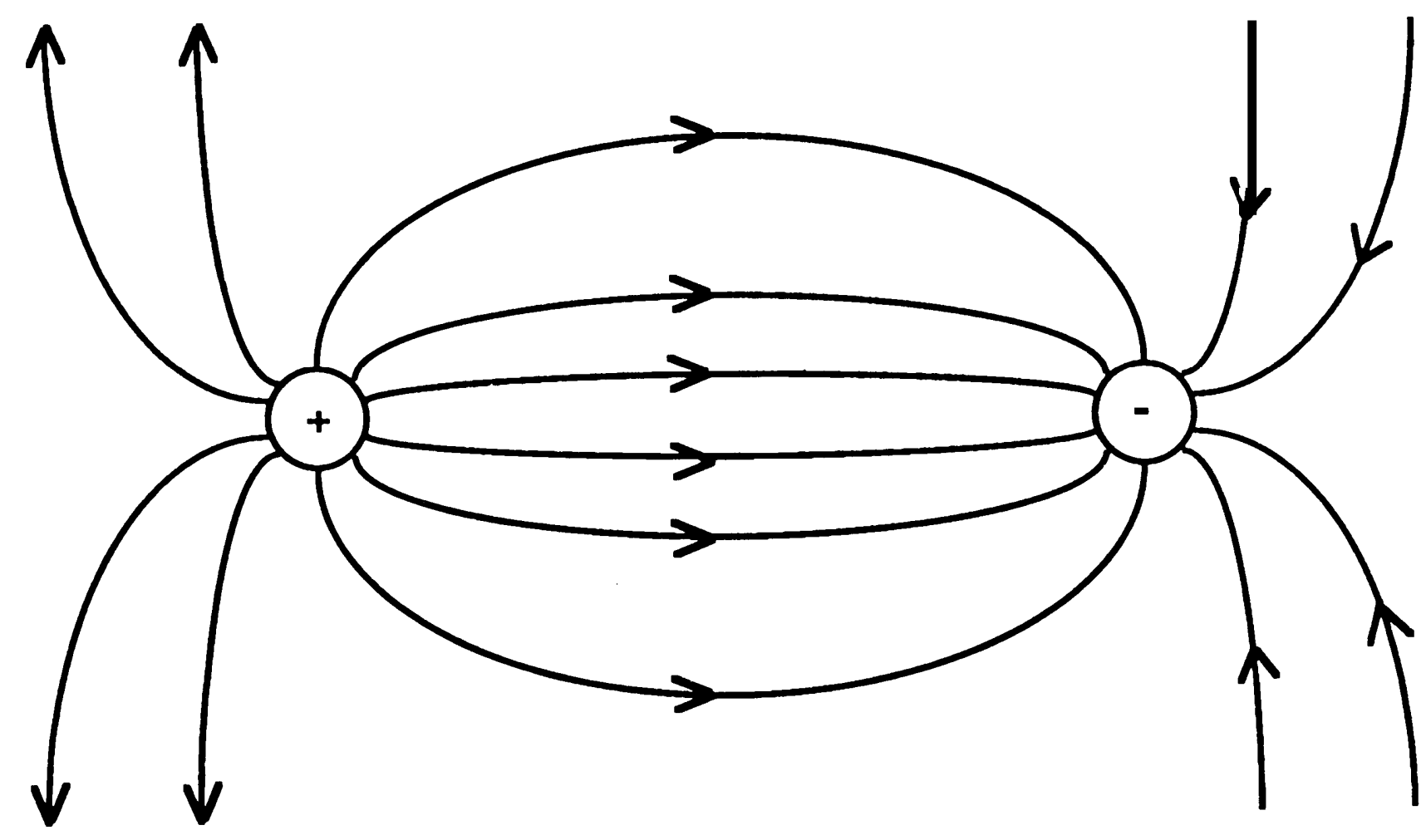

Figure 2-3: The electric field of two equal but opposite charges. A small positively charged particle placed somewhere in this field will experience a force in the direction-of the-local field line (note arrows). The strength of the force is proportional to the spacing between the field lines (field lines closer together means a higher field and thus a larger force on small charged particle).

The electric fields of power lines, wall wiring, and appliances are produced by electric charges that 
are "pumped" onto the wires by electric generators. Because the charge in the wire changes from positive to negative at power-frequency, the associated electric fields are dynamic. Dynamic fields can be depicted by taking some time-average measure of their intensity and direction. The most common convention is to use the "root-mean-square" field. Figure 2-4 is a representation of the power-frequency electric field of a household coffee maker obtained using the root-mean- square.

As with fields from other power-frequency sources, the electric field from a coffee maker loses intensity rapidly with distance. Figure $2-5$ shows how electric field strength changes with distance for electric fields from EHV transmission lines, distribution lines, and typical appliances.

\subsubsection{Magnetic Fields}

In the nineteenth century, scientists discovered that a current-carrying wire exerts a force on any charged particle moving nearby. This force was called the magnetic force. Its magnitude is proportional to the current in the wire and the velocity and charge of the moving particle. The magnetic field is a mathematical means of representing the magnetic force. Like electric fields, magnetic fields are represented graphically by sets of lines as shown in Figure 2-6.

There are several different units used to describe magnetic fields. The proper unit of magnetic field intensity is the Ampere per meter (analogous to the $\mathrm{V} / \mathrm{m}$ for electric fields). Often, magnetic field strength is indicated by a related quantity called the magnetic flux density which is the number of field lines that cross a unit of surface area. The unit of magnetic flux density that is encountered most often in the power-frequency literature is the gauss $(G)$. Sometimes, the magnetic flux density is given in tesla (T). There are 10,000 gauss in each tesla. For fields in air or in biological tissues, the magnetic flux density in gauss is $1 / 80$ th of the magnetic field intensity in $\mathrm{A} / \mathrm{m}$. The gauss and tesla are large units. Sixty hertz magnetic fields are commonly reported in thousandths of a gauss or milligauss (mG).

Like power-frequency electric fields, magnetic fields from power systems are dynamic and are generally described by some time-averaged quantity such as the root mean square. Figure 2-7 shows the root mean square field of a household coffee maker.

Magnetic field intensity drops off rapidly with distance. Figure 2-8 shows this relationship for magnetic fields from EHV transmission lines, distribution lines, and typical appliances. The magnetic fields around many appliances are stronger than the magnetic fields under either transmission or distribution lines. Appliance fields typically fall off faster with distance, however, than do fields from overhead powerlines. This results from the fact that appliances are less extended in space than are long power lines.

Electric and magnetic fields produced by power lines and other sources can be either measured using a "field meter" or calculated given information on voltage and current. For transmission lines, such calculations can be quite accurate. Published reports describing fields from various sources are listed in Table 2-1.

Recent epidemiological studies relating the incidence of certain cancers to magnetic fields in the household environment [Wertheimer 79, Wertheimer 82, Savitz 87a, Stevens 871 have created a growing need to understand the various sources of magnetic fields in the home. These sources include 1 ) appliances, 2) wall wiring, 3) ground currents in plumbing, gas lines, and steel girders, and 4) overhead and underground distribution wires [Barnes 87]. The most intense magnetic fields in the home are found 


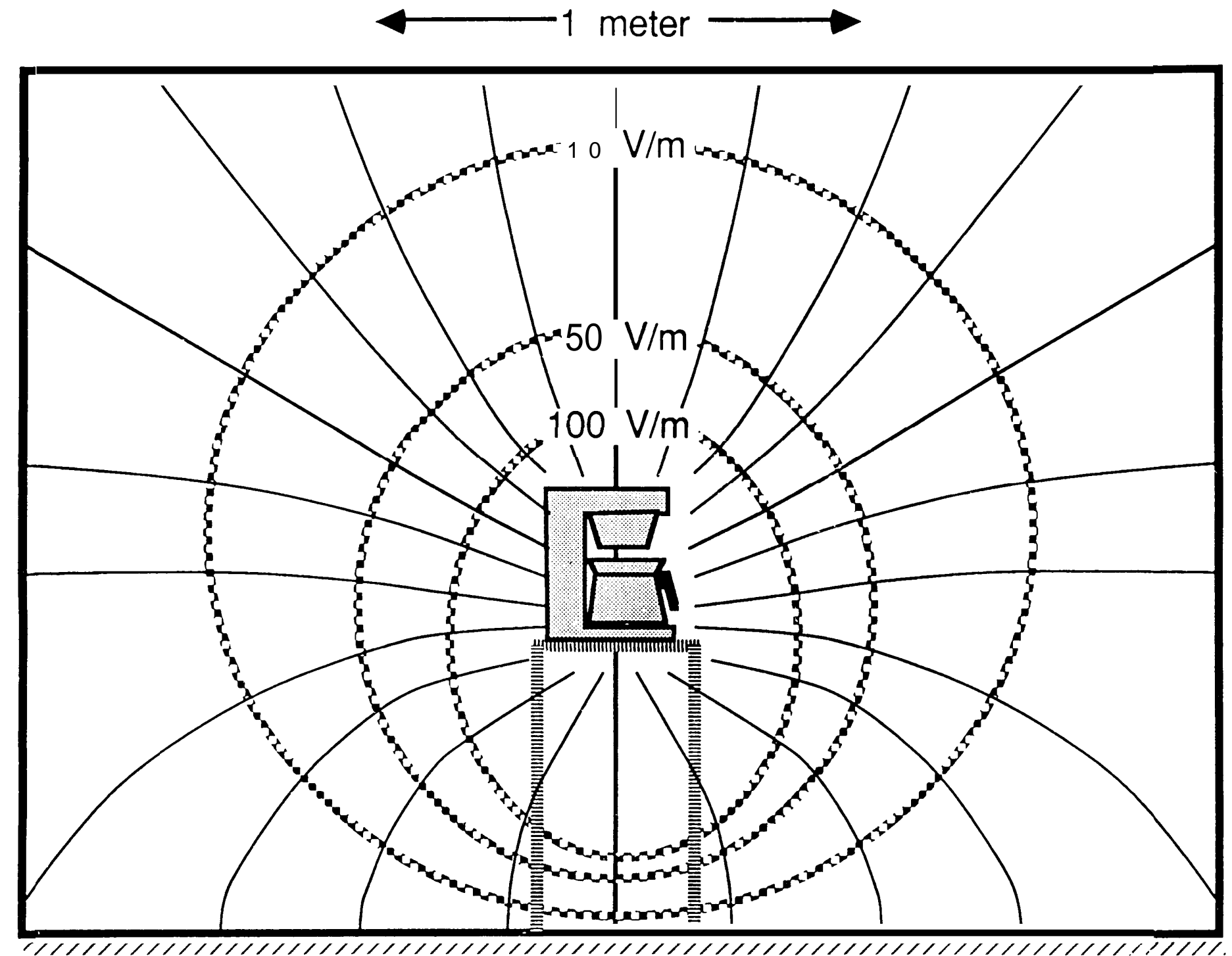

GROUND

Figure 2-4: Time averaged (root mean square) electric field of a $115 \mathrm{~V}$ coffee maker (solid lines).

Dotted lines show surfaces of equal field intensity. These differ from equipotential surfaces.

Adapted from [Florig 86]. 


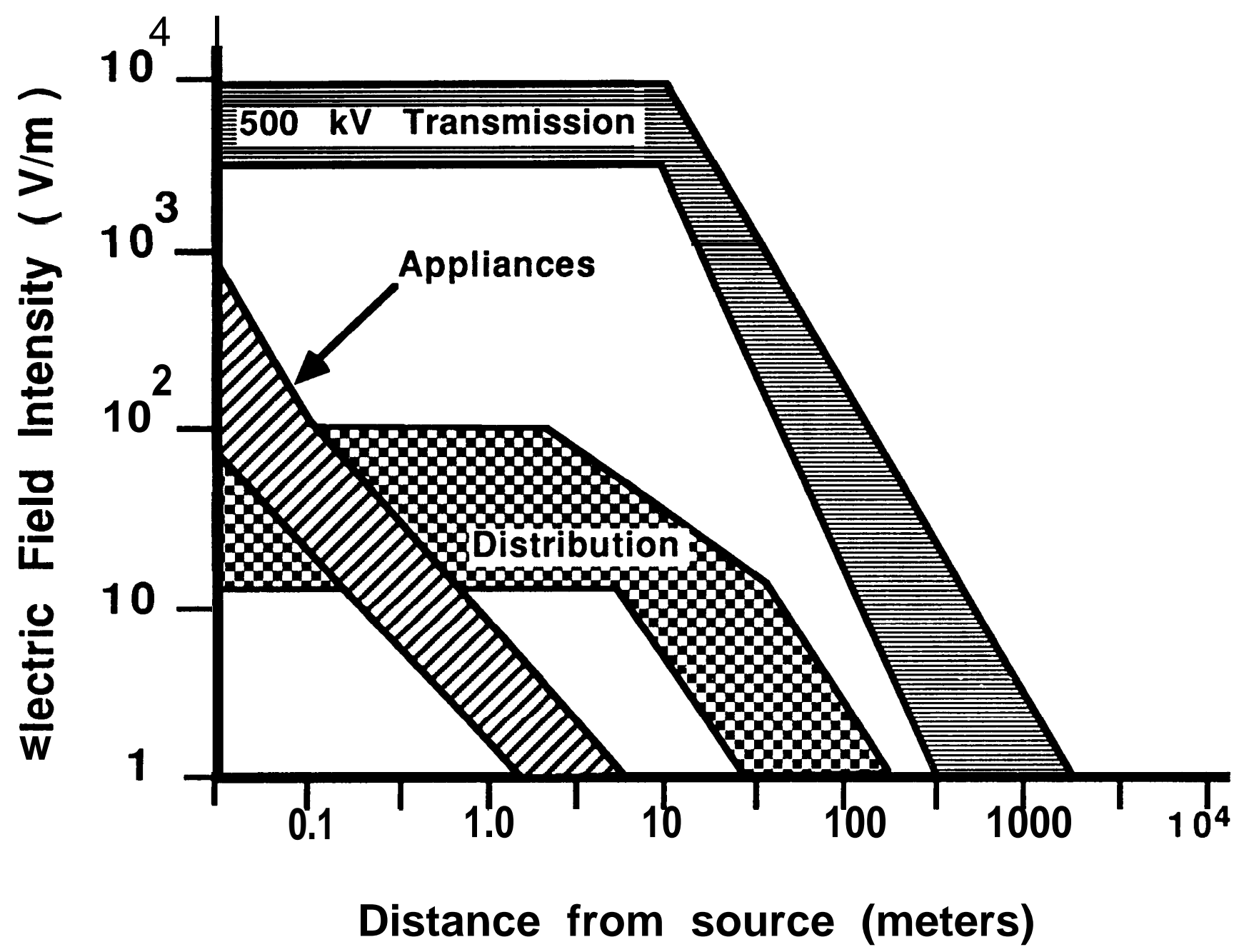

Figure 2-5: Illustration of how the electric field intensity at ground level changes with horizontal distance from three common sources of power-frequency electric fields. The bands represent variation across individual sources in each group. Adapted from [Florig 87a]. 


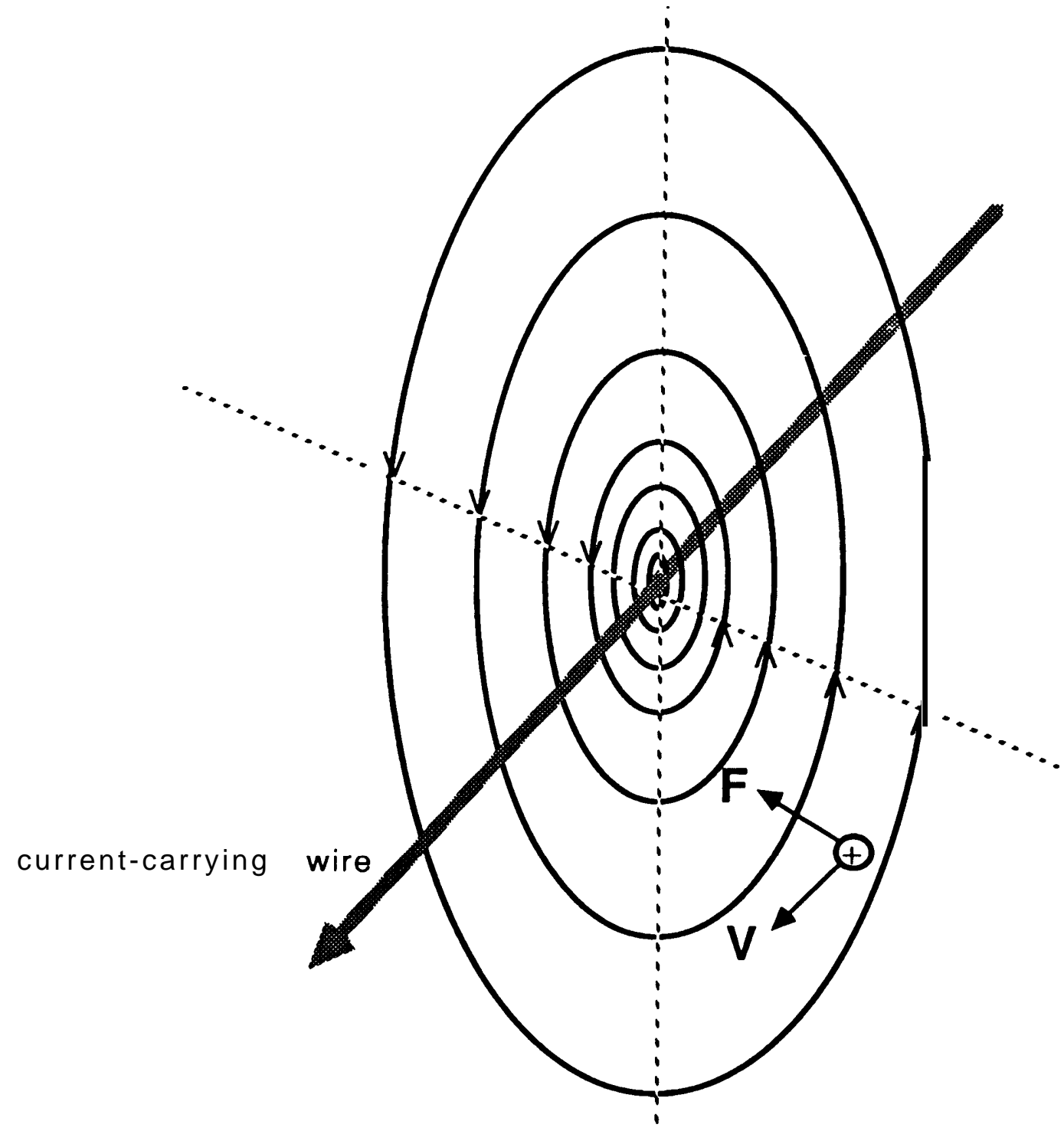

Figure 2-6: The magnetic field of a long straight wire produces a force, F, on a positively charged particle that is moving nearby. The strength of the field is proportional to the spacing between the lines (closer spacing means stronger field). The direction of the magnetic force on a charged particle moving in the field is perpendicular to both the field lines and the particle's direction of motion, $\mathrm{V}$. 

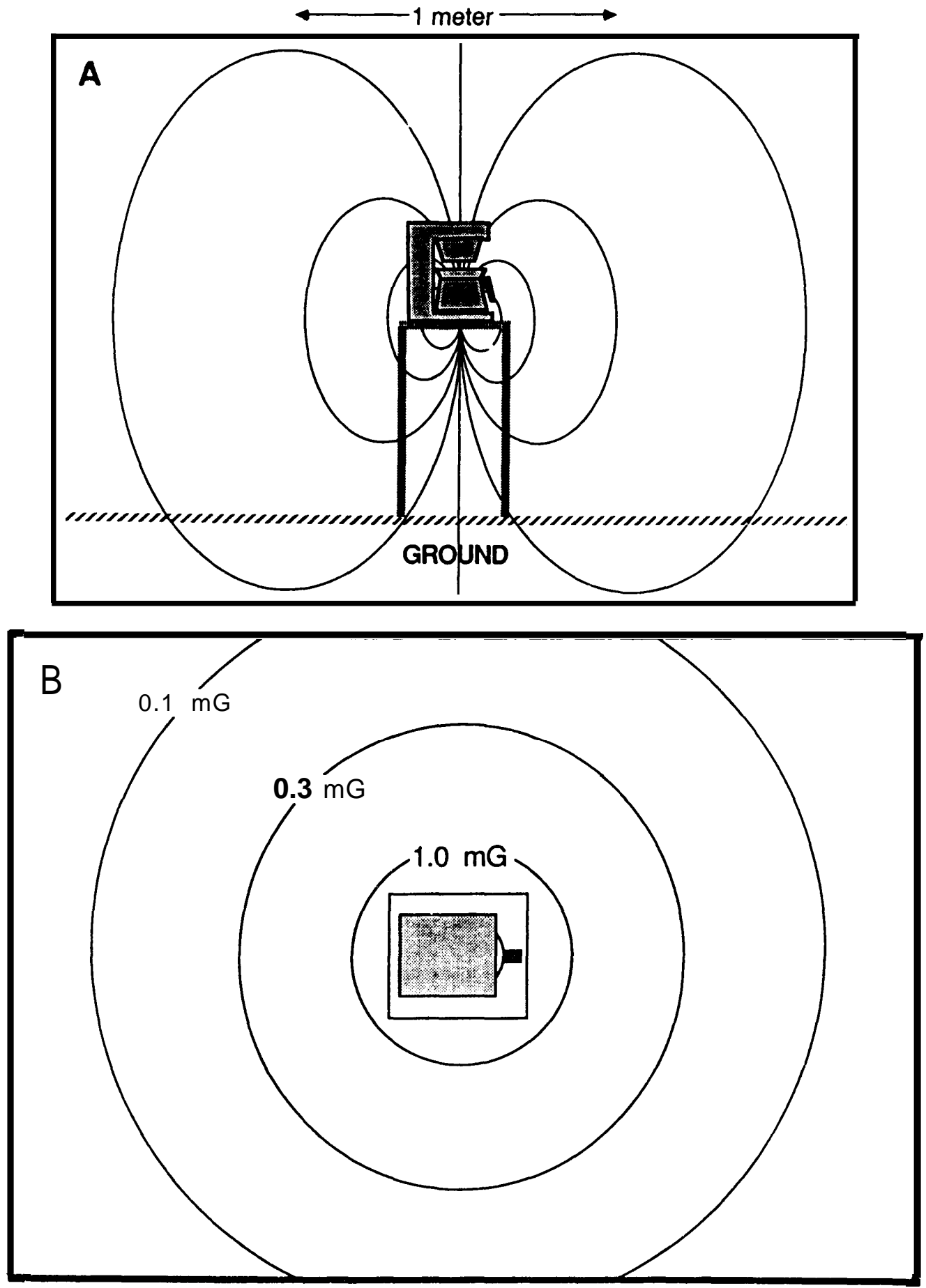

Figure 2-7: Time averaged (root mean square) magnetic field of a coffee maker (A) and top view (B) showing lines of equal flux density. Adapted from [Gauger 85]. 


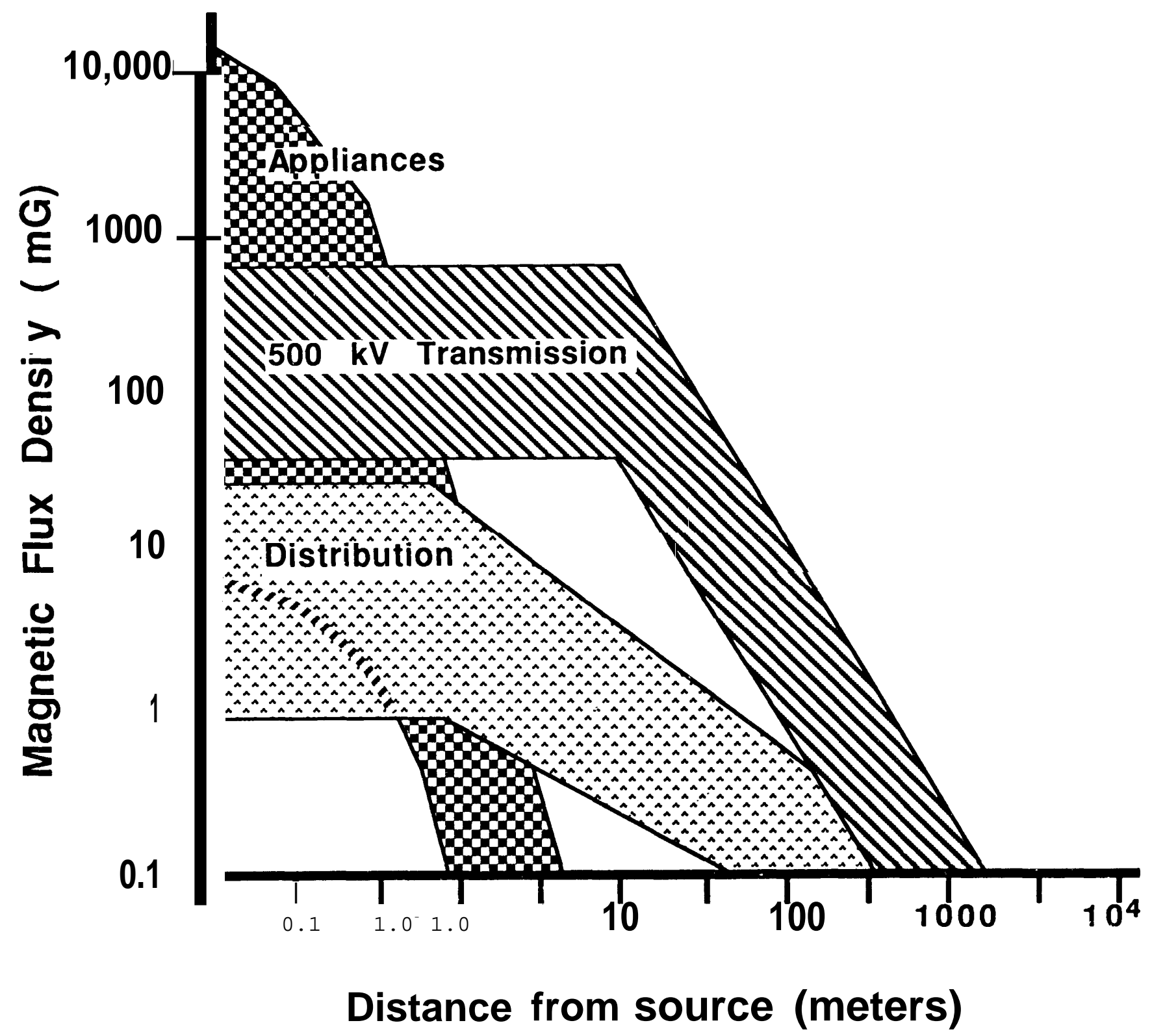

Figure 2-8: Illustration of how the magnetic field intensity at ground level changes with horizontal distance from three common sources of power-frequency magnetic fields. The bands represent variation across individual sources in each group. Adapted from [Florig 87a]. 
Table 2-1: Summary of published references which contain data on power frequency fields associated with various sources.

\begin{tabular}{|c|c|c|c|}
\hline Study & $\begin{array}{l}\text { Field: Electric (E) } \\
\text { Magnetic }(M)\end{array}$ & $\begin{array}{l}\text { Measured (m) } \\
\text { Computed (c) }\end{array}$ & Sources \\
\hline $\begin{array}{l}\text { [Bowman 88] } \\
\text { [Caola 83] } \\
\text { [Chartier 85] } \\
\text { [Deadman 88] } \\
\text { [Deno 78] } \\
\text { [Deno 82] } \\
\text { [Deno 87a] } \\
\text { [Deno 87b] } \\
\text { [Enk 84] } \\
\text { [Gauger 85] } \\
\text { [Florig 86] } \\
\text { [Florig 87b] } \\
\text { [Harvey 87] } \\
\text { [Heroux 87] } \\
\text { [IEEE 88] } \\
\text { [Jacobs 84] } \\
\text { [Kaune 87] } \\
\text { [Krause 85] } \\
\text { [Lovsund80 80] } \\
\text { [Male 871 } \\
\text { [Miller 74] } \\
\text { [Norris 871 } \\
\text { [Savitz 87a] } \\
\text { [Sendaula 84] } \\
\text { [-- 85] } \\
\text { [Silva 88] } \\
\text { [Stuchly 83] } \\
\text { [Tell 83] } \\
\text { [Tomenius 86] } \\
\text { [Valentino 72] } \\
\text { [Wertheimer 79, Wertheimer 82] }\end{array}$ & $\begin{array}{l}E, M \\
E \\
E \\
E, M \\
E, M \\
E, M \\
E, M \\
E, M \\
E, M \\
M \\
E \\
E \\
E, M \\
E, M \\
M \\
E \\
E, M \\
M \\
M \\
E, M \\
E, M \\
M \\
E, M \\
E, M \\
E \\
M \\
M \\
M \\
M \\
E, M \\
M\end{array}$ & $\begin{array}{l}\mathrm{m} \\
\mathrm{m} \\
\mathrm{m} \\
\mathrm{m} \\
\mathrm{c}, \mathrm{m} \\
\mathrm{c}, \mathrm{m} \\
\mathrm{c} \\
\mathrm{m} \\
\mathrm{m} \\
\mathrm{m} \\
\mathrm{c}, \mathrm{m} \\
\mathrm{c} \\
\mathrm{m} \\
\mathrm{m} \\
\mathrm{c}, \mathrm{m} \\
\mathrm{c}, \mathrm{m} \\
\mathrm{m} \\
\mathrm{m} \\
\mathrm{m} \\
\mathrm{c}, \mathrm{m} \\
\mathrm{m} \\
\mathrm{c}, \mathrm{m} \\
\mathrm{c}, \mathrm{m} \\
\mathrm{m} \\
\mathrm{m} \\
\mathrm{m} \\
\mathrm{m} \\
\mathrm{m} \\
\mathrm{m} \\
\mathrm{m} \\
\mathrm{m}\end{array}$ & $\begin{array}{l}\text { I,W } \\
\text { T } \\
\text { W } \\
\text { I,W } \\
\text { A, T } \\
\text { A, T } \\
\text { T } \\
\text { 1,0 } \\
\text { 1,0 } \\
\text { A } \\
\text { A, B, T } \\
\text { B } \\
\text { 1, T } \\
\text { D } \\
\text { D, T } \\
\text { T } \\
\text { I } \\
\text { I } \\
\text { w } \\
\text { 1, W } \\
\text { A, 1,0, T } \\
\text { A, B, D } \\
\text { D, I } \\
\text { T } \\
\text { 1,T } \\
\text { I } \\
\text { A } \\
\text { I } \\
\text { D } \\
\text { A, 1, T } \\
\text { A, D }\end{array}$ \\
\hline
\end{tabular}

Key to source column:

$T$ - transmission lines $B$ - electric blankets

D - distribution lines I - ambient indoor

A - appliances $\quad 0$ - ambient outdoor

W - work environment 
near appliances (particularly those with small motors or transformers such as hairdryers and fluorescent light fixtures). Because appliance fields fall off rapidly with distance and since people generally spend only brief amounts of time very close to appliances (with the exception of electric blankets and a few other appliances), appliances are usually not dominant contributors to time-averaged magnetic field exposure. However, since it is not known what aspect of the field, if any, is biologically important, care must be taken in making inferences about "exposure" from this fact.

Magnetic fields from wall wiring can be quite small because the field created by the current in the "hot" side of the line is canceled by the field created by the equal and opposite current in the parallel "neutral" (or ground) wire. This cancellation is greatest when the hot and neutral conductors are close together as they are in ROMEX cable or when both conductors are run through the same conduit. Many older homes have 'knob and tube*' wiring in which the hot and neutral conductors are separated by many inches. Wall wiring of this type can make significant contributions to the average magnetic field in homes.

Ground currents arise because the neutral (or grounded) wires of distribution lines are usually physically connected to the earth at many points along the line. These connections are made either through metal rods driven into the ground or by direct connection to water lines. Connections to earth are generally made at every distribution transformer and at every service drop (the point where electric lines enter the home). These ground connections provide alternate paths for distribution currents to return to local transformers or substations. This leads to power-frequency currents in water and gas plumbing. Because ground currents are not balanced by equal and opposite currents in parallel conductors, the magnetic fields that they produce can contribute substantially to the overall magnetic field in homes.

Barnes and colleagues found that houses in the Denver area were often close enough to overhead or underground distribution lines that the magnetic fields produced by the lines could account for a large fraction of the fields measured in the homes [Barnes 871. Their estimates of the contributions of appliances, house wiring, ground currents, and distribution lines to magnetic fields in houses is shown in Table 2-2. Again, because it is not clear what, if any, aspect of the field is biologically important, care should be taken in making inferences about "exposure" from these numbers.

Table 2-2: Sources of $60 \mathrm{~Hz}$ magnetic fields in residences. Adapted from [Barnes 87]

\begin{tabular}{ll} 
Source & Magnetic Flux Density \\
\hline Appliances & $6 \mathrm{mG}$ to $25 \mathrm{G}$ \\
House wiring & $.01 \mathrm{mG}$ to $10 \mathrm{mG}$ \\
Ground currents & up to $5 \mathrm{mG}$ \\
Distribution lines & $.01 \mathrm{mG}$ to $10 \mathrm{mG}$
\end{tabular}




\subsubsection{Shielding of Fields}

Trees, tall fences, buildings, and most other large structures provide shielding from electric fields. The presence of these structures can, therefore, have a significant effect on the electric fields to which people are exposed. Houses, for instance, attenuate electric fields from nearby power lines by roughly $90 \%$ [Florig 86]. Shielding by other objects can be equally great [Deno 87a]. Magnetic fields are shielded only by structures containing large amounts of ferrous or other special metals. Houses, trees, and most other objects, therefore, do not provide appreciable shielding of magnetic fields.

\subsection{Electric and Magnetic Induction}

The human body contains free electric charges (largely in ion-rich fluids such as blood and lymph) that move in response to forces exerted by charges on and currents flowing in nearby power lines and appliances. The processes that produce these body currents are called electric and magnetic induction.

In electric induction, charges on a power line or appliance attract or repel free charges within the body. Since body fluids are good conductors of electricity, charges in the body move to its surface under the influence of this electric force. For example, a positively charged overhead transmission line induces negative charges to flow to the surfaces on the upper part of the body as shown in Figure 2-9. Since the charge on power lines alternates from positive to negative many times each second, the charges induced on the body surface alternate also. Negative charges induced on the upper part of the body one instant flow into the lower part of the body the next instant. Thus, power-frequency electric fields induce currents in the body as well as charges on its surface. A number of investigators have studied the surface charges and internal currents that are induced by power-frequency electric fields in both people and animals. A review of the electric induction literature has been written by Kaune [Kaune 85].

Magnetic fields are intimately related to electric fields. This relationship was first fully described by physicist James Clerk Maxwell in the nineteenth century. Among other things, Maxwell showed that changing magnetic fields produce electric fields. Because power-frequency circuits contain alternating currents, they produce changing magnetic fields. The electric fields produced by these changing magnetic fields exert forces on electrical charges contained within the body. This process is called magnetic induction.

Magnetically-induced currents flow in loops which is why they are sometimes referred to as "eddy" currents. The nature of magnetic induction is such that currents induced in the body by magnetic fields are greatest near the periphery of the body and smallest at the center of the body (see Figure 2-10). Because magnetic fields have only recently become a human health concern, data on the detailed distribution of magnetically-induced currents in humans and animals is quite sparse compared to the information available on electric induction. Studies of magnetic induction include the theoretical work of Spiegel [Spiegel 76, Spiegel 771 and Kaune [Kaune 86] and measurements by Guy and colleagues [Guy 76]. The lack of detailed data on magnetic induction makes it difficult to compare the body currents induced by the electric and magnetic fields of any given source.

The magnitude of surface charge and internal body currents that are induced by any given source of power-frequency fields depends on many factors. These include the magnitude of the charges and currents in the source, the distance of the body from the source, the presence of other objects that might shield or concentrate the field, and body posture, shape, and orientation. For this reason the surface charges and currents which a given field induces are very different for different animals. 


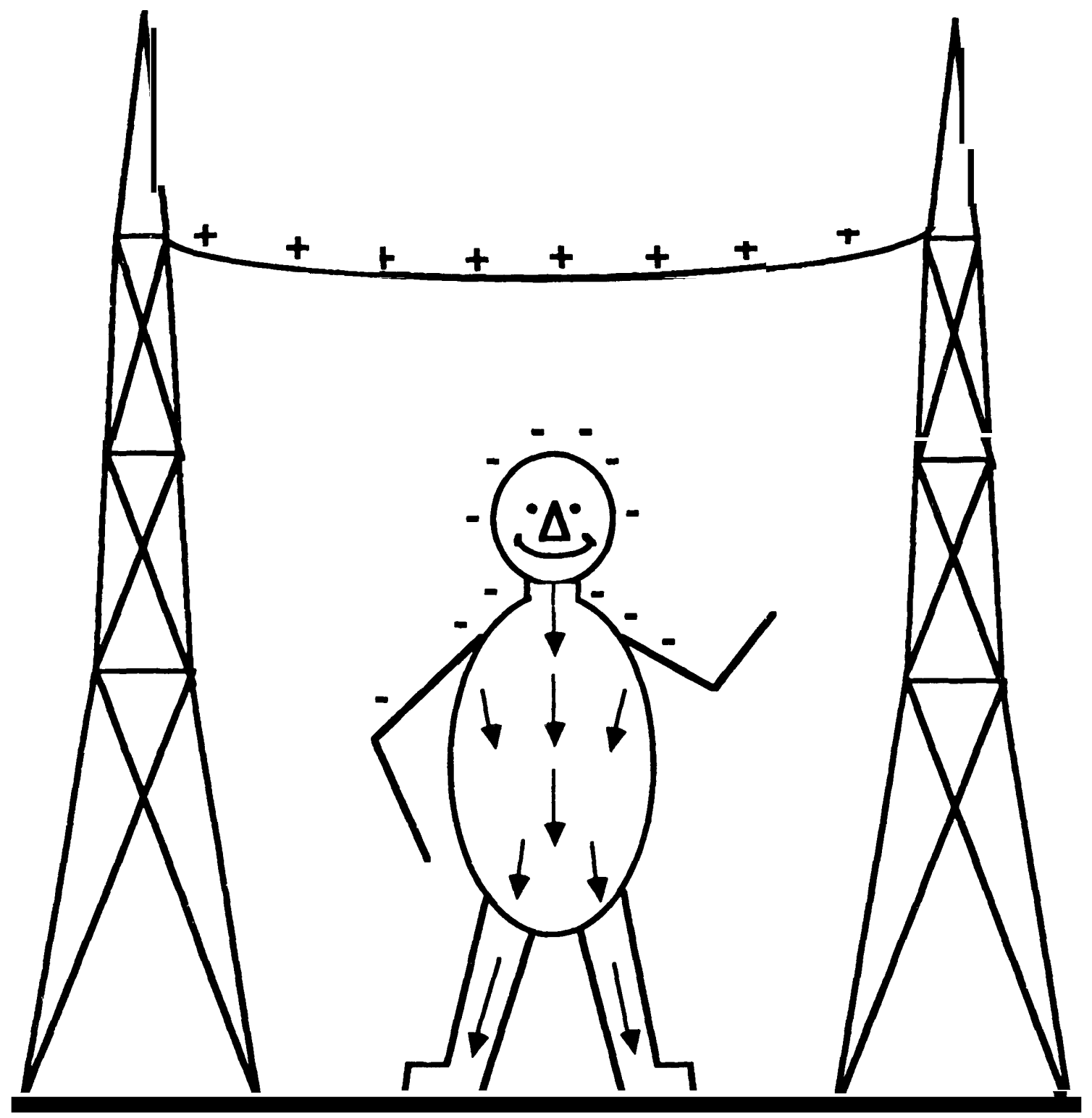

Figure 2-9: A schematic representation of the surface charges and internal currents that are electrically induced by the charges on an overhead power line in a person under the line whose feet are well-grounded. The total current induced to flow from each foot to ground is about 8 microamps per $\mathrm{kV} / \mathrm{m}$ of applied field (1 microamp is 1 millionth of an ampere). The density of electrically-induced current is the amount of current that passes through a body crosssection perpendicular to the direction of current flow. The current density induced by a 1 $\mathrm{kV} / \mathrm{m}$ vertical electric field is about 30 nanoamps per square centimeter averaged over the entire volume of the body. One nanoamp is 1 billionth of an ampere. 


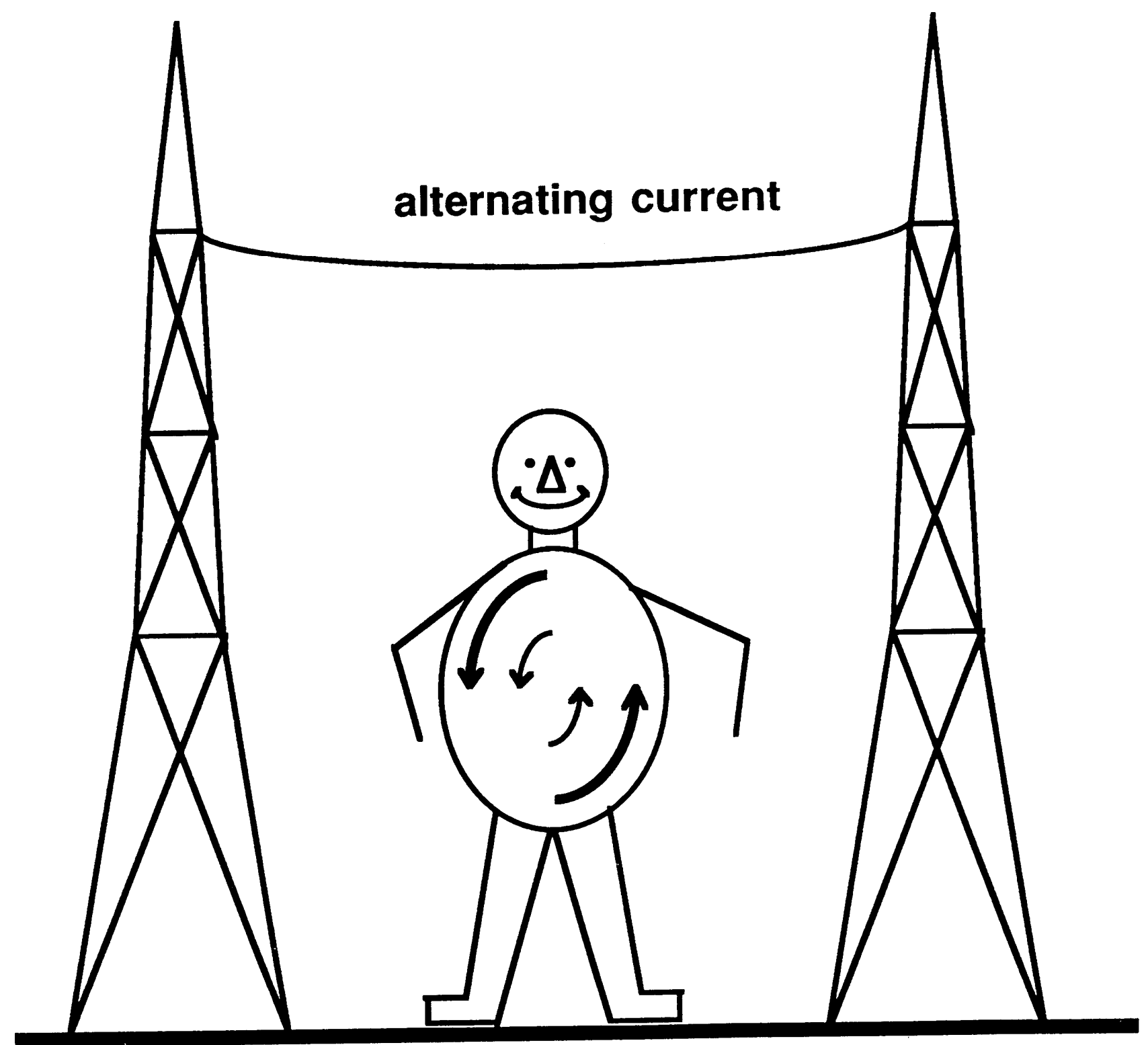

Figure 2-10: A schematic representation of the pattern of currents induced in the body of a person standing under a transmission line by the alternating magnetic field set up by the current flowing in that line. A $60 \mathrm{~Hz}$ magnetic field with a flux density of one gauss will induce currents in the periphery of the body with a current density of about 100 nano Amps per square centimeter. The current density at the center of the body is zero. 


\subsection{Contact Currents}

Besides direct electric and magnetic induction, another source of power-frequency exposure is contact currents. Contact currents are the currents that flow into the body when physical contact is made between the body and a conducting object carrying an induced voltage. Examples of contact current situations include contacts with vehicles parked under transmission lines and contacts with the metal parts of appliances such as the handle of a refrigerator. Contact currents are important because they often produce high current densities in the tissue near the point of contact. Although contact currents result in exposure to some of the most intense currents, they are also among the briefest, usually lasting only as long as it takes to open the door of the car or refrigerator.

If a person touches a vehicle parked under an overhead power line, the body provides a path to the ground through which charge induced on the vehicle by the electric field of the power line can flow. The magnitude of contact current depends on a number of factors including the local field intensity, the size and shape of the contacted object, and how well grounded the contacted object and the person are. The largest contact currents are drawn by well-grounded persons who touch large metal objects that are well-insulated from ground. The most common contact currents are imperceptibly small (less than 2 milliamps). Under the right circumstances, however, contact currents can be annoying or even painful. To protect the public from life-threatening contact currents, the American National Standards Institute (ANSI) has recommended that overhead lines be designed so that contact currents from even very large vehicles do not exceed 5 milliamps [ANSI 77]. Because 5 milliamps delivers a very unpleasant shock to an adult and is above the "let-go" threshold for some children, there is some concern that the ANSI limit is not conservative enough. The let-go threshold is the current above which a person loses voluntary muscle control and cannot "let go" of a gripped contact.

Contact currents associated with appliances are also usually imperceptibly small. An upper limit on appliance contact current is given by the appliance short-circuit current, which is the contact current that would flow into a person who has wet hands and is well-grounded. Measurements indicate that typical appliance short-circuit currents lie in the range of 1-100 $\mu \mathrm{A}(1 \mu \mathrm{A}=1$ millionth of an Amp) [Florig 86]. Short-circuit currents for new appliances are currently limited by ANSI standard to $.5 \mathrm{~mA}(1 \mathrm{~mA}=1$ thousandth of an Amp) for portable appliances and to $.75 \mathrm{~mA}$ for stationary units. It is apparent from available data that appliance manufacturers have no trouble meeting these requirements. While contact currents typically flow for only short periods of times (for example, while your hand is on the refrigerator door), the currents involved and the associated fields in the body can be quite high compared to those induced by the fields of overhead power lines.

\subsection{Measuring "Dose"}

Although it is possible to measure or compute the fields and induced currents to which people are exposed, scientists do not know which, if any, of these quantities might be related to human health impact. Scientists do not know whether we should be concerned with the strength of the field, the change in field strength over time, the currents induced in the body, or some other variable. Uncertainty is common when dealing with environmental risks, but the case of electromagnetic fields differs from that of most environmental agents. For most known of potential hazards, such as chemicals, one can safely assume that if some of the agent is bad, more of it is worse. Unfortunately, as we explain in later sections, much of the biological experimental evidence about power-frequency fields suggests that the more-is-worse assumption cannot always be justified. 
The problem involves the definition of dose: identifying which, if any, aspect of the field can affect health. With a chemical, dose is typically defined as the amount that gets into people, or, if the body is able to metabolize or get rid of the chemical, the rate at which the chemical enters the body. Although some human epidemiological studies of the bioeffects of power-frequency fields have suggested a dose measure that is proportional to the long-term average of peoples' magnetic field exposure, other studies have suggested very different measures of dose. Examples, described in detail in Section 3 include:

. Frequency and intensity "windows": Experiments in which biological effects are seen only in specific narrow ranges of field intensity and frequency, [Bawin 76, Blackman 85a].

Time thresholds: Experiments in which field effects are observed only after several weeks of exposure [Wilson 81, Wilson 83],

Transient responses: Studies in which field exposure induces a biological effect for only a short time after a change in the exposure [Byus 86].

- Field threshold: Effects that appear only when the field strength exceeds some threshold value [Liboff 84].

Although each of these experiments involves a different protocol and biological system, together they suggest that one may be unjustified in making the simple assumption that dose is proportional to field strength or to time spent in the field.

\subsection{Comparing Human Exposures from Different Sources}

As explained in Section 2.5, there is no accepted measure of the biological effectiveness of powerfrequency fields. Comparisons of peoples' exposures to different sources of power-frequency fields, therefore, cannot be made on the basis of relative contribution to effective dose. Given the current state of the health effects science, comparisons between sources can be based only on those physical quantities that are amenable to measurement or theoretical estimates. These include electrical quantities such as induced surface charge and internal currents as well as quantities that describe exposure duration, how often an exposure occurs, and the numbers of people exposed. Although these quantities may not relate in any simple way to the possible public health impact of a given source, one can use them to get some idea of how similar or different peoples' exposures to various sources are. In the next few pages, peoples' exposures to power line fields and appliances are compared in six ways using 1) bodyaverage current density, 2) body-average surface electric field, 3) body-average magnetic field, 4) peak current density, 5) typical exposure duration, and 6) the fraction of the total population exposed. Eight different exposure situations, each involving electric and magnetic fields from just one type of source, are chosen for comparison. These are:

1. Induction from transmission line fields in a person standing on the right-of-way (RoW) of a $500 \mathrm{kV}$ line.

2. Induction from transmission line fields in a person inside a house located between the edge of the RoW and 100 meters from the centerline of a $500 \mathrm{kV}$ line.

3. Contact current from an automobile parked within $100 \mathrm{~m}$ of the centerline of a $500 \mathrm{kV}$ line.

4. Contact current from a typical appliance (e.g. toaster, refrigerator)

5. Induction from an electric blanket.

6. Induction from an electric shaver.

7. Induction from indoor background fields that arise from appliances plumbing, and wall wiring. 
8. Induction from distribution line fields in a person standing beneath a $35 \mathrm{kV}$ distribution line.

Exposure comparisons for these eight situations are presented in Figures 2-11 and 2-12. Exposure estimates are calculated from information in many of the sources referenced in sections 2.2, 2.3, and 2.4 as well as other data [Florig 87b, ICRP 75, Juster 79]. The range of values indicated for each of the entries in these figures represents both uncertainty in the various factors needed to estimate the dose (dosimetric factors) and variability across the exposed population. Note that the scale in each figure is logarithmic.

The most important message that these figures convey is that a ranking of different exposure situations along one dimension can look quite different from the ranking along another dimension. Exposures in transmission line rights-of-way, for instance, score high on the intensity-related dimensions but low on the duration and prevalence dimensions.

\subsection{Sources of Exposure at Non-Power Frequencies}

Power-frequency fields are only one component of the non-ionizing electric and magnetic fields that people regularly encounter. Electric and magnetic fields at higher frequencies are produced by a wide variety of modern devices such as stereo headphones ( about $1 \mathrm{kHz}$ ), TV sets (about $20 \mathrm{kHz}$ ), AM radio transmitters (about $1 \mathrm{MHz}$ ), CB radios (about $30 \mathrm{MHz}$ ), FM radio and TV transmitters (about $100 \mathrm{MHz}$ ) and microwave ovens (about $2 \mathrm{GHz})^{2}$.

Scientists do not know whether fields at these higher frequencies are either more or less biologically effective than power-frequency fields. There is evidence that VLF-modulated high frequency fields can produce effects similar to those of ELF fields. Many of these higher-frequency sources induce more intense currents in the body than are induced by most power-frequency sources. For example, the $20 \mathrm{kHz}$ electric field at 1 meter from a television set, induces body currents that are comparable in magnitude to those induced by a power-frequency electric field of $3 \mathrm{kV} / \mathrm{m}$.

${ }_{1} \mathrm{kHz}=1$ thousand cycles per second, $1 \mathrm{MHz} .1$ million cycles per second, $1 \mathrm{GHz}=1$ billion cycles Per second. 
A

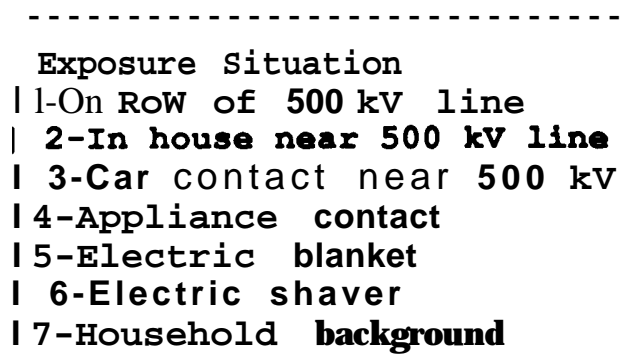

8-Beneath distribution line

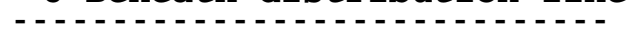

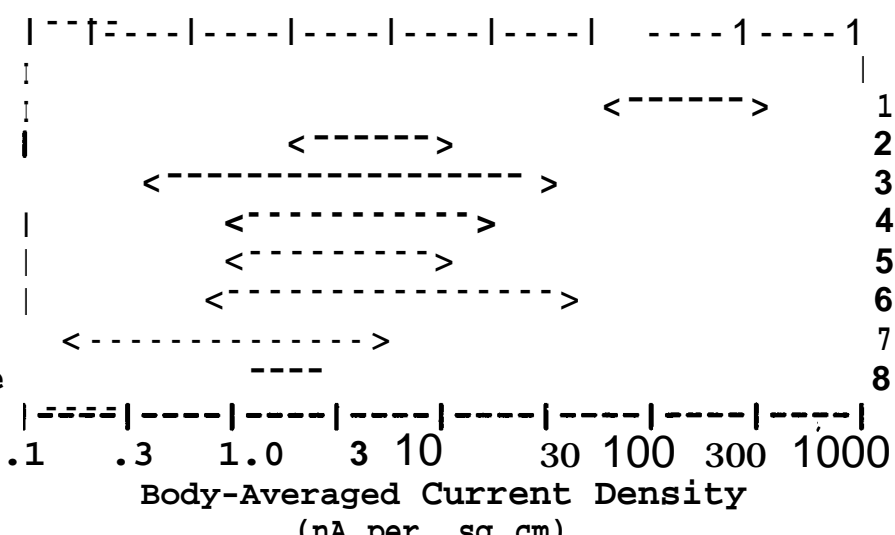

(nA per sq cm)

B

[Exposure Situation
1-On RoW of $500 \mathrm{kV}$ line
2-In house near $500 \mathrm{kV}$ line
3-Car contact near $500 \mathrm{kV}$
4-Appliance contact

5-Electric blanket

6-Electric shaver

7-Household background

8-Beneath distribution line

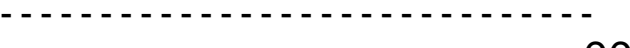

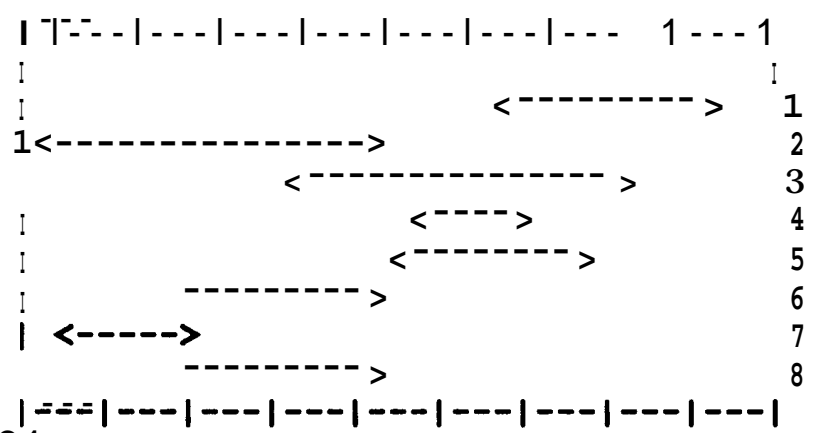

$\begin{array}{llllllllll}.001 & 003 & .01 & .03 & .1 & .3 & 1 & 3 & 10 & 30\end{array}$

Body-Averaged Surface Field

$(\mathrm{kV} / \mathrm{m})$

C

Exposure Situation

$1-$ On RoW of $500 \mathrm{kV}$ line

2-In house near $500 \mathrm{kV}$ line

3-Car contact near $500 \mathrm{kV}$

4-Appliance contact

5-Electric blanket

6-Electric shaver

7-Household background

8 -Beneath distribution line

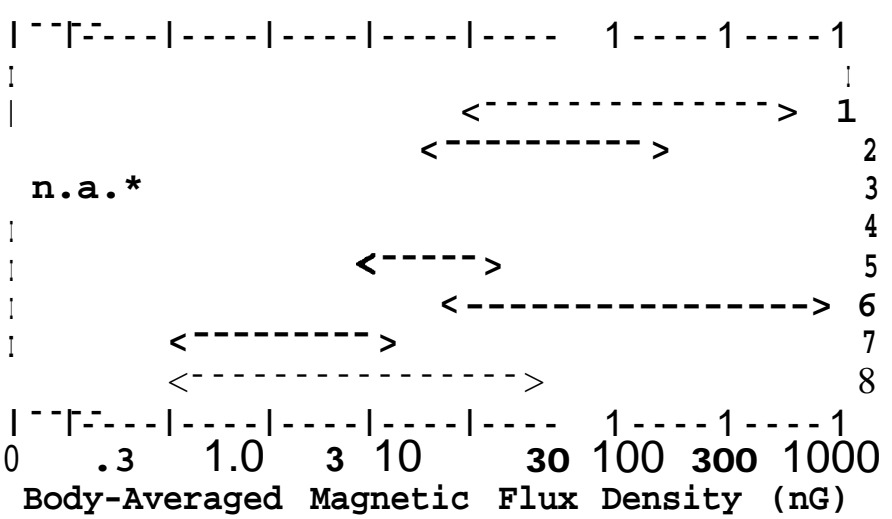

* contact currents themselves produce negligible magnetic field. Of course the magnetic fields from the nearby $500 \mathrm{kV}$ line would be comparable to those of exposure situations C1 and C2.

Figure 2-11: Three different exposure measures applied to 8 exposure situations. Exposure measures are A) the density of electrically and magnetically-induced currents averaged over the body, B) the induced electric field averaged over the body surface, and C) the average magnetic field within the body. Ranges represent the span of typical values. 
D

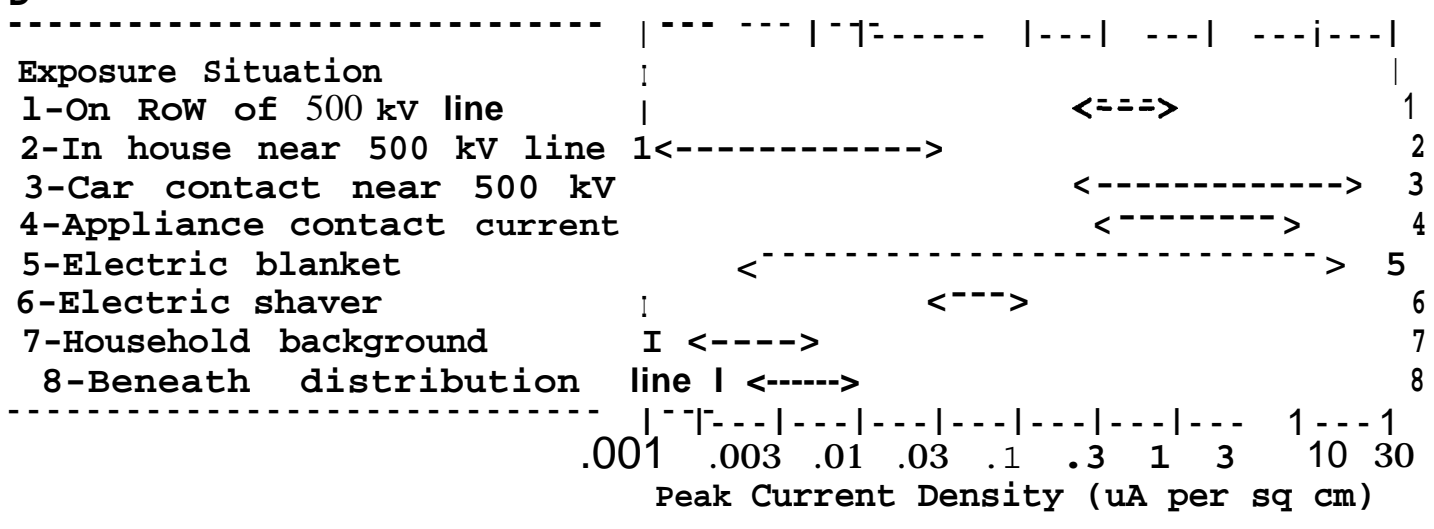

E

Exposure Situation

I-On Row of $500 \mathrm{kV}$ line

2-In house near $500 \mathrm{kV}$ line

3-Car contact near $500 \mathrm{kV}$ ]

4-Appliance contact

5-Electric blanket

6-Electric shaver

7-Household background

8-Beneath distribution line

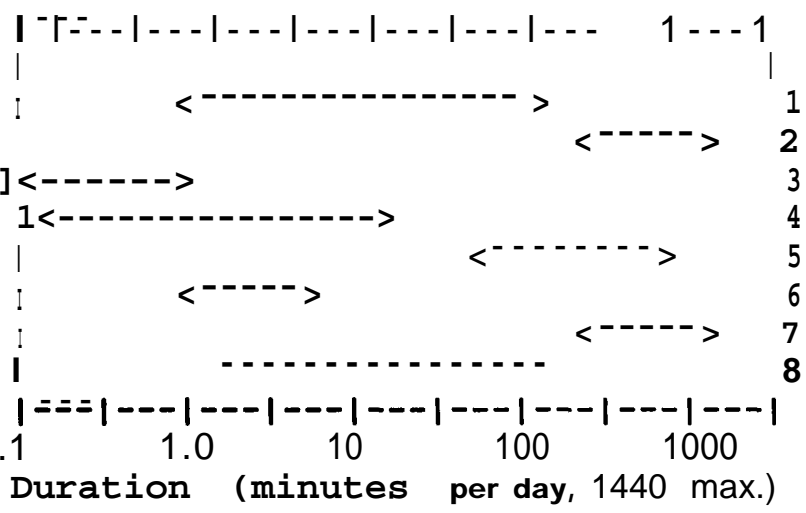

$\mathbf{F}$

Exposure Situation

l-On RoW of $500 \mathrm{kV}$ line

2-In house near $500 \mathrm{kV}$ line

3-Car contact near $500 \mathrm{kV}$

4-Appliance contact

5-Electric blanket

6-Electric shaver

7-Household background

8-Beneath distribution line

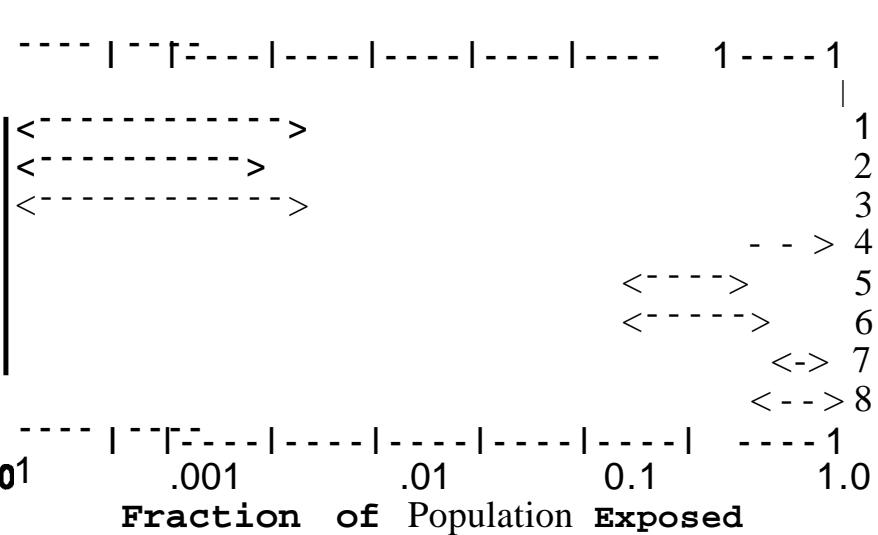

Figure 2-12: Three more exposure measures applied to 8 exposure situations. Exposure measures are D) peak electrically or magnetically induced current density anywhere in the body, E) the duration of the field encounter and $F$ ) the fraction of the population that regularly encounter the exposure situation. Ranges represent the span of typical values. 
The previous sections have described what is meant by fields, and how exposure to fields is measured in actual and laboratory settings. The understanding of what the effects of this exposure may be comes from three kinds of studies.

1. Laboratory experiments using animal or human tissues or cell cultures exposed to fields. These experiments are termed 'in vitro" (in glass) experiments.

2. Laboratory and field experiments using live animals and people exposed to fields. These experiments are termed "in vivo" (in a live state) experiments.

3. Epidemological studies involving human populations exposed to fields at work (occupational studies) or at home (residential studies).

The following sections describe these in order.

\section{Cellular Level Experiments}

Until the mid-1970's few conjectured that there could be any effect on a biological system from electric or magnetic fields of the strengths usually present in the environment. This was in part due to the fact that these fields can transfer only minute amounts of energy to the cell, and hence can not disrupt chemical bonds in the cell as ionizing radiation can. There is not even enough energy in $60 \mathrm{~Hz}$ fields to heat the cell to any significant extent as microwave or radiofrequency radiation does.

A considerable body of evidence has emerged that points to the cell membrane (the membrane enveloping the cell) as the primary site of interaction between ELF fields and the cell [Adey 86]. In addition to sewing as the boundary and maintaining the structural integrity of the cell, the cell membrane is responsible for some of the critical functions of the cell such as controlling the flows of material and energy signals into the cell and transmitting information arriving at its surface to the interior of the cell so that appropriate life processes can take place. It is a highly selective filter that maintains an unequal concentration of ions (charged atoms) on either side and allows nutrients to enter and waste products to leave the cell. This is made possible by very specialized components of the cell membrane.

Unequal concentrations of ions are used by the cell for transmitting external signals to the interior; and for allowing or preventing the entry of selected molecules and ions into the cell. The most important ions are potassium $(\mathrm{K}+)$, sodium $\left(\mathrm{Na}^{+}\right)$, chlorine $(\mathrm{Cl}-)$, hydrogen $(\mathrm{H}+)$, and calcium $\left(\mathrm{Ca}^{+2}\right)$. The actual entry of many molecules and ions occur through channels in the cell called "ion channels". These close or open in response to the ion concentrations and thus regulate the flows. There are also some enzymes that are attached to the membrane. These "membrane-bound" enzymes take part in the synthesis of molecules as well as in controlling initial actions of external molecules such as drugs.

ELF field experiments on the cellular level have concentrated on examining how some of the specific processes governed by the membrane change as a result of exposure to the ELF fields. When reading the results, it is important to understand that even when an effect is observed at the cellular level, it is still hard to extrapolate what, if anything, that effect implies for the organism as a whole.

In this section, experimental results on effects of ELF field exposure on cell cultures are discussed under six classes:

1. modulation of ion flows [Bawin 76, Bawin 75, Blackman 82, Blackman 85a];

2. interference with DNA synthesis and RNA transcription [Liboff 84, Goodman 86, Goodman 87] 
3. interaction with the cell response to different hormones and enzymes including those that are involved in cell growth processes and stress responses [Luben 82, Lymangrover 83, Lymangrover 87];

4. interaction with the cell response to chemical neurotransmitters [Vasquez 86];

5. interaction with the immune response of cells [Lyle 83]; and

6. interaction with cancerous cells [Cain 86, Winters 86]

The observed effects have certain peculiarities. Some effects described below occur at some frequency and intensity values but not at others. Some effects depend upon the duration of the exposure. Some effects persist only for a brief period of time after the exposure is discontinued. These peculiarities make it difficult to extrapolate experience from the more familiar chemical and ionizing radiation toxicology to the realm of ELF exposure and effects.

Many of the studies described below have been carried out in single laboratories and, with a few exceptions, have not been replicated in other laboratories. Although a number of high quality experiments have been performed in the last decade, we still do not have enough robust, replicated results to build a coherent scientific or phenomenological picture of the spectrum of effects and we are far from having a good theoretical model. Generally, when the background science is clear and developed, experimental research on a new phenomenon proceeds by a series of steps usually referred to as "the scientific method". The first step in this method is to make a hypothesis (educated prediction) of the expected result. Experiments are then designed and conducted to "test the hypothesis". In the field of biological effects of ELF fields, there are not yet theories to make firm hypotheses and test them. Therefore, some of the experiments described in this paper are attempts to see if there are any effects at all. Other experiments examine if there are simple hypothetical connections between experimental observations and possible health effects. We are not at the stage where experiments can be designed to test hypotheses based on a coherent framework.

The most concerted of the studies on cellular level have been conducted by an interdisciplinary group of biologists, biochemists, physicians, physicists and psychologists at the Jerry L. Pettis Memorial Hospital at Loma Linda. This group has examined the various aspects of the interaction of ELF fields with the cell, paying particular attention to understanding the role of the cell membrane in the interaction.

Because of the rapidly evolving nature of this subject, a few of the results we discuss have been reported only in professional meetings and have not yet appeared in refereed journals. These distinctions are made clear in the references.

\subsection{Modulation of lon and Protein Flow across the Cell membrane}

The phenomenon most studied at the cellular level is the nonlinear pattern of calcium ion efflux from cells which results after exposure to $60 \mathrm{~Hz}$ fields. Before discussing these experiments it is useful to briefly review the role of calcium in the regulation of cellular processes.

The flow of calcium ions $\left(\mathrm{Ca}^{+2}\right)$ across the cell membrane in response to extracellular signals is an important means of transmitting signals from the outside to the interior of the cell. Calcium flow governs physiological processes such as muscle contraction, egg fertilization, and cell division. Most of the intracellular calcium (Ca present in the cell) is normally bound to molecules in the cell. Calcium is also 
present in the structure of the membrane itself, to be released in the event of an appropriate triggering signal.

When information in the form of electrical or chemical impulses arrives at the cell membrane, the membrane binding as well as permeability to calcium is altered and the subsequent transport of calcium across the membrane transfers the information signal to the interior of the cell. Because of this function, calcium is said to be a "second messenger".

Calcium acts in a multitude of ways in its capacity as a second messenger. For example, among the proteins on the surface of nerve cells are certain enzymes called calcium-dependent protein kinases which, when activated by the calcium changes, cause actions on other cell surface proteins that are important in cell adhesion during development and growth. Calcium signals also regulate processes such as muscle contractions including heartbeats, developmental processes such as egg maturation and ovulation and several others. The quantity as well as the rate of calcium ion transport are important in this regulation.

Unusual behavior of calcium efflux (or, outward flow) from cell membranes in brain tissue in vitro was the first clear, reproducible effect of ELF fields observed in biological tissue. Bawin and coworkers took the two halves of the brain of freshly killed chicks maintained in solutions to continue the natural cellular processes ("tissue preparation of chick cerebral hemisphere"). They exposed one half to an ELF field, keeping the other half unexposed to field. They then compared the calcium efflux from the two halves and found that the efflux was decreased in the exposed, compared to the unexposed half. This effect of decreased calcium efflux was noted to have frequency and amplitude "windows" around 6 and $16 \mathrm{~Hz}$ and at $10 \mathrm{~V} / \mathrm{m}$. That is, the effect occurred when the field value was $10 \mathrm{~V} / \mathrm{m}$ and the frequency $6 \mathrm{~Hz}$ or $16 \mathrm{~Hz}$. [Bawin 76].

In an independent set of experiments, Blackman and coworkers also observed a change in calcium efflux, although it was an increase rather than decrease, with a complex pattern of several "windows". The frequency ranges they examined were $1-30 \mathrm{~Hz}$ and $45-105 \mathrm{~Hz}$, and the intensity range, $1 \mathrm{Ho} 70 \mathrm{~V} / \mathrm{m}$. [Blackman 82, Blackman 85a, Blackman 85b]

Figure 3-1 summarizes the ELF frequency and intensity combinations found to cause changes in calcium-ion efflux from chick brain tissue in vitro. [Blackman 85a] For example, the figure shows that in this experiment, at $60 \mathrm{~Hz}$, six field intensity values were examined : 25, 30, 35, 40, 42.5 and $45 \mathrm{~V} / \mathrm{m}$. These voltage values to the peak-to-peak voltage (abbreviated as $\mathrm{V}_{\mathrm{pP}}$ ) of the field applied during the experiments. In all other experiments the field intensity is expressed in terms of root-mean-square (rms) voltage, as defined in Section 2.2.2. Figure 3-1 also shows the equivalent rms values. The effect appeared only at three of these values, 35,40 and $42.5 \mathrm{~V} / \mathrm{m}$. The lower values of 25 and $30 \mathrm{~V} / \mathrm{m}$ as well as the higher value of $45 \mathrm{~V} / \mathrm{m}$ showed no significant difference between the efflux from the exposed and the unexposed halves of the brain. That is, for the frequency of $60 \mathrm{~Hz}$, there is an intensity window between about $35 \mathrm{~V} / \mathrm{m}$ and $43 \mathrm{~V} / \mathrm{m}$. There is no effect observed immediately above or below this range of values. Similarly, if the intensity is kept at $42.5 \mathrm{~V} / \mathrm{m}$ and the effect explored at various frequencies, the effect is seen at several values $(15,45,60,75,90$ and $105 \mathrm{~V} / \mathrm{m})$ but not at values in between.

Further experiments by Blackman et al., showed that the position of frequency and amplitude windows was influenced by the strength and relative orientation of any static magnetic field superimposed on the AC field [Blackman $85 \mathrm{~b}$ ]. That is, the position of the windows depended on constant or static (not 


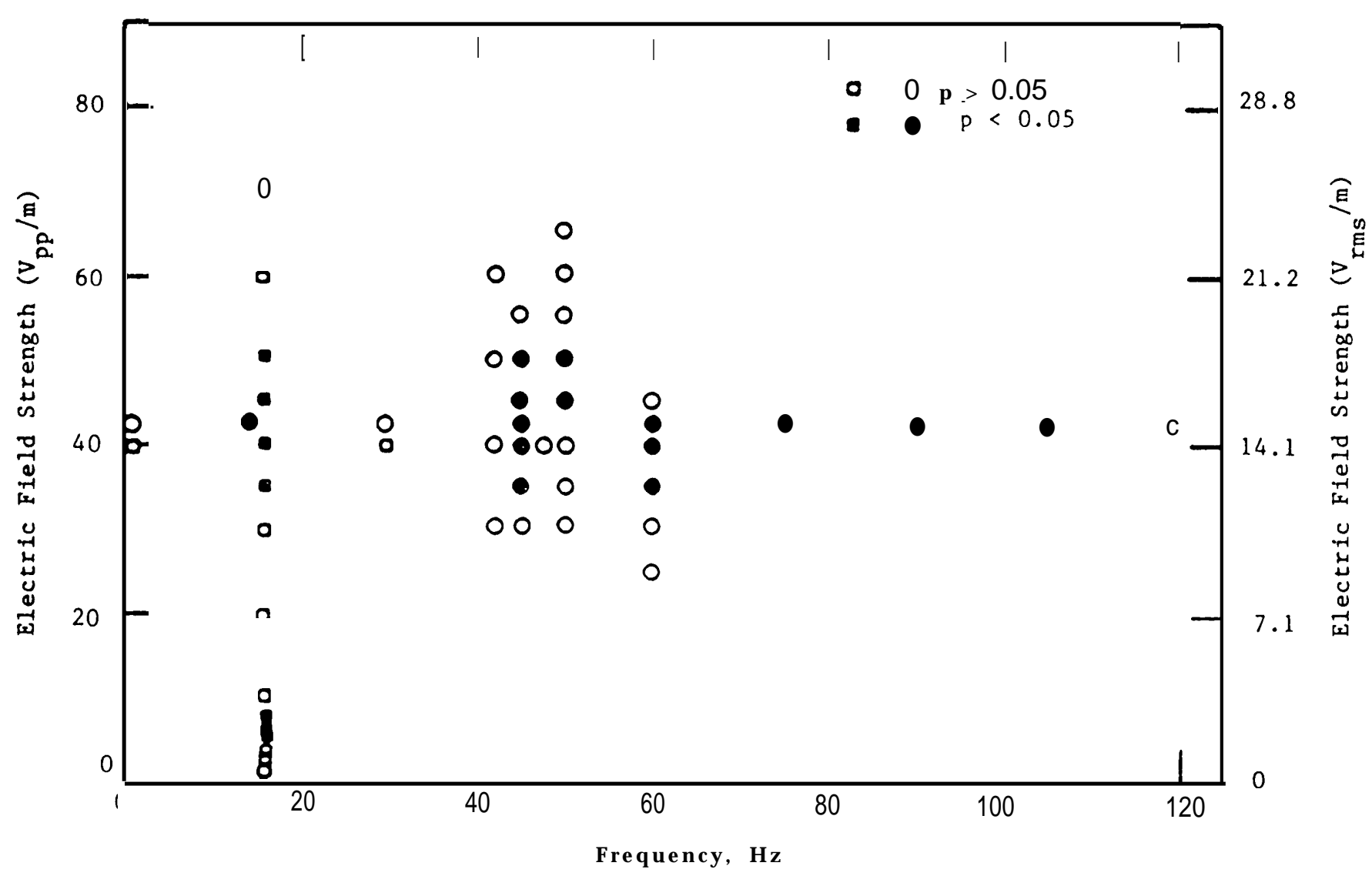

Figure 3-1: Frequency and intensity windows in the efflux of calcium from chick brain tissue : This figure shows the results of Blackman's experiments on calcium efflux in chick brain exposed to ELF electric fields.' The horizontal axis shows the frequency of the sinusoidal ELF exposure. The left hand vertical axis shows the peak-to peak electric field strength. The right hand vertical axis denotes the root-mean-square field strength (See section 2.2.I for an explanation). The efflux of calcium from one half of the brain exposed in vitro to ELF electric fields was found to be significantly higher than from the other half which was not exposed. The graph shows the values of frequency and field intensities for which there was an effect. Dark circles show the presence of an effect and the open circles show values which were examined, but no effect was found. Instead of showing an effect that increased or decreased with increasing or decreasing frequency or intensity, the effect appeared at certain values of frequency and intensity but not at others. For example, note that when the frequency of the applied field is $60 \mathrm{~Hz}$, an effect is seen at 3 values of field, at 35, 40 and $42.5 \mathrm{Vpp} / \mathrm{m}$. When the field is above or below this range, such as at $45 \mathrm{~V} / \mathrm{m}$ or at $30 \mathrm{Vpp} / \mathrm{m}$, there is no effect. So when the "applied frequency is $60 \mathrm{~Hz}$, there is an "intensity window" between about 35 and $42.5 \mathrm{Vpp} / \mathrm{m}$ at which there is an effect. Along similar lines, it can be seen that there are "frequency windows" at 15, 45, 60, 75, 90 and $105 \mathrm{~Hz}$ when the applied field value is kept at $42.5 \mathrm{Vpp} / \mathrm{m}$. Similar observations can be made about other values of frequency and field. [Blackman 85a] 
AC) magnetic fields present in the labortory setup. In a recent experiment, Bellossi has found that a replication of the efflux experiment with only static (frequency $=\mathrm{OHz}$ ) magnetic fields produces no effect.. [Bellossi 86].

Blackman has recently found that $16 \mathrm{~Hz}, 40 \mathrm{~V} / \mathrm{m}$ fields affect the efflux from brain tissue not only of calcium ions which are charged but also of the neutral sugar molecules, mannitol and sucrose. Further experiments are being carried out to see if the window pattern seen for calcium ions persists for these neutral molecules [Blackman 87].

Gundersen et al. [Gundersen 86] studied the effects of electric and magnetic fields on calcium efflux from cells of chick spinal cord. For exposure to $60 \mathrm{~Hz}$ fields of $1 \mathrm{G}, 30 \mathrm{kV} / \mathrm{m}$ for varying periods of upto 72 hours, no change was found between sham and experimental cases. [Gundersen 86] Because of the use of different cells - spinal cord rather than brain- and of a single pair of field values, these results should be viewed as a separate observation rather than an attempt to replicate the Bawin and Blackman work.

The calcium efflux experiments described above point up several peculiarities of the ELF-tissue interaction at least in this one effect:

. A. Frequency and Intensity Windows:

For a particular value of frequency, some field intensities produce the effect but others do not. Conversely, if an effect is observed at a particular value of field, it might be *'tuned out" by changing the frequency of the field.

The "windowed"* nature of these effects imply that when one looks at experimental results, one must bear in mind that even when there is no effect at some field values there may be effects at other, lower or higher values.

. B. Background static field conditions may matter:

The effect may be influenced by how the field is applied relative to earth's natural static magnetic field.

. C. "More is worse" paradigm does not hold:

For this cellular level phenomenon at least, it is not true that a larger value of the field shows a larger, or even any effect, compared to a smaller value of field.

The features described above have been observed in the cell in vitro. Blackwell and Reed of the National Radiological Protection Board in the United Kingdom attempted to see if the calcium efflux effect seen in vitro in the chick brain, could be detected through an effect in an intact animal [Blackwell 85]. They looked for signs of change in exploratory activity and of barbiturate-induced sleeping time in male mice exposed to 50 to $400 \mathrm{~V} / \mathrm{m}$ at 15,30 , and $50 \mathrm{~Hz}$, Both of these parameters are sensitive to changes in the central nervous system (CNS) associated with calcium changes. Their experiments failed to show any effect. The authors suggest that the results indicate that the fields used may not be of the right magnitude, or that the calcium efflux change that may have been induced in the brain by the fields were not large enough to produce changes in the CNS function. Blackwell [Blackwell 86] has also studied the activity of spontaneously firing neurons in anesthetized rat exposed to $100 \mathrm{~V} / \mathrm{m}$ field at 15 and $30 \mathrm{~Hz}$, both of which were resonant frequencies for the chick brain and for $50 \mathrm{~Hz}$ which is the frequency for power transmission in the United Kingdom. He found no change at $50 \mathrm{~Hz}$. However, the time, but not the rate, of firing was changed for 15 and $30 \mathrm{~Hz}$ signals. It is not clear what if any implication this has for the functioning of the animal.

Other than the work described above, no work has been done to see if similar effects exist in whole 
organisms and if they do, whether these too show windows in the effects. However, if biological effects of ELF fields on whole organisms show similar dependencies, then setting exposure standards would be a problem, because the "more is worse" maxim which applies for most agents such as environmental chemicals may not hold true in the case of ELF field exposure.

\subsection{Chromosomal Damage and Interference with DNA Synthesis and RNA Transcription}

DNA and RNA are the primary biomolecules in the cell. Nuclear DNA which is the primary constituent of the chromosomes carries the genetic code while the extranuclear RNA transcribe the DNA command codes into proteins for the physiological functioning of the cell. Well-studied cancer- initiating agents such as ionizing radiation and chemicals cause direct damage to DNA by mutations. As mentioned earlier, ELF fields do not have enough energy to break bonds or otherwise disrupt the structure of DNA.

Chromosomes from blood samples of mice chronically exposed to $60 \mathrm{~Hz}$ fields of $50 \mathrm{kV} / \mathrm{m}, 10 \mathrm{G}$, and from human lymphocytes and Chinese Hamster Ovary $(\mathrm{CHO})$ cells exposed in vitro were examined for sister chromatid exchange $(\mathrm{SCE})^{3}$ and other forms of chromosomal damage [Benz 87, Cohen 86, Livingston 86]. These experiments were all negative and the extensive nature of these studies leads to the conclusion that it is quite unlikely that ELF fields induce SCE's or other standard chromosomal aberrations.

However, changes in DNA synthesis rates and alterations in the transcription patterns of RNA, with the resultant production of structurally changed proteins have been observed in cells exposed to low intensity ELF fields. LibOff [Liboff 84] showed that the rate of DNA synthesis in human fibroblasts ${ }^{4}$ is increased on exposure to ELF magnetic fields with intensities comparable to the earth's magnetic field. Goodman and coworkers [Goodman 86] showed that $60 \mathrm{~Hz}$ fields both qualitatively and quantitatively change the pattern of translation (or, perhaps transcription) in cells from the salivary gland of a particular insect $^{5}$. The rate of production of the normal proteins made by the cell is increased. In addition, the field causes new proteins to be made. Protein synthesis is a very complicated process and the experiments yield no simple interpretation about the mechanisms or about potential effects on the organism.

\subsection{Interaction with Response to Hormones and Effects on Endocrine Tissue}

Only a few experiments have studied the effects of ELF fields on endocrine parameters. These involve responses of various tissues to the corresponding hormones in the presence of an ELF fields. Some interesting effects have been found. But, it is impossible at this point to draw any inference about the effects of fields on the endocrine system in a human or animal, other than to say that fields do exert an action on endocrine tissue and endocrine processes in vitro and these effects too show windows.

Luben et al. [Luben 82] reported a reduction in the cell response to parathyroid hormone (PTH) in

\footnotetext{
${ }^{3}$ SCE's are a well-characterized chromosomal defect known to result from agents such as ionizing radiation

${ }^{4}$ Fibroblasts are cells $i_{n}$ the "extracellular matrix" or the network of molecules connecting the space outside the cells. Fibroblasts are responsible for producing material such as fibrin and other collagens, which form a large fraction of the matrix.

${ }^{5}$ Protein synthesis is a complex process that begins in the cell nucleus. The first step is transcription, the Process by which "he information in the DNA in the genes is used to form the molecule mRNA (messenger RNA). After some modifications, this mRNA is translated into a sequence of aminoacids which rearrange to form proteins.
} 
mouse cranial bone cells, exposed to $72 \mathrm{~Hz}$ and $15 \mathrm{~Hz}$ pulsed magnetic fields. This response was measured as reflected in the cyclic AMP (cAMP) accumulation and collagen synthesis which normally result from administration of $\mathrm{PTH}^{6}$. Although not a $60 \mathrm{~Hz}$ field experiment, this study is significant in that it demonstrated rather clearly that, for this action at least, the membrane is the site of action.

The only studies on endocrine tissue in vitro have been done by Lymangrover and coworkers who investigated the effects of $60 \mathrm{~Hz}$ electric fields on the adrenal tissue ${ }^{7}$ (Lymangrover83, 87). They found that a $60-\mathrm{Hz}$ electric field caused an increase in the production of corticosterone in response to the hormone ACTH (adrenocorticotropical hormone). Corticosterone is a hormone of the steroid family involved in stress response and in anti-inflammatory reactions of the body. ACTH is a hormone one of whose functions is to stimulate the production of corticosterone by the adrenal tissue. In the present context, the production of corticosterone in response to ACTH is used as a biological process to examine a possible effect of electric field exposure on a specific tissue function. The tissue level effect noted below cannot be simply interpreted in terms of consequences in the whole animal.

The field did not produce any change in the amount of hormones produced by the tissue alone (basal activity of the tissue). But when the tissue was activated with ACTH, and the resulting production of corticosterone was measured, there was an increase in corticosterone production for certain values of the field, for certain durations of exposure. That is, the effect showed intensity windows combined with an exposure time dependence. Specifically, a $10 \mathrm{kV} / \mathrm{m}$ field intensity produced a fourfold increase in corticosterone production on 5.5 to 7 hours exposure and a $1,000 \mathrm{kV} / \mathrm{m}$ intensity produced a twofold increase during 2 hours of exposure. Fields of 5 and $100 \mathrm{kV} / \mathrm{m}$ produced no effect for any of these durations. The complex intensity and time dependence are the most notable features of these experiments. Although corticosterone level increase in response to ACTH is a stress response, tissue level experiments do not necessarily act as indicators of response in situ [Axelrod 84].

\subsection{Interaction with the Cell Response to Neurotransmitters}

As much of the activity in the brain takes place through electrical signals, it is reasonable to examine whether some of the chemical production in the brain are affected by exposure to electric fields. Rates of neurotransmitter secretion especially of norepinephrine and dopamine by the hypothalamic section of the brain in chicks and rats were found to change upon exposure to electric fields [Vasquez 86]. This effect is detailed in the subsection 4.2 on experiments in the whole animal.

\subsection{Interaction with Immune Response of Cells}

The immune response of the cells, that is the ability of the cells to act against foreign agents such as viruses and toxic chemicals, is important in combating infection and maintaining a healthy function. The several types of blood cells responsible for immune response have been examined in electric fields to see if any change in their function can be observed. Decreased immune response could lead to decreased resistance to disease or other harmful agents and is also suspected of accelerating the growth of some cancers.

\footnotetext{
${ }^{6}$ Cyclic AMP or_cyclic Adenosine Monophosphate, is an intracellular signaling molecule, or, "second messenger"' like calcium. PTH is a hormone that activates bone absorption of calcium using cAMP as the second messenger.
}

7Tissue from adrenal glands, situated over the kidney 
Winters examined human and canine leukocytes ${ }^{8}$ under different combinations of ELF electric and magnetic field exposure to see if immune response is modified by field exposure. [Winters 86]. Leukocytes taken from these dogs and people were examined under two conditions. First, immune properties of normal, unstimulated white blood cells (that is, healthy white blood cells that was not exposed to any known challenge to the immune system) were measured during exposure to fields. Second, the dogs and human volunteers were injected with antigens ${ }^{9}$ and the white blood cells thus stimulated to an immune response. The cells were then exposed to combinations of electric and magnetic fields. The exposed cells were examined for immune responses. Tests showed no significant effects of ELF field exposure on immunologic functions of normal or specifically immunized cells.

It is worth noting in this context that some authors have found responses when the cell was first stimulated by antigens and then exposed to fields, and when the cells were subjected to pulsed fields ${ }^{10}$ Most of the results discussed in this paper are for fields which are not pulsed [Chiabrera 84, Hellman 85]. These observations point to two possibilities: that field effects on immune system response are complicated ; and that there is a marked difference between cell behavior in sinusoidal regularly alternating) and pulsed fields.

Lyle (1986) reported an alteration in the cytotoxicity (that is, the ability to kill cells) of T-lymphocytes of the type CTLL-1 which are purified mouse lymphocytes that specifically attack cancer cells. The cells were exposed to $60 \mathrm{~Hz}$ electric fields for 48 hours and their cytotoxicity measured. In general, the fields inhibited the ability of these cells to kill cancer cells. A $0.10 \mathrm{mV} / \mathrm{cm}$ field inhibited the ability by $7 \%$, a field of $1 \mathrm{mV} / \mathrm{cm}$ by $18 \%$ and a field of $10 \mathrm{mV} / \mathrm{cm}$ by $30 \%$. In this experiment, therefore, one sees a "doseresponse" type of relationship between the effect the magnitude of the effect and the intensity value of the applied field. That is, in this range, the effect (inhibition of cytotoxicity) increases in proportion to the intensity of the applied field. If the reduced immune capacity exhibited in this experiment by one particular type of lymphocytes when exposed to low intensity electric fields, against a particular type of cancer cell) turns out to be present also when the cell is in the whole organism, it may be one mechanism by which the field inhibits the body's resistance to cancer or cancer growth.

\subsection{Interaction with Cells Relevant to Cancer}

One hypothesis for potential carcinogenesis by ELF fields is that the fields promote cancer formation or cancer growth rather than initiating cancer. This is discussed in the Endnote 2 on cancer. The fact that ELF fields have not been known to cause alterations in DNA structure (see Section 3.2), is consistent with the observation that ELF fields do not initiate cancer.

Several authors have looked for a response of leukemia cells to ELF field exposure [Liboff 84, Winters 86, Cain 86]. LibOff and Kaplow found that mouse leukemia cells exhibited increased DNA synthesis [Liboff 84]. Winters and Phillips found increased DNA synthesis in human colon cancer cells after 24 hours of exposure to $60 \mathrm{~Hz}$ magnetic field. Field-exposed cancer cells also showed an increased

\footnotetext{
${ }^{8}$ Leukocytes is the general term for white blood cells, types of which destroy invading bacteria, modulate allergic reactions, evoke several immune responses and kill some infected and tumor cells.

${ }^{9}$ Antigens ar ${ }_{e}$ substances that elicit an immune response from the body. Examples are infection-causing Viruses, pollen, etc.

${ }^{10}$ Pulsed fields are fields which are turned on quickly for only a brief period. Most of the results discussed in this paper are 'or fields which ar not pulsed.
} 
capacity to proliferate compared to unexposed cancer cells. At the request of the New York State Power Lines Study Project, which sponsored the Winters study, Cohen attempted to replicate the Winters results. [Cohen 871 . He found no significant effects of the fields to affect the proliferative ability of the same two lines of cells. For this and other reasons, the validity of the Winters results has been questioned.

The group at the J.B. Pettis Veterans Hospital at Loma Linda have examined the tumor promotion hypothesis by looking at effects of ELF fields on specific biochemical processes in the cell rather than by looking at the cell growth itself. Their results are described below. They have so far examined :

1. Decreased immune response depicted by the CTLL-1 experiment described in the previous section [Lyle 86],

2. Accelerated growth potential as measured by the increased activity of ornithine decarboxylase (ODC), described below [Cain 86, Byus 86],

3. Loss of the ability of cells to communicate because of the loss of gap junctions, described below [Fletcher 87].

Ornithine decarboxylase (ODC) is present in all cells and is an essential enzyme for cell growth because it helps synthesize biochemical that are necessary for DNA and protein syntheses. Any agents promoting cell growth also increase ODC activity. Examples of biochemical that cause increased ODC activity when administered to cells are : hormones and growth factors in the case of normal cells, and tumor promoters such as phorbol esters that promote uncontrolled growth of tumor cells. Hence factors that increase ODC activity can, but do not necessarily, lead to tumors. Highly increased ODC activity has been used as an indicator of malignancy.

Cain's experiments used normal fibroblasts which are a classical system for tumor promotion. There was a two-fold increase in ODC activity in cultured fibroblast cells exposed to $60 \mathrm{~Hz}$ electric fields at $10 \mathrm{mV} / \mathrm{cm}$.

Fletcher's experiments are based on the observation that gap junctions which help cells communicate with each other lose their function in many tumor models in which there is evidence for promotion. An example is the skin tumor in mice, a classical model of tumor promotion. He also notes that tumor promoters have no effect on cells that do not express gap junctions. Fletcher reported that while the field by itself has no effect on gap junctions in fibroblast cell lines, the field enhances the effect of the tumor promoter chemical TPA ${ }^{11}$. This experiment was, however, with modulated microwaves rather than with $60 \mathrm{~Hz}$ fields.

Byus exposed human lymphoma cells to $60 \mathrm{~Hz}$ fields and reported both intensity and time windows for increase in ODC activity. The same value of increase was noted for $10 \mathrm{mV} / \mathrm{cm}$ and $0.1 \mathrm{mV} / \mathrm{cm}$ field intensities, with no effect by field values in between. On continuous exposure to field, the effect was maximum at 1 hour of exposure time, and fell to control values at 2 hours of exposure. Beyond two hours exposure, ODC activity continued to decrease, so that the phenomenon changed from one of enhancement to one of inhibition of ODC activity after two hours. The activity went back to normal values when the field was removed [Byus 86].

\footnotetext{
${ }^{11}$ TPA or 12-o-tetradecanoyl phorbol-13-acetate, is a potent promoter of cancer often used as an experiment standard in cancer promotion experiments
} 
All the observations above, except the last, are consistent with a hypothesis that fields can promote tumors but again carry with them the warning that any potential relationship between the field intensity and the degree of promotion and may be highly complex. The last experiment shows that continuing to remain in the field once an organism has entered it may be less perturbing than a pattern that involves frequent periods of exposure and non-exposure .

\subsection{General Observations on Cell Level Experiments}

Despite many negative results, a significant number of cell level experiments have shown positive effects from exposure to ELF fields. These are summarized in Table 3-1. These results do not yet indicate any clear adverse impacts at the level of the whole animal. They do clearly demonstrate that:

. the cell membrane is one site of action of the field; and, therefore, processes governed by the cell membrane may be candidates for disruption by field exposure, the immune response and cell-cell communication being two such processes;

. the fields do not appear to act directly on the DNA structure; but may alter cellular processes by interfering with the transcription by RNA, a process in the chain of command from DNA to protein production;

. the effects may show a complex dependence on the intensity and frequency of the field and the time pattern of exposure to the field; further, the effects of fields may depend on the direction of the applied field in relation to the earth's magneitc field or, upon whether the field is a simple alternating field or a pulsed field. (i.e., a field turned on rapidly for brief periods) 
Table 3-1: Cellular Level Experiments: Effects and possible significance A Summary of results described in this section

Calcium efflux from cell membrane (6 experiments)

Chromosomal Damage (3 experiments)

DNA Synthesis Rate (1 experiment)

RNA Translation

(1 experiment)

Cell Response Modifications: Response to:

A. hormones

(1 experiment)

B. Neurotransmitters

(1 experiment)

C. immune system

(5 experiments)
Efflux is dramatically changed. The change occurs only at some frequency and intensity values, but not at others.

No chromosomal damage detectable.

Rate change at low magnetic field.

New proteins made by the cell. Rate of transcription altered.

Modifications in adrenal and bone tissue and connective cell response. to hormones

Phase shifts in the periodicity of secretion rhythms

Not clear that there are significant effects except in special cases.
Significance not clear But points up the possibility that effects of fields may not be such that "higher field intensity is worse than lower".

Does not cause the damage that usually initiates cancer.

Extremely low AC magnetic fields as small as the earth's natural DC field may affect cell process rates.

Fields may alter rates of primary cell processes.

Significance not clear. Adrenal response shows intensity windows. Bone tissue experiment points to membrane as site of action.

If true in humans, might have implications for psychological disorders, such as chronic depression.

Implications not clear. 


\section{Whole Animal Experiments}

Animal systems have been examined under a range of electric and magnetic field intensities and for varied exposure conditions and durations. Historically, the animal experiments began by looking for general effects rather than by formulating and testing hypotheses because there was no indication about what system or function, if any, is most likely to be affected by fields.

The very early experiments in this area were riddled with problems of poor experimental design leading to artifacts in results. Because of this, studies prior to 1970 are not discussed in any detail in this report. In the past fifteen years, there have been a set of high quality experiments. But, as in the case of the cellular level experiments, a hypothesis testing stage has not been reached in the science of health effects on animals or humans. Epidemiological studies have focused on a search for cancer because of a historical observation and the public saliency of cancer rather than because cancer is thought to be the most likely effect.

Among the most impressive and concerted set of experiments with animals have been those conducted at the Battelle Pacific Northwest Laboratories in Richland, Washington [Phillips 79] under a rather large project funded primarily by the Department of Energy, and a few smaller projects by Electric Power Research Institute (See Section 8), The first phase of these experiments consisted of extensive screening studies in which animals were examined for all kinds of effects of electric fields at the perceptual, behavioral and physiological levels. This screening was done under fairly high field strengths, much higher than any field that is likely to be encountered even under the right-of-way as it was thought that this would enable a potential effect to be picked up. This argument assumed that exposure to higher fields produce more pronounced effects than that to lower values. The Battelle studies used small animals such as mice, rat and miniature swine to study effects of fields on behavior, physical and motor development and growth, immunology and hematology, and endocrinology. Several of the studies spanned generations. The breadth of these studies, their careful experimental design and interpretation make them the central source of knowledge in this area. This has in large part been due to the excellent team work by experts in the various fields relevant to this area of study such as neurochemistry, stress physiology, psychology, developmental toxicology, electrical engineering and physics and careful integration of the results. Animal studies with sufficient numbers of animals to get statistically significant results, are very expensive and time-consuming. For example, some of the Battelle studies spanned two or three generations of mice and pigs ( $4-5$ years), and involved as many as two hundred rodents in each study.

Other laboratory studies with whole animals have involved isolated experiments rather than an extensive program. Most of these have been funded by the New York State Power Lines Project (NYSPLP), and by the Department of Energy program described in Section 8. Examples are neuroendocrine studies by Michelson et al., circadian rhythm studies on rats by Ehret et al., and on monkeys by Sulzman et al.; on field avoidance behavior of rats by Sagan et al., by Lovely and by Hjeresen et al.; on central nervous system effects by Thomas et al. and Ossenkopf et al.; and development in rats by Walker et al. and Salzinger et al. References are given below as each study is described. Several studies among these were funded by the five-year long New York State Power Lines Project. We have not described the avoidance behavior studies because it is not clear that they have relevance to health effects, especially in humans.

Most well-designed animal studies expose the experimental animal to the fields according to a set 
protocol of field exposure in a controlled environment. Exposure chambers for the experiments have to be specially developed and calibrated. great care must be taken to avoid factors such as vibration, hum, ozone or shock which could lead to erroneous results or "artifacts". Exposed animals are compared with animals living in an identical environment but subjected only to a "sham" exposure, that is, all conditions for exposure are simulated but there is no actual exposure. The Battelle studies and some of the others were done "blind" so that the persons handling and recording results from the animals did not know which animals were really exposed and which were sham-exposed.

Field studies have been done on cows and some small animals in Sweden and Italy and by the Bonneville Power Administration in Oregon.

Some studies of human physiological parameters have been done as part of the New York State Power Lines Project. These are outlined in a separate section below. Finally, there are several epidemiological studies that have investigated an association between ELF field exposure and cancer. These are discussed in Section 6.

The animal and human studies are now reviewed under the following categories of effects:

1. General effects such as detection, avoidance and behavior responses and development and learning of animals, and moods of humans.

2. Effects on externally measured physical parameters such as growth and birthweight, respiration, heartbeat rate, and temperature rhythms.

3. Effects on specific biochemical such as hormones, and blood components

4. Effects on reproduction, growth and development

5. Effects on circadian rhythms of animals and humans.

6. Effects on human perception, performance on specific tests and physiology

7. Epidemiology of cancer, particularly leukemia and brain cancer.

\subsection{Detection, Behavior, Learning and Avoidance Responses in Animals}

Early Russian reports of necrologic symptoms such as headache and fatigue [Korobkova 72] together with the supposition that nervous tissue might be most responsive to field exposure because some of its functions are enabled through electrical signals, prompted several of the animal experiments. These experiments studied central nervous system (CNS) function directly by the observation of behavior or indirectly by measurements on the secretion of certain hormones known to be associated with effects on the central nervous system.

Perhaps the largest set of behavioral studies have involved studies of animal preferences to remain in or avoid strong electric fields [Hjeresen 80, Stern 83, Stern 85]. While they are interesting, and show, for example that rats can detect fields of just over $1 \mathrm{kV} / \mathrm{m}$, we do not believe these experiments provide much insight on health effects. They do, however, suggest that effects observed in experiments involving strong electric fields may reflect the effects of sensory stimulation, rather than the direct effects of fields on the cell.

Rogers and Smith have conducted experiments on the behavior of baboons in strong electric fields of $30 \mathrm{kV} / \mathrm{m}$ and $60 \mathrm{kV} / \mathrm{m}$. While the baboons exhibited alterations in postures and positions, there appeared to be no consistent trend and the changes in positions were noted to be temporary. [Rogers 87 ] 
No perception mechanism for magnetic fields at ambient strengths is known .

Thomas, Schrot and LibOff [Thomas $86 \mathrm{a}$, Thomas $86 \mathrm{~b}$ ] studied the effects of magnetic fields alone or in combination with drugs on specific learning schedules of rats. The objective was to look for effects on behavior and learning and to see if fields affect the action of certain drugs on these animals. Magnetic fields of $0.5,1,3$ and 5 gauss were used, alone or in combination with $1 \mathrm{kV} / \mathrm{m}$ electric field. The results indicate no systematic effect on the behavioral pharmacology of the two drugs used. Both of the drugs have very definite reproducible effects on the learning schedules used. In $60 \mathrm{~Hz}$ magnetic fields alone or in combination with electric fields, the learning schedule effects of the drugs continued to be the same as in the absence of the field.

However, when the $60 \mathrm{~Hz}$ magnetic field was combined with a static magnetic field, a systematic reproducible modification was produced in one of the learning effects. The change is transitory, lasting more than one hour, but disappearing within 24 hours even when the field exposure is continued. The authors conclude that under very precise conditions, magnetic fields may have an effect on learning.

Ossenkopp and Kavaliers showed that exposure to $60 \mathrm{~Hz}$ magnetic fields in the range 1 to $1.5 \mathrm{G}$ produces a significant reduction in epileptic seizures induced in rats by pentylenetetrazol, an epileptogenic drug [Ossenkopp 86]. There is no effect at 0.5 and $1.85 \mathrm{G}$. Exposure to $1 \mathrm{G}$ also had a weak inhibitory effect on development of seizures by electrical kindling, that is, bringing on epileptic seizure by applying an electric current to the brain. Mice exposed to magnetic field in the range 0.5 to $1.5 \mathrm{G}$ also exhibited significantly less analgesia than control or sham-exposed groups when morphine was administered to them. That is, exposure to magnetic field appeared to make the rats less sensitive to the numbing effects of morphine. The degree of inhibition increased for higher fields. However, the fields did not influence the tolerance development to morphine in the mice.

These observations can not be simply extrapolated to any general conclusion except to note that there are central nervous system effects which may be windowed even in the whole animal, and be very specific with respect to field values as well as to specific functions.

\subsection{Effects on Hormone Levels and the Central Nervous System}

Levels of specific hormones are maintained in balance for the proper functioning of the central nervous system (CNS) (See Endnote 2 for discussion of the specific hormone systems). Most prominent among the aminoacid hormones synthesized in the brain and the nervous system are the catecholamines dopamine, norepinephine and epinephrine. As they are released from nerve endings during physiologic stimulation, they are called neurotransmitters. They have several functions and affect muscle, heart, liver, spleen, lung and brain physiology. They control blood pressure, heartbeats, some forms of headache, the basic metabolic rate, some psychological changes and several other rates. Deficient or excessive secretion of catecholamines or alterations of their action cause major physiological and psychological problems. Increased metabolism of catecholamines is also associated with neuroblastoma, a tumor originating in the neural crest, often during fetal development.

Serotonin stimulates or inhibits many of the muscles and nerves, depending on the amount and the phase of the organ in its function. it can stimulate or depress heartbeat, contract blood vessels and change blood pressure. Serotonin prevents clotting, and provides reflexes such as coughing or hyperventilation. In humans, serotonin also serves as a chemical transmitter in the brain. Serotonin and 
its product melatonin influences sleep, perception of pain, psychological depression and social behavior. Melatonin is also secreted by the pineal gland and is described in the endnote on circadian rhythms.

The levels of these hormones are not only responsive to external and internal signals, but undergo a regular pattern of variation in different periodic rhythms. One such rhythm is the circadian rhythm which has a period of about 24 hours (See endnote 4 on circadian rhythms). Large variations of these levels and rhythms are therefore indicative of perturbations of CNS function.

In a study at Battelle, Free et al. (1981) noted a phase shift of the normal variations of hormones in adult male rats exposed to $64 \mathrm{kV} / \mathrm{m}$ for 30 days and 120 days, but no change in body or organ weights. They concluded that continuous exposure to electric fields may alter some endocrine system secretions but do not appear to impair normal physiology [Free 81]. No significant changes were noted in the levels of various neuroendocrine secretions by rats for 1 or 3 hours at $100 \mathrm{kV} / \mathrm{m}$ by Quinlan et al. [Quinlan 85].

A pineal function change was seen in the Battelle studies by Wilson et al. (1981). Continued exposure to $60 \mathrm{~Hz}$ electric fields of 1.7 to $65 \mathrm{kV} / \mathrm{m}$ produced a depression of the night-time levels of melatonin in rats, and introduced a delay in the activity in the rhythms of other biochemical activity. This pineal melatonin depression effect did not appear in the animals immediately on exposure. For a $39 \mathrm{kV} / \mathrm{m}$ field, the onset of the effect appears after two and before three weeks of exposure. In less than three days after the cessation of exposure, the levels returned to normal. This experiment is discussed again in the section on circadian rhythm because of its importance in that context.

Investigating this delay in the secretion of the neurochemicals in detail, Vasquez and coworkers working jointly with the Battelle group, have found that the daily periodicity (or, circadian rhythm) of some pineal hormones such as melatonin undergoes a change in rats exposed to strong electrical fields. A period shift of four hours has been observed for the hormones norepinephrine, serotonin and dopamine in the exposed rats. [Vasquez 86]. This shift in the time of occurrence of peak and low levels, called "phase shift", may or may not be present in humans. A phase shift of these hormones with respect to the natural light-dark cycle, if present in humans, might have implications for certain psychological disorders of biochemical origin such as sleep and mood disorders, and chronic depression. As described in Section 5 (Figure 5-1 ), the fields in these experiments generate in the animal a current density comparable to the exposure situation in a home near a $500 \mathrm{kV}$ line.

CNS endocrine secretion effects were also seen by Wolpaw et al. in an experiment on pig-tailed macaque monkeys [Wolpaw 87].. The animals were exposed to electric and magnetic fields from $(3 \mathrm{kV} / \mathrm{m}$ and $0.1 \mathrm{G})$ to $(30 \mathrm{kV} / \mathrm{m}$ and $0.9 \mathrm{G})$ for three periods of 21 days each, with 21 days of sham exposure in between. Weight, blood chemistry, blood counts and performance on a simple motor task as well as postmortem examination of brain and organs showed that fields did not apparently affect any of these factors significantly for the period of exposure. Spermatogenesis was also seen to be normal. The monkeys viewed by videotape while in the fields showed no indication of disturbance.

Cerebrospinal fluid examination at the end of each period showed significant decline of homovanillic acid (HVA) and 5-hydroxyindoleacetic acid (5-HIAA) whose levels in turn reflect those of dopamine and serotonin (See endnote 2 on hormones). The experiments did not show any circadian shifts. The 5-HIAA concentration failed to return to original levels after exposure, showing a permanent effect in modulating an important neurotransmitter system which also plays a role in several psychochemical disorders as mentioned above. 


\subsection{Effects on Blood and Immune System Chemistry}

Blood and serum chemistry can reveal abnormal levels of metabolizes, immune system components or hormones which may be indicative of improper functioning of some organ system. Blood chemistry of rats exposed continuously to unperturbed fields has been examined in several studies.

A Battelle study looked at specific components of the immune system in mice in $68 \mathrm{kV} / \mathrm{m}$ fields for 30 to 150 days. It found no systematic significant variation in counts of blood cells or antibody responses [Morris 82]. In another study at Battelle, adult rats were exposed for 15,30,60 or 120 days to a field of $68 \mathrm{kV} / \mathrm{m}$. Blood counts of cells necessary for proper immune responses as well as serum constituents indicative of proper level of metabolism were studied in detail in replicated experiments on groups of rats. No variation was observed between exposed and sham-exposed rats in the various parameters examined [Ragan 83]. Studies on immune response of rats at a field level of $100 \mathrm{kV} / \mathrm{m}$ also showed no effect [Morris 87]. In independent experiments, Michelson et al. at the University of Rochester also found no change in these parameters [Quinlan 85]. The experiments on monkeys by Wolpaw et al. mentioned in the preceding section also showed normal hematology and serum chemistry.

These experiments imply that there is no general or overall immune system performance changes or endocrine system changes induced by exposure to electric fields of a rather high intensity over a duration of several months.

\subsection{Effects on Reproduction, Growth and Development}

Reproduction, growth and development studies measure a wide variety of factors including: reproductive behavior such as mating and fertility; the viability of the fetus; alterations in physical parameters such as birth weight and head size; gross malformations; and central nervous system development. In addition, some experiments have studied adult growth, particularly of bones and the ability of bone to repair after it has been fractured. These are now discussed under the different categories of reproduction and pre- and perinatal external development; central nervous system development and adult growth and repair capability as examined under field exposure,

\section{Reproduction, prenatal and perinatal development}

Most of the studies of possible developmental effects of ELF field exposure have concluded that there are no overt defects and malformations as a result of exposure. However, some studies have seen subtle effects and the possibility of the existence of effects remains an open question. Tables 4-1 and 4-2 summarizes the experimental results.

Several investigators have used chicken eggs to study the effect of ELF fields on embryonic development. While early experiments in Spain, using pulsed $^{12}$ magnetic fields reported an increase in developmental abnormalities [Ubeda 83, Delgado 82], attempts to replicate the effects yielded mixed results. Tell and coworkers at the U.S. Environmental Protection Agency could not see any statistically significant effect [Martucci 84]. However, Mild reported from Sweden that he did observe an increased abnormality in chick embryos exposed to weak, pulsed magnetic fields [MWN 84]. Maffeo et al. also found no developmental effects on chick embryos exposed to pulsed ELF fields [Maffeo 84].

\footnotetext{
${ }^{12}$ Pulsed fields are fields which are turned on quickly for only a brief period.
} 
Table 4-1: Summary of reproduction, prenatal and perinatal development in rats, and chicks exposed to $60 \mathrm{~Hz}$ fields

\section{Animal and Exposure Conditions}

Chick eggs

incubated in $0-100 \mathrm{kV} / \mathrm{m}$

electric fields

Rats in $100 \mathrm{kV} / \mathrm{m}$ fields

Males and females exposed

Females exposed during pregnancy

Females exposed during pregnancy; exposure of newborn continued for 8 days

Fetal exposure beginning at three-fourths term to 25 day old newborn

\section{Observed Effects and Conclusions}

No effects on mortality, deformity or birth weight No physical developmental defects in chicks due to exposure in this range

No effect on mating performance or fertility

No effect on full-term development of appearance and size of fetus

No effect on litter size, stillbirths, birth weights, or early external neuromuscular or neurological development

Fewer stillbirths and neonatal deaths in exposed compared to sham-exposed animals. No observable changes in early neonatal development 
Todate, exposure to $60 \mathrm{~Hz}$ fields of chick embryos has not shown any significant abnormalities. Graves et al. [Graves 85] exposed over 20,000 eggs to a range of $60 \mathrm{~Hz}$ electric fields ranging from $\mathrm{O}$ to $100 \mathrm{kV} / \mathrm{m}$ through the 21 -day incubation period. They found no effects on mortality, deformity, or on weights of embryos or one-day old chicks.

Pulsed magnetic fields, however, continue to cause concern and controversy in the scientific community with respect to their potential detrimental effect on the development of chick embryos. Following the early work cited above, the U.S. Office of Naval Research and the U.S. Environmental Protection Agency sponsored six independent laboratories in the U. S., Canada and Europe in a planned series of experiments involving exposure of chick eggs to pulsed magnetic fields with the same set of characteristics. All the laboratories also examined the same endpoints. Exposed eggs were examined for fertility. Embryos were examined for abnormalities in development, and for growth. This project, nicknamed the "Henhouse Project" showed an overall increase in the proportion of abnormal embryos in the exposed group for all the laboratories taken together, although the exact proportion of abnormality differed from one laboratory to another. It is to be noted again that this result is for pulsed fields. Scientists cannot say what the implications are for $60 \mathrm{~Hz}$ fields and humans. The Henhouse Project group are planning further research on developmental abnormalities.

Three experiments of reproduction and development were done on large numbers of rats (over 100 rats in each study) as part of the early screening studies at Battelle. The animals were exposed to uniform $100 \mathrm{kV} / \mathrm{m} 60 \mathrm{~Hz}$ electric fields [Phillips 79].

The first experiment, replicated three times, examined reproductive behavior, fecundity and fetal development. The male and female animals were exposed to the field for six days. This did not affect their reproductive performance. A 30-day exposure of males and females prior to mating also did not affect mating performance or fertility. The females continued to be exposed during pregnancy. The appearance and size of fetuses were examined after 20 days of gestation (Full term for rats is about 22 days). In the second experiment, postnatal development subsequent to prenatal exposure was examined in four replicates. Exposure was begun on the first day of pregnancy and continued until the offspring were $\mathbf{8}$ days old, a total of 30 days. Parameters of physiological, behavioral and neuromuscular development were noted. Exposure did not affect litter size, incidence of stillbirths, or birthweights. No differences between exposed and sham-exposed animals were noted in morphological, neuromuscular or neurological development.

The third experiment of five replicates measured development as a consequence of exposure for 30 days, from day 17 of gestation to when the offspring was 25 days old. The same set of developmental measures as in the previous experiment were used. Significantly fewer stillbirths and neonatal deaths were noted in the exposed group in this case. No changes were noted in neonatal development.

The authors concluded from these experiments that exposure to high fields before and immediately after birth did not affect the growth or development of rats.

Two experiments in which rats were exposed prenatally to $80 \mathrm{kV} / \mathrm{m}$ fields during the last trimester of pregnancy indicate that such exposure may slightly retard postnatal development as measured by ear flap separation and eye opening and alter sexual differentiation. These experiments were, however, done using only about 15 experimental and 15 control animals each and the results are therefore are not conclusive. [Burack 84] 
A study of Hanford miniature swine was also a screening study to look at a larger and longer-lived species than rodents over multiple generations. The time course of the systematic study over three generations is shown in Figure 4-1 reproduced from the paper by Sikov [Sikov 87].

In the figure, $F_{0}$ denotes the original generation of the female swine. These lived in a $30 \mathrm{kV} / \mathrm{m}, 60$ $\mathrm{Hz}$ electric field for 20 hours each day for 4 months before they were mated with unexposed male swine, and continued living in the field while they were pregnant with the first, and in some cases with the second, set of offspring. The first generation $F$, females were bred to unexposed boars at 18 months of age and gave birth to the second generation $F_{2}$. In the figure, Teratology ${ }^{13} \mathrm{I}, \mathrm{II}$, III denotes the points in time at which detailed morphologic evaluations of the newborn were performed. This included external measurements, weight and observations of internal and external malformations. Prenatal embryonic loss was also noted.

Teratology I and III, of the first set of offspring of the original and of the second set of offspring of the first generation yielded no incidence of abnormal growth or malformations as compared with sham exposed swine. Teratology II of the second bred offspring of the original generation showed a significantly larger proportion of malformations than the corresponding sham-exposed group.

There was a tendency toward less prenatal mortality in the exposed group. That is, there were more live fetuses per litter in the field-exposed group than in the sham-exposed. There were no other significant differences. The authors conclude that while the experiment suggests that there is an association between field exposure and prenatal growth and development, no definite conclusions can be drawn.

Two replications of the experiment similar to the swine experiment were performed with rats as a followup to the swine study at Battelle [Rommereim 87]. The rats were exposed to a higher field $100 \mathrm{kV} / \mathrm{m}$ for 19 hours per day. An experimental protocol similar to the swine study over the three generations was used.

In the first of these experiments, an increase in birth defects in the second farrow of the original $\left(F_{0}\right)$ generation and a decreased fertility of the $F$, generation were noted. But these effects were absent in the second replicate study. Because of this, it is not clear whether the observed effects in the first experiment were due to a random variation rather than due to a significant effect of field exposure.

The scattered positive results in these experiments do not allow the possibility of an association between electric field exposure and gross musculoskeletal abnormalities to be ruled out. However, taken together, the studies do not provide strong support for the existence of an effect.

\section{$\underline{\text { Prenatal exposure in humans }}$}

Wertheimer and Leeper (Wertheimer 87) used birth announcements to examine the association between the use of electric blanket or electrically heated waterbed and irregularities in pregnancy outcomes. The authors noted a seasonal pattern indicating a higher abortion rate, longer gestation periods and lower birthweights in babies born to users of heated waterbeds and electric blankets.

\footnotetext{
${ }^{13}$ Teratology is the study of birth defects
} 


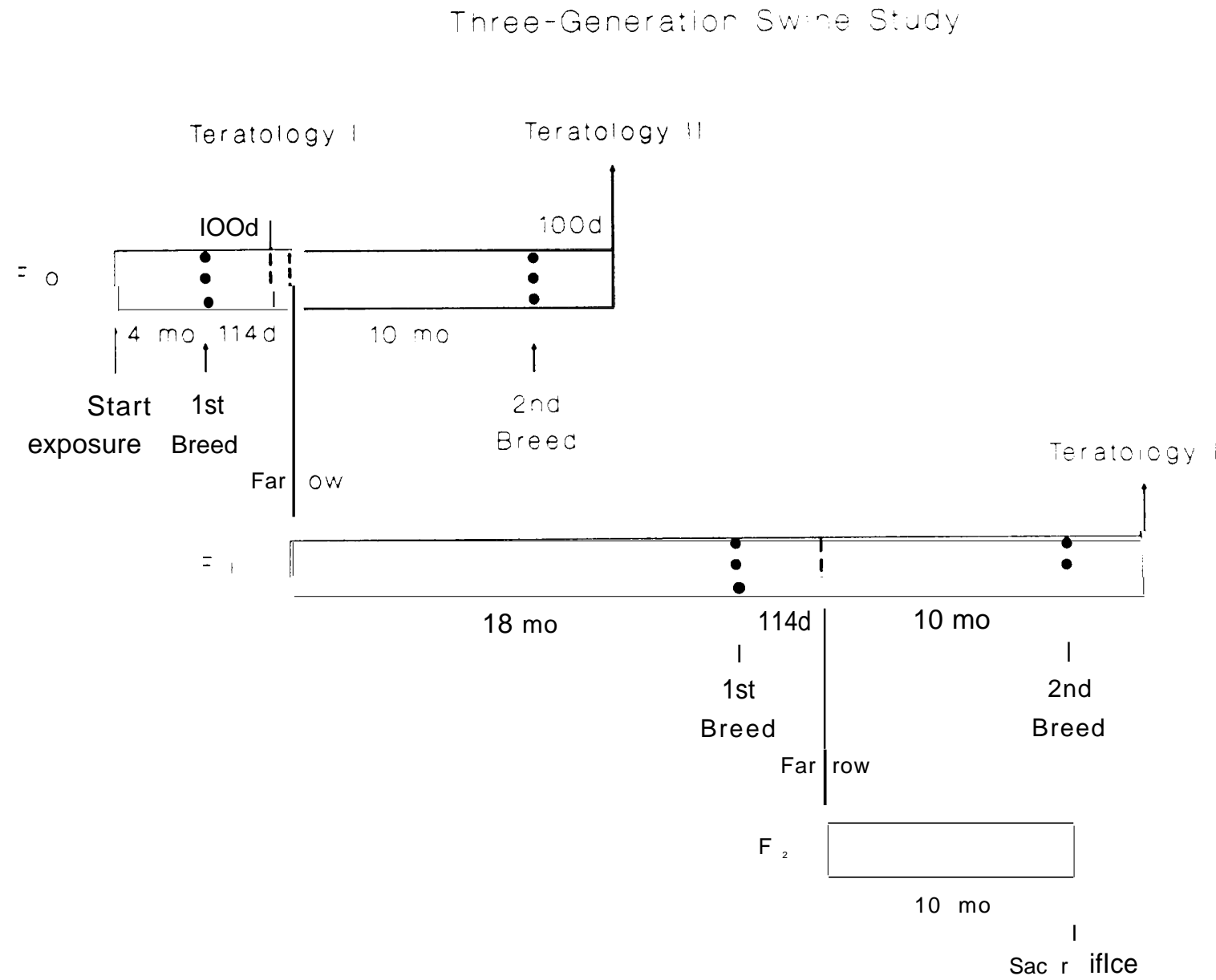

Figure 4-1: Scheme for three-generation reproduction study of Hanford miniature swine exposed to $60 \mathrm{~Hz}$ electric field of $30 \mathrm{kV} / \mathrm{m}, 20$ hours a day, 7 days a week: The horizontal length of the bars are proportional to the time elapsing in each generation. $F_{0}, F_{1}$, and $F_{2}$ denote the original, first generation and second generation of miniature swine respectively. Generations $F_{0}$ and $F$, were bred twice. After the start of the exposure, the female swine continued to live in the electric field. $F_{0}$, the original generation consisted of 49 sows derived from 13 mothers and a single father. 31 of these 48 animals formed the exposed group, and 18 the sham-exposed group. The $F_{0}$. sows were bred first after 4 months of exposure to unexposed boars. 18 exposed and 10 sham-exposed sows of the $F_{0}$ generation produced the $F_{1}$ generation. Teratology I (examination for birth defects) was done 14 days prior to birth on some of the litters of the $F_{0}$ swine. The $F_{0}$ were bred again after 10 months to produce the second farrovv of the original generation. The $\mathrm{F}$, females were bred at 18 months age to produce $F_{2}$ generation and again 10 months later to produce the second farrow of the first generation.

Reference: [Sikov 87] 
Table 4-2: Studies of Three Generations of Swine and Rats

\section{Animal and Exposure Conditions}

Hanford miniature swine Three generations in $30 \mathrm{kV} / \mathrm{m}$ electric field Females exposed and mated with unexposed boars

\section{Observed Effects and Conclusions}

First set of offspring of original and second set of offspring of first generation normal in prenatal development. Less prenatal mortality in exposed group.

Second bred offspring of original generation showed increased incidence of malformations primarily of musculosketal system.

Rats: Replication

of swine study. Two repetitions of three generation study

Study 1 Increase in birth defects of second farrow of first generation parallel to swine study

Study 2

No effect observed. 
This study is at best a preliminary scoping study because of the large number of experimental biases and small sample size. Sources of human prenatal developmental abnormalities are characterized only for at most 20 to $30 \%$ of the observed cases [Wilson 74] and confounding effects abound. Only a very careful study done with a knowledge of the base seasonal variations of different kinds of abnormalities, maternal habits and exposures, and genetic susceptibility can reveal any potential effect of fields on the developing human. A database for the baseline for such a study exists in the Congenital Malformation Surveillance System at the Center for Disease Control in Atlanta [Edmonds 81 ]. This is an intensive surveillance system which is a useful resource for birth defect studies, especially in the case of weak potential agents such as ELF fields. On our initiative, selected questions related to ELF exposure have been added to the survey questionnaire for this system since late 1985 [Sever 84] and may yield reasonable conclusions by the early 1990's. The questions ask about the use of electric blankets and electrically heated waterbeds during and immediately preceding pregnancy in order to assess if there is any observable association between such use and birth defects.

Growth, development and bone repair in adult $\underline{\text { animals }}$

Growth and repair in bones have been associated with pulsed ELF fields used clinically [Bassett 74, Bassett 82] and several studies have examined the effects of $60 \mathrm{~Hz}$ fields on bone growth and repair.

In a large study at Battelle, Hilton and Phillips conducted a series of 31 separate experiments, 17 with rats and 14 with mice to determine effects of $65 \mathrm{kV} / \mathrm{m}$ fields for different periods of exposure $(30,60$, or 120 days) [Hilton 81]. Effects examined were: body and organ weights; food and water consumption; and, metabolic rates such as oxygen consumption and carbon dioxide production. While there were some variations in some of the experiments, the authors concluded that there were no major changes in any of these features and that therefore living in $65 \mathrm{kV} / \mathrm{m}$ fields for four months produced no effects on the growth and metabolism of rodents.

A smaller study by Walker et al. [Walker 82] examined effects on bone growth in prenatally exposed rats. Leg bones from 55 adult rats conceived, born and raised in a $80 \mathrm{kV} / \mathrm{m}, 60 \mathrm{~Hz}$ electric field were compared with those from sham-exposed animals. Body weight, wet and dry bone weights, bone length, and specific gravity of bone were studied. Chronic field exposure enhanced growth in the long bones causing increased length and mass, but not in specific gravity. No tumors or other effects were noted. The magnitude of the effect was small, about $5 \%$, but statistically significant. In another study, male rats were exposed to $10.2 \mathrm{G}$ at $60 \mathrm{~Hz}$ for 30 days. Although there appeared to be a trend toward impaired growth, there were no observable skeletal effects [Simmons 86].

The Battelle group also studied the effect of $100 \mathrm{kV} / \mathrm{m}$ fields on bone growth and fracture repair [McClanahan 83] in rats. Exposure of young and adult rats of both sexes for 20 hours a day did not alter growth rate of bones. Bone repair was tested by examining bending and breaking strength of fractured and intact leg bones at several periods after bone surgery. The exposed group showed a slightly lower repair capability as measured by lower strength during early periods after surgery( 16 and 20 days), but the repair was normal by day 26 . The authors state that the exposure retards the rate of repair but does not lead to permanently diminished strength. It is also not clear whether the diminished repair was due to the lowered rate of bone deposition or due to the fact that the rats in the field tended to move more than the sham-exposed rats because they felt the field. 
Overall, high intensity electric fields do not appear to have a strong effect on bone growth and repair in rodents.

Early brain and central nervous system $\underline{\text { development }}$

There are several isolated experiments that have looked at different effects which are indicators of early development of the brain and central nervous system. Because of the different aspects examined, they do not lead to any simple conclusion. The results are summarized in Table4-3. The one significant lesson from these experiments is that there maybe subtle effects in facets of development that are hard to measure. These may include learning retention as the animal is subjected to repeated learning experiments. All but one of the studies have done single measurements of some parameter of learning rather than continued repetitive learning.

Studies in Sweden by Hansson reported that the brain morphology of certain parts of the brain of various small animals such as rodents and rabbits born and reared under $100 \mathrm{kV} / \mathrm{m}$ fields showed abnormalities [Hansson 81]. These results were based on qualitative tissue pathology and were subject to significant criticism in the research community. Experiments since then have failed to replicate the result [Albert 84].

As part of the New York State Power Lines Project (NYSPLP), a study by Gona et al. examined the various aspects of the developing nervous system in rats [Gona 871. The prenatal and perinatal development of rat brain was studied under continuous exposure of pregnant and newborn rats. Three field combinations ( $1 \mathrm{kV} / \mathrm{m}$ and $10 \mathrm{G}) ;(100 \mathrm{kV} / \mathrm{m}$ and $1 \mathrm{G})$; and, $(100 \mathrm{kV} / \mathrm{m}$ and $10 \mathrm{G})$ were used. Indexes of cerebral and cerebella development of the brain were noted for different periods of development.

No significant permanent differences in the biochemical and morphological parameters of brain development were observed between exposed and sham-exposed animals. The features observed by Hansson were not evident in these animals.

Blackman et al. [Blackman 88] examined whether the in vitro calcium efflux effect in chick brain tissue described earlier in section 3 is affected by prenatal exposure of the chick. (i.e., the exposure of eggs) to $60 \mathrm{~Hz}$ and $50 \mathrm{~Hz}$ fields. They found that eggs exposed to a $60 \mathrm{~Hz}$ electric field produced chicks with brain tissue that showed the calcium efflux effect for 50 but not $60 \mathrm{~Hz}$. Eggs incubated at $50 \mathrm{~Hz}$ produced chickens with brain tissue that were not affected by 50 or $60 \mathrm{~Hz}$ fields. The result was not sensitive to particular intensity values, but to the frequency. This finding was confirmed in three separate experiments by Blackman. It shows that prenatal exposure to ELF fields can affect postnatal tissue physiology in very specific ways.

Subtle central nervous system effects can result from some prenatal insults that may be manifested only later in the offspring's life and hence may not become evident in studies done soon after birth. Examples of prenatal chemical insults that lead to defects much later on in life are the pregnant mother's use of diethylstilbesterol (DES) which led to carcinogenesis in women offspring; and of alcohol use that can lead to learning deficits in children and nutritional deficits that lead to persistent functional deficits [Snell 82].

The Battelle studies described earlier in this section have examined some learning and behavioral 
Table 4-3: Early development of brain and central nervous system in exposed animals (Details in text)

\section{Experimental Conditions}

Rodents and rabbits born and reared in $100 \mathrm{kV} / \mathrm{m}$; Brain tissue examined for change in appearance

Prenatal and perinatal development under combinations of $1 \mathrm{kV} / \mathrm{m}$ and $100 \mathrm{kV} / \mathrm{m}$ electric field, and $1 \mathrm{G}$ and $11 \mathrm{G}$ magnetic field

Calcium efflux effect in brain tissue of chicks hatched from exposed eggs

Rats exposed from 3/4 term of gestation to 11-20 days age

Early learning and repetition of learning in rats exposed in utero
Observations and Conclusions

Alteration noticed on examination by Hansson in Sweden. Attempts at replication by Albert in the U.S. found no evidence of the differences.

No significant effects on biochemical or visual morphology of brain development

Frequency window may be affected by prenatal exposure. No intensity window

No effect on early motor activity learning;

No effect in total electrical activity of the brain

Learning response decreases when learning tests are repeated. 
aspects of newborn rats as well as perinatally (day 11-20). In the simple learning experiments involving motor development, no significant effects were found.

in another set of experiments at Battelle, Jaffe [Jaffe 83] measured the development of the visual evoked response (VER) in newborn rats exposed prenatally and perinatally. The VER is a measure of the total electrical activity of the nerve cells that process the information supplied by a visual stimulus (regular light flash in this instance). Early changes are an index of CNS development. In tests on a total of 114 rats from 114 litters, no significant effect of exposure was found for upto 20 days of perinatal exposure.

Salzinger et al. note that it may be important to study behavioral effects over more training sessions than may be done ordinarily [Salzinger 87]. In their studies, they examined the behavior of rats exposed in utero and for the first eight days of life. Exposure levels of $30 \mathrm{kV} / \mathrm{m}, 1 \mathrm{G}$ and $10 \mathrm{kV} / \mathrm{m}, 0.33 \mathrm{G}$ fields were used and the experiment was done blind, with sham and real exposure settings.

A specific behavioral and learning test was used. Other parameters such as physical appearance, general activity level and weight were also monitored. None of the physical parameters showed any difference between the sham and exposed groups at either of the field levels. The exposed and shamexposed group started out with the same learning response level. However, repetition of the learning tests showed that the performance of the two groups increasingly diverged, with the exposed group showing a more and more lowered rate of response in subsequent sessions. This divergence was higher for the higher field levels than the lower. This difference in learning was found to be robust in that it was maintained when the behavior was extinguished and the animals reconditioned. The effect was reproducible.

Salzinger et al. note that most experiments involve an examination of the early learning behavior and do not continue conditioning studies. Their results show that it is important to study conditioning over a larger number of training sessions because the effects may become evident only late in the conditioning process. The robustness of their results implies that it may be important to attempt to replicate experiments of this type in other laboratories, in other animals and for other learning behaviors in order to understand the full implications of perinatal field exposure for learning.

\subsection{Effects on the Circadian Systems of Animals and Humans}

The circadian timing system serves to synchronize various physiological and biochemical processes that have a daily cycle. While many aspects of the biology of the circadian and other timing systems are not well understood as yet, the last two decades have brought considerable understanding of some of the elements of the system. Endnote 4 summarizes some of the relevant background and terminology.

Early work by Brown [Brown 70] suggested that geophysical variables (timing and amount of sun, etc.) play an important role in giving time cues to circadian systems. Natural geomagnetic and geoelectrostatic fields maybe one of these entraining cues as they undergo a daily variation of about $15 \%$ in intensity. Dowse and Palmer [Dowse 69] showed that an electrostatic field can entrain the activity rhythm of mice.

Wever's is the only extensive work on the effects of low intensity fields on circadian activity of 
humans [Wever 74, Wever 79]. Human subjects (all men) lived in underground bunkers for 3 to 8 weeks at a time. One bunker was isolated from natural electromagnetic fields while one was not. Subjects living in the shielded bunker showed a tendency to have desynchronized temperature and activity rhythms, with significantly longer circadian periods than those who continued to be exposed to the natural fields. The application of a small electric field of $2.5 \mathrm{~V} / \mathrm{m}$ at $10 \mathrm{~Hz}$ reduced the internal desynchronization, while a 600 $\mathrm{V} / \mathrm{m}$ direct current (DC) failed to have any effect. Application of the low-intensity, low-frequency field in 12-hour on, 12-hour off cycles restored the circadian period to the natural value.

Work by Ehret's group at the Argonne National Laboratories monitored different rhythms of mice simultaneously while exposing them to different AC fields [Duffy 82, Ehret 80]. Their work showed that depending on the field applications protocol, phase delays were produced in activity and metabolism rhythms. However, as the field strength used in this work was very high $(130 \mathrm{kV} / \mathrm{m})$ and only one species of mice were used, the work can not be used to conclude that lower field levels affect circadian activity in rodents.

Dowse (1982) also showed that a $150 \mathrm{~V} / \mathrm{m}$ electric field at $10 \mathrm{~Hz}$ advances the phase in the locomotor activity of the fruit fly Drosophila Melanogaster under constant light conditions when the field is applied in a 12-hr on, 12-hr off pattern, perhaps taking the place of the normal LD cycle cue.

The most striking and directly demonstrated circadian effect of $60 \mathrm{~Hz}$ electric fields on animals came from the work of Wilson, Anderson and colleagues at the Battelle Pacific Northwest Laboratories. They examined the effect of continued $60 \mathrm{~Hz}$ electric field exposure on the functioning of the rat pineal gland as measured by the circadian pattern of secretion of 5-methoxytryptophol (5-MHOT, melatonin and serotonin-N-acetyl transferase (SNAT). Night levels of melatonin and SNAT in mice are generally several times higher than day levels. Field exposure in the range of intensities 1.7 to $65 \mathrm{kV} / \mathrm{m}$ depressed the melatonin rhythm, and introduced a delay in the SNAT activity rhythm. This effect, however, was not immediate on field exposure. For a $39 \mathrm{kV} / \mathrm{m}$ field, the onset of the effect appears after two weeks and before three weeks of exposure. The animal recovers from the effect on cessation of exposure in less than 3 days after initiation of exposure. There was no apparent change in the magnitude of the effect at the different field strengths.

As part of the New York State Power Lines Project, Sulzman and colleagues examined the effects of a range of electric and magnetic fields ( $O$ to $39 \mathrm{kV} / \mathrm{m}, \mathrm{O}$ to 1 gauss) on free-running periods of food and oxygen consumption of squirrel monkeys, maintained under constant light [Sulzman 86]. Although the number of monkeys used in the experiment is small $(10)$, there was a systematic increase of effect with increasing values of field intensity. The two rhythms did not show any increase in a $2.6 \mathrm{kV} / \mathrm{m}, 1 \mathrm{G}$ field; Three out of nine monkeys showed a statistically significant increase in the period of both rhythms in $\mathbf{2 6}$ $\mathrm{kV} / \mathrm{m}, 1 \mathrm{G}$; and three out of four monkeys showed an increase in a $39 \mathrm{kV} / \mathrm{m}, 1 \mathrm{G}$ field. There was no desynchronization between the two cycles measured. Both individual variation of susceptibility to the effect among the monkeys and residual effects were noted. Three of the ten monkeys involved in these experiements continued to maintain their longer period even after the removal of field exposure. Inspite of the small number of animals in the experiment, the results are reliable because the investigators have maintained several years of circadian period data on this class of monkeys and found them to be remarkably stable with little dependence on age and various kinds of treatment.

In summary, experiments on the effect of electric and magnetic fields on the circadian systems of 
man, primates and lower animals indicate a definite effect of $60 \mathrm{~Hz}$ fields on the periodicity of physiological functioning. It is not clear whether such effects are deleterious or even long-lasting. Dyschrony of the circadian system has been associated with physiological and psychological disorders ranging from altered sensitivity to drugs and toxins and internal conflicts between the timing of physiological processes to sleep, performance and other psychiatric disorders including chronic depression [Maurizi 84]. Hence it would be useful to do some careful and focused research on the potential effects of low-level $60 \mathrm{~Hz}$ fields on the circadian system in humans and animals.

\subsection{Experiments with Human Subjects}

Like many other animal species, humans can detect power-frequency electric fields through stimulation of receptors on the surface of the skin. Deno and Zaffanella [Deno 82] found the threshold of perception to depend on body posture and to vary markedly across individuals. The lowest detection thresholds for vertical electric fields were associated with stimulation of hand hair with the hand held over the head. The median threshold of perception across 136 individuals for this posture was found to be 7 $\mathrm{kV} / \mathrm{m}$. With arms at the side, the field is first perceived as stimulation of head hair. The median threshold of perception in this case was about $23 \mathrm{kV} / \mathrm{m}$. For both postures, the most sensitive individuals could detect fields smaller than $2 \mathrm{kV} / \mathrm{m}$.

In a carefully designed experiment, Graham et al. conducted a study to evaluate human perception, performance and physiology in $60 \mathrm{~Hz}$ electric and magnetic fields. The effect of fields on performance was studied through a specific battery of tests that measured effects on the $E E G^{14}$ in response to visual and auditory signals; memory; reaction time; time perception and some specific information processing abilities. Physiology was monitored by blood chemistry and other parameters such as heart rate. Subjective feelings were also elicited [Graham 871. The exposure chamber was carefully designed and built, and the study done blind with each volunteer subject serving as his own control. All 20 subjects were healthy males between 21 and 35 years of age, and were given complete explanation of the purpose of the experiment. Field strengths were $0-32 \mathrm{~A} / \mathrm{m}$ and $15 \mathrm{kV} / \mathrm{m}$ for a few hours at a time.

The perception experiments showed that while subjects differed in their sensitivity to the fields, there was no difference when the fields were presented individually or in combination. Fields increasing gradually resulted in a higher perception threshold than a sudden onset.

Most vital signs, physiological parameters, daily life activities and moods were not affected by the field strengths presented. Also not affected were simple reaction time, memory span, fatigue and ability to make decisions. Some changes were detected in heart beat interval and on tests demanding specific EEG activity but the variations were within normal ranges for these parameters.

Followup studies of the observed heartbeat changes showed some definite effects in heartbeat rate and performance. Two groups of fifteen subjects each were exposed to different exposure conditions. One group was subjected to field exposure for 6 hours in alternating 45-minute periods of field on and off. The second group was exposed to a fast, intermittent exposure pattern of 15 seconds of switching on and off for periods of 45 minutes followed by 45 minutes of fields off. While preliminary, the results suggest

\footnotetext{
${ }^{14}$ The EEG or electroencephalogram, is a record of the variations in electric potential recorded from the brain and is indicative of the electrical activity in the brain. EEG patterns depend upon diverse factors such as age, sleep state, blood glucose, and levels of brain hormones.
} 
that alertness and reaction time were notably affected in the intermittent exposure group and there is an individual variation in individual susceptibility to fields. [Graham 88] 


\section{Comparing Laboratory and Human Exposures}

Studies of the bioeffects of power-frequency fields described in the preceding sections, involve many different subjects, exposure systems, and exposure regimens. Animal studies have examined field effects on rats, mice, miniature swine, cows, guinea pigs, and chicken eggs. in vitro studies have employed perfused nervous system tissue, cell cultures, and entire organs (e.g. heart, brain). This section describes electric and magnetic induction in these experimental systems and presents a comparison of the exposures in several specific experiments to the exposures in the set of common human exposure situations described in Section 2.6.

\subsection{Laboratory Animals - Electric Induction}

The factors that most affect electric induction are body shape, the orientation of the body relative to the field, and body grounding. The charge induced on the surface of the body is independent of both body size and the conductivity of body tissue. The conductivities of various body tissues do, however, affect the specific paths that induced currents take through the body.

Rough estimates of body-averaged quantities can be obtained from simple theoretical models such as those by Barnes and colleagues [Barnes 67] which approximate the body as an elongated sphere. Table 5-1 gives the body-average internal electric fields and current densities that such models predict for a number of different species under several exposure conditions.

The data in Table 2-9 show clearly that electrically-induced fields and currents are most intense when the applied field is parallel to the long axis of the body. For such a condition, the data also show that induced fields are greatest for the most elongated species (i.e. humans). Finally, these calculations indicate that body-average exposure intensity is more intense when the body is grounded than when it is ungrounded.

\subsection{Laboratory Animals - Magnetic Induction}

By Faraday's law, the intensity of the current induced at any point in the body by a spatially uniform magnetic field is proportional to the rate of change of flux through all closed current paths that include that point. This means that both magnetically-induced current density and magnetically-induced electric fields will tend to zero near the body center and reach maximum values at those areas of body surface that lie most parallel to the applied magnetic field. Both peak and volume-averaged magnetically-induced electric field and current density tend to scale with body radius.

Estimates of magnetically-induced electric fields and currents come primarily from theoretical models that treat the body as a homogeneous sphere, spheroid, or ellipsoid [Kaune 86, Spiegel 76, Spiegel 77]. A limited number of measurements have been made of magnetic induction in homogeneous human models [Guy 76]. These measurements demonstrate the presence of local induced fields that are several times stronger than volume-average values.

Using the same approach adopted above for comparing body-average electric induction in animals and humans, we have computed the magnetically-induced volume-averaged current density and the volume-averaged electric field induced in a set a spheroids with dimensions chosen to approximate the shape of several species including man. Our calculations are based on an analysis by Kaune that can be used to estimate the electric field at any point within a homogeneous spheroid exposed to a uniform 
Table 5-1: Electric induction in various species by a $60 \mathrm{~Hz}$ uniform electric field of $1 \mathrm{kV} / \mathrm{m}$. $E_{i}$ is the average magnitude of internal electric field, and $\mathrm{J}_{i}$ is the volume-averaged current density. Estimates are presented for the field both parallel and perpendicular to the long axis of the body and for both the grounded and ungrounded condition. $a / b$ is the ratio of the lengths of the long and short axes of the elongated sphere used to approximate body shape. Induced electric field is given in millivolts per meter. Current density is given in nanoamps per square centimeter. Adapted from [Barnes 67].

\begin{tabular}{llllll}
\hline Specie & a/b & Grounding & $\begin{array}{l}\text { Field orientation } \\
\text { relative to long axis }\end{array}$ & $\begin{array}{l}E_{i} \\
(\mathrm{mV} / \mathrm{m})\end{array}$ & $\begin{array}{l}\mathrm{J}_{i} \\
\left(\mathrm{nA} / \mathrm{cm}^{2}\right)\end{array}$ \\
\hline Human & 5.5 & Grounded & Parallel & 1.0 & 20. \\
Human & 5.5 & Ungrounded & Parallel & .35 & 7. \\
Human & 5.5 & Ungrounded & Perpendicular & .035 & .7 \\
cow & 4.5 & Grounded & Perpendicular & .04 & .8 \\
cow & 4.5 & Ungrounded & Parallel & .25 & 5. \\
cow & 4.5 & Ungrounded & Perpendicular & .035 & .7 \\
Swine & 3.0 & Grounded & Perpendicular & .045 & .9 \\
Swine & 3.0 & Ungrounded & Parallel & .15 & 3.0 \\
Swine & 3.0 & Ungrounded & Perpendicular & .035 & .7 \\
Mouse/rat & 2.7 & Grounded & Perpendicular & .045 & .9 \\
Mouse/rat & 2.7 & Ungrounded & Parallel & .13 & 2.5 \\
Mouse/rat & 2.7 & Ungrounded & Perpendicular & .04 & .8 \\
Chick egg & 1.0 & Ungrounded & Either & .02 & .4 \\
yolk* & & & & & \\
\hline
\end{tabular}

. Field in yolk estimated using concentric sphere model with conductivities

of $2.5 \mathrm{~S} / \mathrm{m}^{15}$ and $7.2 \mathrm{~S} / \mathrm{m}$ for yolk and albumin, respectively [Tinga 73 ].

magnetic field [Kaune 86]. Magnetic induction was estimated for applied fields both parallel and perpendicular to the long axis of the body. The results of these calculations are presented in Table 5-2.

This analysis shows that magnetically-induced body currents are much larger for large species than for small ones. In comparing the magnetic fields used in lab animal experiments to the fields that humans commonly encounter, one may want to adjust laboratory exposure intensities to account for these differences in magnetic induction. Another conclusion one can draw from Table 5-2 is that induced currents are slightly larger when the field is applied perpendicular to the long axis of the body. This is a consequence of Faraday's Law, which says that induced currents are proportional to the area enclosed by the current loop.

\footnotetext{
${ }^{15} \mathrm{~S} / \mathrm{m}$ or, Siemens per meter is the unit for specific conductivity. The Siemen (S) is the same magnitude as the formerly used (ohm)-I’
} 
Table 5-2: Magnetic induction in a homogeneous elongated sphere by a $60 \mathrm{~Hz}$ uniform magnetic field of 1 gauss. The long axis of the spheroid, $a$, and the ratio, $a / b$, of the lengths of the long and short axes are chosen to approximate the dimensions of various species. $E_{i}$ is the average magnitude of the magnetically-induced electric field inside the body (in millivolts per meter) and $\mathrm{J}_{i}$ is the average magnitude of the magnetically-induced current density (in nanoamps per square centimeter). Based on analysis by Kaune [Kaune 86].

\begin{tabular}{llllll} 
Specie & $\begin{array}{l}a \\
(\mathrm{~m})\end{array}$ & $\mathrm{a} / \mathrm{b}$ & $\begin{array}{l}\text { Field orientation rel- } \\
\text { ative to long axis }\end{array}$ & $\begin{array}{l}\mathrm{E}_{\mathrm{i}} \\
(\mathrm{mV} / \mathrm{m})\end{array}$ & $\begin{array}{l}\mathrm{J}_{\mathrm{i}}^{*} \\
\left(\mathrm{nA} / \mathrm{cm}^{2}\right),\end{array}$ \\
\hline Human & 1.7 & 5.5 & Parallel & 3.4 & 68. \\
Human & 1.7 & 5.5 & Perpendicular & 4.4 & 88. \\
cow & 2.5 & 4.5 & Parallel & 6.2 & 120. \\
cow & 2.5 & 4.5 & Perpendicular & 8.0 & 160. \\
Swine & 0.9 & 3.0 & Parallel & 3.3 & 66. \\
Swine & 0.9 & 3.0 & Perpendicular & 4.3 & 6.6 \\
Mouse & 0.08 & 2.7 & Parallel & 0.33 & 8.4 \\
Mouse & 0.08 & 2.7 & Perpendicular & 0.42 & 14. \\
Rat & 0.17 & 2.7 & Parallel & 0.70 & 18. \\
Rat & 0.17 & 2.7 & Perpendicular & 0.89 & 200. \\
Chick Egg & 0.025 & 1.0 & Either & 0.28 & \\
(Yolk) & & & & & \\
\hline
\end{tabular}

-- Assumed conductivity is .2 S/m except $7.2 \mathrm{~S} / \mathrm{m}$ for egg yolk [Schwan 57 , Tinga 73 ].

\subsection{In Vitro Experiments}

This section describes the tissue currents associated with some of the in vitro experiments described in Section 3. The preparations used in these experiments include chick brains, cultured blood, cancer, and bone cells, and adrenal tissue. Exposure means include placing the preparation within magnetic field coils, exposing cells to special radio frequency fields in large wave guides, and by placing electrodes directly into their growth medium of the cells.

\subsubsection{Pulsed Magnetic Field Exposures}

The bioeffects literature contains many references to both in vivo and in vitro experiments that involve exposures to pulsed magnetic fields generated by Helmholtz coils. Many of these experiments involve pulses with rise times of about 100 microseconds and peak magnetic fields of about 10 Gauss. Such systems are capable of inducing peak electric fields of about $1 \mathrm{mV} / \mathrm{cm}$ in tissues of $1 \mathrm{~cm}$ diameter and proportionately larger electric fields in tissues of larger dimension. For typical tissue conductivities of .2 Siemens per meter, this corresponds to a current density of $200 \mathrm{nWcm}^{2}$. Induced fields and currents in tissues with dimensions greater than $1 \mathrm{~cm}$ are proportionately larger. 


\subsubsection{ELF-Modulated Radiofrequency Exposures}

There are a number of experimental studies (those by Adey [Adey 82], Bawin [Bawin 75], Blackman [Blackman 85a], and Lyle [Lyle 83] being notable examples) that have demonstrated bioeffects of exposures to radiofrequency fields (100-1000 Mhz) that are amplitude-modulated at ELF frequencies $(0-100 \mathrm{~Hz})$. Amplitude modulation, as applied here, means that the intensity of the radiofrequency field is varied sinusoidally at ELF frequencies. Because electric fields induced in tissue are proportional to frequency, radiofrequency fields couple much more strongly to tissues than do ELF fields. The radiofrequency fields in the experiments listed above induce radiofrequency fields in the exposed tissue of $1-10 \mathrm{~V} / \mathrm{m}$.

Given evidence suggesting that the mechanisms by which fields interact with cells are nonlinear, some scientists have proposed that cells may be capable of "demodulating" amplitude-modulated fields. That is, cells may be able to extract the ELF component of the high frequency field. If this is true, the resulting ELF fields in tissue would be orders of magnitude larger than the ELF fields induced in humans by the power-frequency fields of power lines and appliances.

\subsubsection{Calcium Efflux from Chick Brains}

Blackman and colleagues have exposed chick brain halves to ELF electric fields to study effects on calcium efflux [Blackman 85a]. Brain halves were placed in test tubes along with enough saline solution to just cover the brain tissue. The test tubes were placed in an exposure chamber capable of creating ELF fields in air of $10-20 \mathrm{~V} / \mathrm{m}$. Blackman et. al. estimate that the electric field induced in the exposed brain tissue is about $10^{-7} \mathrm{~V} / \mathrm{m}$ at $45 \mathrm{~Hz}$ and proportionately larger or smaller at other frequencies. This corresponds to an induced current density of $.01 \mathrm{nA} / \mathrm{cm}^{2}$. These intensities are comparable to the electric fields and currents induced in the body of a person standing in a vertical $60 \mathrm{~Hz}$ electric field of about 1 $\mathrm{V} / \mathrm{m}$.

\subsection{Comparison of Exposures in Bioeffects Studies to Common Human Exposure Situations}

Comparisons between the exposure conditions of several of the bioeffects studies described in Sections 4-3 and the exposures that people commonly encounter can, of course, be made along many different exposure dimensions. Figure 5-1 compares common human exposure situations with experimental exposures along just one of these dimensions, the volume-averaged, time-peak current density. The experimental exposures listed in Figure 5-1 span the range of common human exposure situations. This same observation is likely to hold for many other measures of exposure that one might choose to examine. In other words, biological effects of power-frequency fields have been demonstrated across the range of exposure conditions that people commonly encounter. Effects are not limited to situations involving only very intense or otherwise unusual fields. 


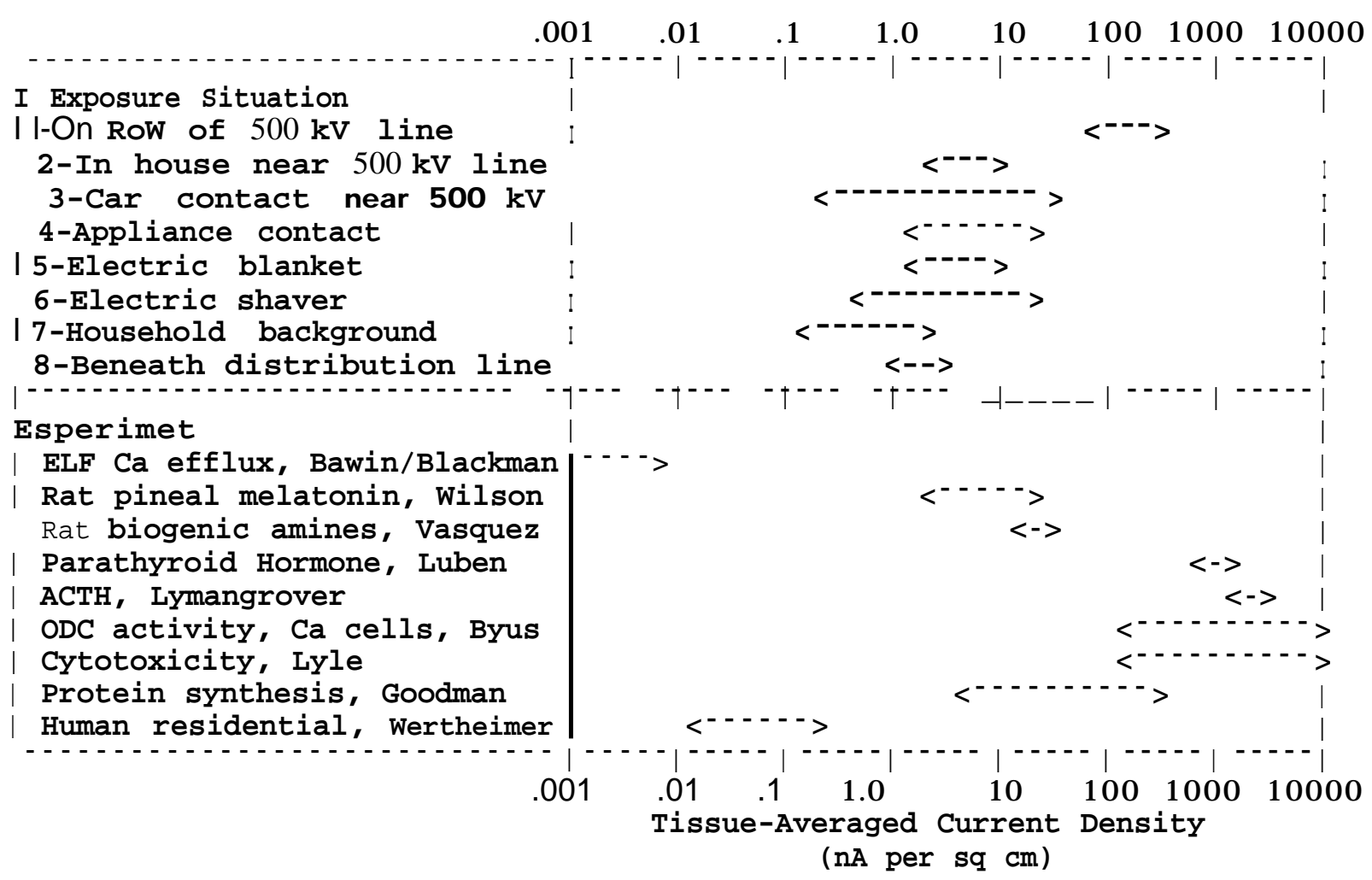

Figure 5-1: Average current density associated with eight common human exposure situations compared to average current densities in a number of laboratory experiments. Adapted from [Florig 87b]. 


\section{Cancer and Electromagnetic Fields: Epidemiological Studies}

Cancer incidence in children and cancer as related to occupational ELF field exposure are the two areas that have received the most attention in the context of public health consequences of exposure to ELF fields. This concern with cancer arose as a result of an epidemiological study and has increased as several additional epidemiological studies have examined the association between ELF field exposure and cancer. The suggestion is not that $60 \mathrm{~Hz}$ fields initate cancer but rather that exposure to fields may serve to promote cancer once it is initiated by other causes (See endnote on promotion).

As biologic knowledge about an epidemiologic hypothesis is often missing or minimal, causal inference is hard to make. At the same time, public health problems or public perception of potential health problems may cause an action to be taken by decision-makers, despite imperfect knowledge about cause. Hill [Hill 65] developed a set of criteria by which to sort out causal from noncausal associations. These are :

1. Strength of association : that is, magnitude of the ratio of incidence rates in exposed populations to that in non-exposed, for example, the relationship between higher levels of smoking and lung cancer.

2. Consistency : Observation of same association in different populations under different criteria, for example, increased lung cancer incidence in smokers of all age groups, and races.

3. Specificity : single rather than multiple effects, as for example, when all the females exposed to DES prenatally exhibited the incidence of a particular type of cancer.

4. Temporality : Cause should precede effect in time.

5. Biologic gradient: That is, a dose-response relation or a definite mathematical relation between the amount of the exposure to an environmental agent and the incidence of the effect.

6. Biologic plausibility and coherence : A potential mechanism and an absence of conflict between the association and with what is known about the history and biology of the disease.

7. Experimental evidence : Particularly, animal data that points to the same association. This may not always hold. For example, some substances such as DES may not be carcinogenic in animals.

8. Analogy : If there is another agent that is analogous to the agent under consideration, and its biologic effect is well- (or better) known, we can look for analogous modes of action and effects.

In light of our discussion of "dose", and nonlinear effects in Sections 3, criterion 5 above needs to be redefined and elaborated before it can be applied to ELF field exposure. Also, there is no analogous agent of the sort mentioned in criterion 8 . Beyond this, we believe that the remaining criteria are directly applicable to epidemiological studies of ELF fields. At most, these considerations make the point that a further examination of the question through carefully designed, focused epidemiologic) studies are necessary in addition to laboratory experiments to understand the science before any firm conclusion can be drawn. 


\subsection{Childhood Cancer and ELF fields}

Five completed epidemiological studies have addressed the question of association between exposure to ELF fields and cancer in children. These are all case-control studies. The Wertheimer and Leeper study [Wertheimer 79] in Denver first raised the question. The authors noted an association between childhood cancer and homes they classified as located near "high current configuration" distribution lines which were likely to produce stronger than average magnetic fields. The authors studied cases of children of age less than 19 years who died of cancer between 1950 and 1973, and who also had a Colorado birth certificate and resided in the area during most of their lives. The controls were children whose birth certificates placed "next" to the case children in the public birth files organized by birth month and county, except in the case when the "next" case was a sibling of a case child. 344 cases and 344 controls were examined.

After performing a series of measurements of magnetic fields to develop a simple classification scheme, the authors estimated the comparative magnitude of the magnetic field in the home by the surrogate measure of wiring configurations. Homes were classified as "HCC" and "LCC" ( for high and low current configurations). A house was classified as "HCC" if one of the following conditions was satisfied : (1) it was close to a large gauge or several (6 or more) thin primary current-carrying wires from distribution transformers; (2) it was close to 3 or more thin primaries or high voltage (50kV to $230 \mathrm{kV})$ lines, (3) it was less that 15 meters of wires coming directly from a transformer. A series of field measurements was performed to confirm these classifications.

The results showed that children exposed to a HCC configuration residential environment had a 1.6- to 2.2 -fold higher incidence of cancer than controls. Background incidence rate for childhood leukemia in children between the ages $O$ to 14 years is about 10 in 100,000 per year [Greenberg 85]. If the children exposed to HCC configuration have a risk of 1.6 to 2.2 (or, about 2), relative to this background rate, this means that the risk of children exposed to $\mathrm{HCC}$ is double the base rate, or 20 in 100,000 per year. The study ruled out socioeconomic class, family pattern and traffic congestion near homes as possible confounders but did not deal with other possible factors that could cause confounding or bias. The results have been widely debated and criticized since their publication [Cole 87, Savitz 87b]. Among the limits of the study are : biases due to survival aspects introduced by studying only the cancer deaths rather than all cancers diagnosed; bias introduced by residential mobility; validity of the exposure measure; and, failure to account for other confounders.

Four studies have been done since the Wertheimer and Leeper study. Two of them found no association between leukemia and estimated exposure to magnetic fields. These two studies were conducted in Rhode Island [Fulton 80] and in Yorkshire, England [Myers 85]. The Rhode Island study included 119 childhood leukemia incidence cases and 240 controls, and was similar in design to the Wertheimer and Leeper study. The study in Yorkshire looked at all children diagnosed with cancer during 1970-79, a total of 376 cases and 591 controls. Residence classification was first made in terms of proximity to overhead power lines. Among those of the homes within $100 \mathrm{~m}$ of the power lines, magnetic fields were estimated. There was no relationship observed between cancer incidence and either exposure metric.

Two other studies found positive results. The first of these was done in Sweden [Tomenius 86]. All 716 children diagnosed with cancer, and born and raised in Stockholm County during 1958-73 were included in the study. Tomenius found that there were more electrical constructions near dwellings of 
children who had cancer than of controls.. A single measurement of magnetic field near the front door was also taken for each house. There was no difference between the average value of magnetic fields at the doors of homes that were near electrical constructions or 200-kV lines and those that were not. However, magnetic fields at the door of homes within $150 \mathrm{~m}$ of $200 \mathrm{kV}$ lines were higher than at the houses that did not have such lines close by. It was found that homes of cancer cases were twice as likely to have a front-door measurement of $3 \mathrm{mG}$ or above than those of controls. The strongest odds ratio of 3.7 was noted for nervous system tumors.

The latest and by far, the most thorough and complete study is that by Savitz et al. [Savitz 87a, Savitz 88]. The study was designed to be similar to that of Wertheimer and Leeper, but with particular attention paid to several factors that were weaknesses in the former study. These were: a complete set of incident cases because of the use of the registry; use of a general population control group; expanded and detailed exposure measurement; and, measurement of possible confounding factors.

The Savitz study involved children from the Denver Standard Metropolitan Statistical Area of age 15 or less diagnosed as having cancer during the period 1976 to 1983, a period distinct from that of the Wertheimer and Leeper Study, which covered 1950 to 1973. Both wire coding and actual measurement of fields in the house were used to characterize the residential field environment. Detailed questionnaires elicited information about socioeconomic and other possible confounding factors such as smoking. An analysis of the total childhood cancers occurring in the Denver area children was also done and showed that Denver area children show the same overall risk as those in the National Cancer Institute's Surveillance, Epidemiology, and End Results (SEER) Program.

Electric and magnetic fields data at three different times in a child's chronology were selected for measurements and exposure assessments: the fields in the residence of birth to reflect early-life exposure; those at two years before the diagnosis to examine an effect of the field in promoting cancer; and, those at diagnosis. For each of these three times four types of field measurements or estimates were made : (1) magnetic field in the house when electric power consumption was low, (2) magnetic field in the house when electric power consumption was high, (3) electric field in the house when electric power consumption was high, and (4) the distribution system wire code classification using the system developed by Wertheimer and Leeper. An array of field measurements - fields in different rooms, variation over the day, etc. - was done. In fact, this part of the Denver study, conducted by Barnes and Wachtel [Barnes 87] has become a prime source in understanding fields in the home (see Section 2.2). For each power range, measured levels of magnetic fields were classified into four ranges : less than $0.65 \mathrm{mG}$, 0.66 to $0.99 \mathrm{mG}, 1.00$ to $2.49 \mathrm{mG}$ and $2.50+\mathrm{mG}$. Wire coding was used as a proxy for the long-term magnetic field level after it was calibrated using measurements.

The study also assessed other measures of potential field exposures such as electric heat and hot water use, use of water beds, heating pads and electric blankets by the children and by mothers during pregnancy and the total number of electrical appliances in the house. The results showed that the highest field $(2.5+\mathrm{mG})$ group in each power category showed a increased risk of about 20 to $60 \%$ above the controls for all cancers. The values for risk correlated with fields at two years before exposure and at time of diagnosis were similar. The odds ratio in this study was computed by taking the ratio of cancer incidence in each field group to the lowest $(<0.65 \mathrm{mG})$ group. The Odds Ratio $(\mathrm{OR})$ for a group is a measure of how much more likely a child in that exposure group is to have cancer compared to a child in the lowest exposure group. The general results are summarized below: 
Table 6-1. - Methodology and Results of Epidemiologic Studies of Childhood Cancer and Electromagnetic Field Exposure (Savitz 87a)

\begin{tabular}{|c|c|c|c|c|}
\hline & Wertheimer \& Leeper (1979) & Fulton et al. (1980) & Myers et al. (1985) & Tomenius (1986) \\
\hline $\begin{array}{l}\text { Geographic } \\
\text { source }\end{array}$ & Colorado & Rhode Island & $\begin{array}{l}\text { Yorkshire (England) } \\
\text { Health District }\end{array}$ & Stockholm County \\
\hline \multicolumn{5}{|c|}{ Case group: } \\
\hline Time period & Deceased 1950-73 & Onset $1964-78$ & Diagnosed 1970-79 & Registered 1958-73 \\
\hline Diseases & All cancers & Leukemias & All cancers & All tumors \\
\hline Age range & O to 18 & 0 to 20 & 0 to 14 & 0 to 18 \\
\hline $\begin{array}{l}\text { Other } \\
\text { criteria }\end{array}$ & $\begin{array}{l}\text { Colorado birth certificates; } \\
\text { resided in Denver area, } \\
\text { 1946-73 }\end{array}$ & $\begin{array}{l}\text { Identified at Rhode Island } \\
\text { Hospital: Residences up to } \\
8 \text { years before diagnosis }\end{array}$ & - & $\begin{array}{l}\text { Born \& diagnosed in } \\
\text { Stockholm County }\end{array}$ \\
\hline \multicolumn{5}{|c|}{ Control group: } \\
\hline Source & Birth certificates & Birth certificates & Birth certificates & Birth certificates \\
\hline Matching & Year of birth; some by county & Year of birth & $\begin{array}{l}\text { Time of birth, near case's } \\
\text { birth address }\end{array}$ & Age, sex, church district \\
\hline $\begin{array}{l}\text { Other } \\
\text { criteria }\end{array}$ & $\begin{array}{l}\text { Subsets formed based on } \\
\text { residence information }\end{array}$ & $\begin{array}{l}\text { Only birth addresses } \\
\text { considered }\end{array}$ & $\begin{array}{l}\text { Only birth addresses } \\
\text { considered }\end{array}$ & $\begin{array}{l}\text { Birth \& "diagnosis" address in } \\
\text { Stockholm }\end{array}$ \\
\hline $\begin{array}{l}\text { Exposure: } \\
\text { Definition }\end{array}$ & $\begin{array}{l}\text { Wiring configurations (wire } \\
\text { type, gauge, number, } \\
\text { proximity to home) }\end{array}$ & $\begin{array}{l}\text { Estimated exposure from } \\
\text { Colorado measurements, } \\
\text { divided into quartiles }\end{array}$ & $\begin{array}{l}\text { Calculated magnetic fields } \\
\text { from overhead lines }\end{array}$ & $\begin{array}{l}\text { Electrical constructions within } \\
150 \mathrm{~m} \text { including } 200-\mathrm{kV} \text { lines; } \\
50-\mathrm{Hz} \text { magnetic fields near } \\
\text { door }\end{array}$ \\
\hline Range & Up to $35 \mathrm{mG}$ & N.A. & 0.002 to $16.8 \mathrm{mG}$ & 0.004 to $19 \mathrm{mG}$ \\
\hline $\begin{array}{l}\text { Potential } \\
\text { confounders }\end{array}$ & $\begin{array}{l}\text { Age of onset; sex; urban- } \\
\text { rs suburban residence; } \\
\text { socioeconomic class; } \\
\text { maternal age; birth order; } \\
\text { traffic density }\end{array}$ & $\begin{array}{l}\text { Year of birth; father's } \\
\text { socioeconomic level; age of } \\
\text { onset }\end{array}$ & Age & Age, sex, church district \\
\hline Results & $\begin{array}{l}\text { Positive association between } \\
\text { high-current configurations } \\
\text { and cancer; dose-response } \\
\text { gradient; consistent across } \\
\text { cancers }\end{array}$ & $\begin{array}{l}\text { No association observed } \\
\text { between imputed exposure } \\
\text { \& leukemia }\end{array}$ & $\begin{array}{l}\text { No consistent tendency for } \\
\text { higher exposures among } \\
\text { cases }\end{array}$ & $\begin{array}{l}\text { More electrical constructions } \\
\text { within } 150 \mathrm{~m} \text { of case homes; } \\
\text { more case homes }>3 \mathrm{mG}\end{array}$ \\
\hline
\end{tabular}


1. A $\mathbf{3 0} \%$ increase in risk (Odds ratio $=1.31$ ) for all cancers was observed at fields in the category $2.50 \mathrm{mG}$ + but none was observed in the two field ranges below $(0.66$ to $0.99 \mathrm{mG}$ and 1.00 to $2.49 \mathrm{mG}$ ). The odds ratio did not systematically increase or decrease with the field magnitudes. That is, the higher the field ranges did not always give a higher cancer risk,

2. Cancer subgroups were analyzed under the categories, leukemia, lymphoma, brain tumors, soft tumors and "other cancers". All the categories except leukemia showed odds ratios 1.3 to 1.6 at high $(2.5 \mathrm{mG}+)$ field exposures only, Leukemia showed an odds ratio of 2.11 for the highest field class and 1.23 for the next one below.

3. The risk of cancer was not associated with measured magnetic field values at residence of birth.

4. For the one category (high-power) of electric field ranges analyzed, no association of the electric field categories with the odds ratio for cancers was found. That is, higher electric fields did not show higher risk of cancer.

5. Comparing odds ratio $\mathrm{HCC} / \mathrm{LCC}$ of wiring configurations, odds ratios between 1.5 and 2.1 was found for all cancers with the highest 2.10 occurring for leukemia.

6. The results on the relationship of childhood cancer to use of appliances, electric blanket and waterbed, and electric heat are mixed but suggestive of a few trends. It must be remembered that the number of cases in the sample is small :

a. Electric heat in residence and electric hot water: Lymphoma and cancers other than leukemia, brain, and soft tissue had odds ratio above 3.5, associated with electric heat in residences at birth, 2 years before diagnosis and at diagnosis. Leukemia showed elevated odds ratio for electric heat in residences two years before diagnosis, but not for electric heat for residences at birth and at diagnosis. Electric hot water in the residence showed no association with any of the cancers.

b. Electric blanket, heated waterbeds, heating pad and total number of electrical appliances used by mother during pregnancy: Although electric blanket and water beds are known sources of high fields for in utero exposure, Savitz found no indication of increased cancer risk associated with such exposure. Heating pad use was associated with brain and soft tissue tumor cases. High appliance use was associated with soft tissue tumors, this relationship showing a monotonic increase in number of cases with increased appliance use from an odds ratio of 1.72 for 3-4 appliance use to 3.98 for 7 or more appliances compared to low (0-2) appliance use.

c. Childhood appliance exposure:

Electric blanket and isolette exposures were associated with increased risk of all cancers especially of the brain and soft tissue for isolette exposure.

Cole (1987) points out that the hypothesis of ELF magnetic fields as promoters or as growth enhancers would lead one to expect the average age of the highly exposed children in the childhood cancer studies would be lower than that for the control group. This, however, does not seem to be the case. Wertheimer has, however, argued that this lack of early appearance of cancer may be an artifact arising from the fact that if the promotion is pre- or perinatal, the fetal survival rate might have been affected and one might be seeing only a fraction of the affected children surviving to manifest the clinical cancer stage. 


\subsection{Residential Exposure and Adult Cancer}

Three studies have examined the association between adult cancer and exposure to ELF fields from non-occupational sources, two in the U.S. examining residential exposures and one in England examining the proximity of residences to electrical transmission facilities.

Wertheimer and Leeper were also the first to report an association between adult cancers and residential wiring configurations in 1982 [Wertheimer 82]. They used a methodology parallel to the childhood cancer study described above. Four categories of wiring configurations were used to characterize residences in which the subject had lived for periods from three to ten years prior to the diagnosis of cancer. The authors reported an association between cancers of the nervous system, uterus and breast with a systematically increasing risk for higher current configurations. The strongest association was found for those subjects who had been in the coded residence for three to six years right before diagnosis and no association was found when the subjects had left the residence three years or more prior to it. Leukemia was not considered. The study also did not look for confounding factors and exposure determination was not done blind. When a study is not done blind, a bias on the authors' part to find an association might lead to a false positive result. Cole [Cole 87] notes that there is a "remarkable consistency" about the demonstrated relationship in the study. He remarks such a consistency is atypical of epidemiological studies even when a causal relationship actually exists, because of the large uncertainties that accompanies the data.

Stevens and coworkers [Stevens 87] at Battelle Pacific Northwest National Laboratories carried out a case-control study under the New York State Power Lines Project. Acute nonlymphocytic leukemia (ANLL) incidence was chosen as the effect to study because several of the occupational studies had indicated an association of ANLL incidence with occupations involving ELF field exposures. Wiring configurations and residential field measurements over 24 hour periods were used to characterize residential exposures. The cases of ANLL used were those registered at the Cancer Surveillance System at the Fred Hutchinson Cancer Research Center in Seattle, Washington. Cases of ANLL occurring in three counties in persons between 20 and 79 years of age and diagnosed in the period 1981 to 1984 were used, compared with controls generated by random-digit dialing. The study found no association between ANLL incidence and residential fields.

McDowall [McDowall 83] identified a cohort of people living in the vicinity of transmission lines and power substations in East Anglia in England and did a followup mortality study from 1971 to December 1983. He found no increased leukemia mortality incidence for this population compared to national or regional mortality rates.

These three studies were varied in concept and have several problems, such as confounding variables and uncertainties in the amount of field exposure, and do not provide enough evidence to judge the possibility of an association between residential field exposure and adult cancer.

\subsection{Occupational Exposure and Cancer}

We now discuss the studies of occupational exposure to ELF fields and the association with cancer in three categories, those examining associations with Leukemia, with brain cancer and with all cancers. Savitz and Cane [Savitz 87b] have reviewed the leukemia studies.

About twenty studies have looked for an association between cancer, particularly leukemia and 
brain cancer and occupational exposure to ELF fields. Studies have been done using electrical worker populations or ham radio operators in the U. S., England, Sweden and New Zealand. All except 5 Swedish studies have been proportionate mortality or morbidity studies or case-control studies. The five Swedish studies are retrospective follow-up studies which tend to be more reliable than proportionate mortality studies. The results of all studies taken together indicate a small positive association or no association.

\subsubsection{Leukemia}

Four occupational studies of U.S. male populations looked at leukemia deaths of "electrical" occupations as the end-point and calculated proportional mortality ratios [Milham 82, Wright 82, Cane 83, Milham 85]. Additionally, two U.S. studies of causes of cancer deaths [Peterson 80, Dubrow 80] yielding data for the association between leukemia and occupational ELF exposure have been examined by Savitz and Cane in their review article. Two studies from England and one from New Zealand have examined leukemia incidence in electrical workers. Leukemia was also examined in three retrospective follow-up occupational exposure studies done in Sweden [Olin 85, Barregard 85, Tornqvist 86]. Some of these studies also sorted out the incidence of or death rates from acute leukemias. Table 3 from the paper by Savitz and Cane [Savitz 87b] presents the results of their calculations combining data from eleven of the studies to obtain relative risk (RR) associated with specific electrical occupations, and the relevant confidence levels.

In Table 6-2, the highest RR's -- for electrical equipment assemblers and aluminum workers have large uncertainties associated with them. The third group -- telegraph, radio and radar operators -- for whom the largest data set was available, show a consistently increased risk ( $R R=1.8)$. The other sets showing RR's above 1.5 consisted of relatively small numbers of cases. There is a relative enhancement for acute leukemia in the top four groups.

Cole's review of epidemiologic studies include two additional case-control studies of the association between occupational ELF field exposure and leukemia [Cole 87]. One of these is a study of Swedish acute myelogenous leukemia cases among electrical occupations [Stern 86] which found a RR of 3.8. The second is an analysis of nuclear shipyard workers data, looking for association between leukemia cases and electrical jobs which found a RR of 2.3 for myeloid leukemia and 6 for lymphatic leukemia.

Based on the set of studies discussed above, it is fair to say that there is an indication that occupational exposure in "electrical occupations" is associated with enhanced leukemia risk. Remember that "associated" means "occurs together with"; it does not imply a causative link. The job classifications do not clearly indicate the actual occupational exposure to fields. No confounding variables and household and other exposures have been taken into consideration in these studies. Savitz's table above also involves combining data from U.S. and non-U.S. populations which might add to the spectrum of confounding, although the effect of such addition would be to decrease rather than increase the risk numbers if there were no real association at all.

Collectively the studies do not provide good evidence that ELF field exposure increases the risk of leukemia. At the same time the evidence precludes categorical statements that no such risk exists. 
Table 6-2: Comparison of Occupational Studies of ELF exposure and leukemia [Savkz 87b].

Total Leukemias, Acute Leukemias, and Acute Myelogenous Leukemias in Electrical Occupations*

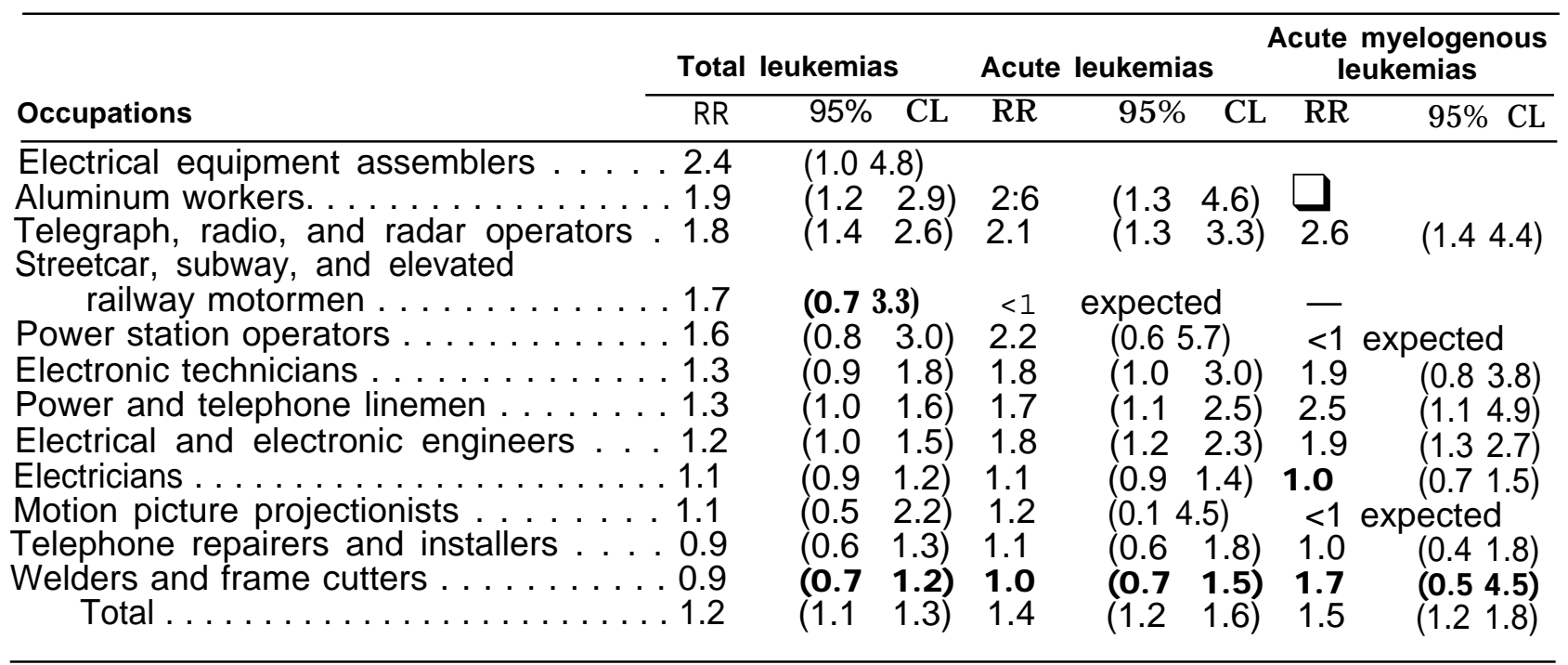

Abbreviations used are: RR, reliative risk; CL, confidence limits.

\subsubsection{Brain and Central Nervous System (CNS) Tumors}

The association between brain and CNS tumors and ELF field exposure related to occupation has been examined in about 10 studies, some of which are general cancer studies referred to in the above section on leukemia [Milham 85, Peterson 80 , Olin 85]. Other studies looked specifically at brain and CNS tumor mortality risk in men with electrical jobs [Lin 85, Thomas 87] or at the association between cancer and electrical jobs or all jobs and sorted out the types of cancers [McLaughlin 87, Preston 82, Thomas 87, Vagero 83, Vagero 85].

Brain cancer is rare (1\% of all cancer incidence, implying a risk of 5 in 100,000$)$ in adults, peaking at about 60 years of age with a histology different from that seen in children in whom brain cancer is the second high-risk cancer (20\% of the cancer occurring in the age group 0-8 years) peaking at about 5 years [Gold 79, Russell 71]. The small numbers of occurrence of brain cancers in adults poses a data problem in establishing causal association. The brain is also a favored site for metastasis and hence

\footnotetext{
${ }^{16}$ Metastasis refers to secondary growth of cancer that spreads from a primary site.
} 
cases counted as primary brain cancer may actually be secondaries spreading from a different organ in which the cancer was actually initiated.

Separate studies by Lin and Milham in 1985 were the first to suggest an association between brain/CNS tumors and occupational exposure to ELF fields [Lin 85, Milham 85]. Lin's case-control study is exceptional in the set of all occupational studies because he sought to correlate the brain tumor risk (RR) derived from death certificate data with the likelihood of exposure on the job. Based on occupational data from Maryland for 1974 to 1984, Lin obtained a relative risk of 2.2 for jobs with "definite" exposure 2.0 for those with "probable" exposure, 1.4 for "possible" exposure, and 1.0 for "no" exposure compared to age matched deed controls. His results indicate statistical significance, consistency, and an association between higher doses and higher risk of brain tumor.

Thomas and colleagues at the National Cancer Institute used death certificate data from northern New Jersey, Philadelphia and southern Louisiana to do a case-control study of the association between brain tumor mortality risk and electrical jobs, and found that data when all electrical jobs were combined showed an excess risk for astrocytic tumors $(R R=3.9)$. But his group included engineers, teachers, technicians, repairers and assemblers. When the data for electric manufacture and repair workers were separated, this high risk remained $(\mathrm{RR}=4.9 ; 95 \% \mathrm{Cl}=1.9,13.2)$ and increased tenfold among those employed for 20 or more years. Data for electrical tradesman (electrician; power and telephone lineman) showed no statistically significant increased risk. The authors conclude that although ELF field exposure cannot be definitely or uniquely identified as an causative agent, some aspect of the manufacturing and repair jobs mentioned above does place the workers at increased risk of brain tumors.

All the studies of Swedish workers [Vagero 83, Vagero 85, Olin 85, Barregard 85, McLaughlin 87] saw no excess risk for CNS or brain cancer in electrical occupations.

Lin has also completed a study of employees of the electric power industry in Taiwan by an examination of the death certificates between 1971 and 1985. He observed an elevation of liver and brain tumors and leukemia with odds ratios in the range 1.3 to 2 [Lin 87].

Estimation of exposure is the main problem with these studies which use data based on occupational classification. The data are classified by job titles or general occupational codes. These "electrical occupations" in some cases include electrical and telecommunication engineers who are no more exposed to ELF fields than the average individual. Even electricians often work with circuits turned off so that their exposure may not be significantly different than that of others. In addition, these jobs often involve exposures to other environmental agents such as chemicals, and this can confounds the findings.

\subsection{General Conclusions on the status of understanding of the ELF fields-cancer association}

The question of association between cancer and ELF electromagnetic fields first arose because of the work on childhood cancer in Denver. Studies since then have yielded mixed results. The most thorough epidemiological study by Savitz and the cellular level studies described in Section 3 provide some evidence to support the possibility that ELF field exposure can act as a cancer promoter. Overall the evidence now available is too weak to allow firm conclusions either way, 
Because epidemiological studies involve human populations, they do not have the problems associated with extrapolating from cell to whole animal or from animal to human. On the other hand, the epidemiological studies which have been completed todate are all retrospective and so may involve confounding effects and bias arising from such lack of control. Prospective epidemiological studies which can be expected in the future, will have fewer such difficulties, but even in this case, because the subjects are people with several other activities to pursue, problems of control will remain. No single epidemiological study can demonstrate causation. But a series of carefully designed studies which all indicate a positive association can provide persuasive evidence for causation especially if there are also supportive cellular and animal data. If $60 \mathrm{~Hz}$ fields do pose cancer risks, it will be some time before this stage of understanding is reached. A number of improved epidemiological studies will be needed and more importantly, a series of animal cancer promotion studies will be needed. Largely because of limited research budgets, no animal promotion studies have yet been completed. 


\section{General Conclusions About Biological Effects of ELF Fields and Their Implication}

As the preceding discussions have indicated, there is now a very large volume of scientific findings based on experiments at the cellular level and from studies with animals and people which clearly establish that low frequency magnetic fields can interact with, and produce changes in, biological systems. While most of this work is of very high quality, the results are complex. Current scientific understanding does not yet allow us to interpret the evidence in a single coherent framework. Even more frustrating, it does not yet allow us to draw definite conclusions about questions of possible risk or to offer clear science-based advice on strategies to minimize or avoid potential risks.

Of the effects discussed, the central nervous system effects including circadian effects in animals and the possibility of cancer promotion appear most worthy of concern with respect to public health effects. These are now summarized.

\subsection{Central Nervous System Effects}

As a system that uses low frequency fields for its intracellular communication and function, the brain and central nervous system are natural candidates for interaction with fields. The implications of tissue or cellular level in vitro experiments for the whole organism are not clear. But the animal studies, including the circadian variations introduced by fields, indicate that:

1. Field-CNS interactions may have dependencies which are at very specific frequencies and intensities, and may vary with the background static fields present, the time of day and the duration of exposure.

2. Developing nervous systems maybe particularly susceptible and effects maybe latent, manifested only in specific situations or later in time.

3. More than any other agent known, except perhaps some psychotropic drugs, ELF fields are specific: with respect to the regions of the brain tissue affected and the point of administration in the circadian rhythm.

How and whether these findings have public health implications remains unclear. What is clear is that these findings about subtle and complex effects demand a carefully planned research agenda in this area.

\subsection{Cancer Promotion}

The following points summarize the key experimental results that are consistent with a possible association between exposure to ELF fields and the occurrence of cancer:

1. ELF fields are not known to cause any chromosomal damage, and, hence, are not likely initiators of cancer.

2. Some cellular level experiments indicate that the cell membrane is the site of the interaction between ELF fields and the cell. The membrane site responsible for this action has also been shown to be a receptor for chemical cancer-promoters.

3. ELF fields have been shown to increase ornithine decarboxylase (ODC) activity. All known cancer promoters stimulate ODC. However, the converse is not true. Many agents that promote ODC activity are not cancer promoters.

4. Alterations in protein synthesis, in immunological and hormone status, and in metabolic competence via circadian shifts can all contribute to the progress of initiated cancer. To the extent that ELF fields play a role in those, they might have an effect on tumor growth or 
indeed tumor inhibition. The increase in ODC activity noted above is indicative of growth enhancement rather than inhibition.

5. Pineal melatonin depression has been associated with cancer growth, and administration of melatonin has been found to slow the growth of cancer. ELF fields depress pineal melatonin levels in animals.

6. Functions of gap junctions are disrupted by ELF fields. Similar disruptions are produced by other known chemical promoters.

7. Epidemiologic studies of ELF exposures and cancer show a weak association between ELF field exposure and nervous system cancer and leukemia.

While the above arguments are consistent with the hypothesis that ELF fields may play a role in cancer or tumor development, none of these constitutes proof or even necessarily a strong indication that it does. 


\section{Major Programs and Funding Levels for ELF Bioeffects Research}

In the long run, better scientific understanding is the only way to resolve problems posed by powerfrequency fields. Yet funding for field-effects research has been irregular over the years and current levels of federal support are modest. A history of the research funding provided by the six largest programs is shown in Figure 8-1. These and other research programs are described below.

The U.S. Navy played an important early role in research on the bioeffects of ELF electric and magnetic fields. In 1968, the Navy proposed to build an ELF submarine communications facility in northern Wisconsin that would have covered many thousands of square miles. In response to concerns raised by the people of Wisconsin and to comply with the recently enacted National Environmental Policy Act, the Navy launched a large laboratory research program that examined the effects of ELF field exposures on many animal and plant species [Rozzell 74]. This program funded about $\$ 8$ million worth of research between 1969 and 1977 [Abromavage ??]. The Navy now has two operating ELF transmitting facilities, one in Wisconsin and one in Michigan. The Navy has continued to sponsor ecological field studies in the vicinity of these transmitters since they began operation. Funding for these program is currently about $\$ 2$ million per year [Abromavage ??].

Over the last decade, the Department of Energy's (DoE) Office of Energy Storage and Distribution has been the chief federal source of support for research on the possible biological effects of lowfrequency fields. DoE's fiscal 1988 budget of $\$ 2,2$ million is detailed in Table 8-1. Congress has allocated $\$ 3.0$ million for DoE in fiscal 1989 [MWN 88a].

Several laboratories of the Environmental Protection Agency (EPA) have had smaller research programs involved in both exposure- and effects-related studies. Because of federal budgetary pressures, most of EPA's projects dealing with ELF fields were shut down in 1986 [MWN 86].

The federal government has not been the only source of research support in the United States. From 1982 through 1986, the state of New York operated a $\$ 5$ million research program on field effects administered by the New York Department of Public Health, with money largely provided by the state's electric utilities. Although this program has now ended, the research it generated has opened interesting new issues and produced several very useful results. Another useful but smaller state-funded venture has been the Maryland Power Plant Siting Program, which has supported database development and dosimetric studies at the Johns Hopkins Applied Physics Laboratory. California recently adopted a bill requiring the state's larger utilities to fund a two-year, $\$ 2$ million research project on the biological effects of ELF electromagnetic fields [MWN 88b].

The electric utility industry has also been involved in supporting research on ELF field effects. Utility support began as early as 1962, when American Electric Power Company (AEP) funded two smallscale studies at Johns Hopkins University, one of EHV lineworkers and one of mice exposed to strong electric fields. Several years earlier, AEP had become the first U.S. utility to build an EHV transmission line. The Bonneville Power Administration is another utility that has made a significant contribution to research. BPA has provided about $\$ 200$ thousand per year (in ' 87 dollars) for the past decade, primarily for environmental and livestock studies [Lee 87], Several other utilities, most notably Southern California Edison, have mounted fields research programs. Together, these sources have provided about $\$ 3$ million in funding over the last decade [Sahl 87].

The Electric Power Research Institute (EPRI) has supported a substantial amount of work since the 
Funding for ELF Bioeffects Studies

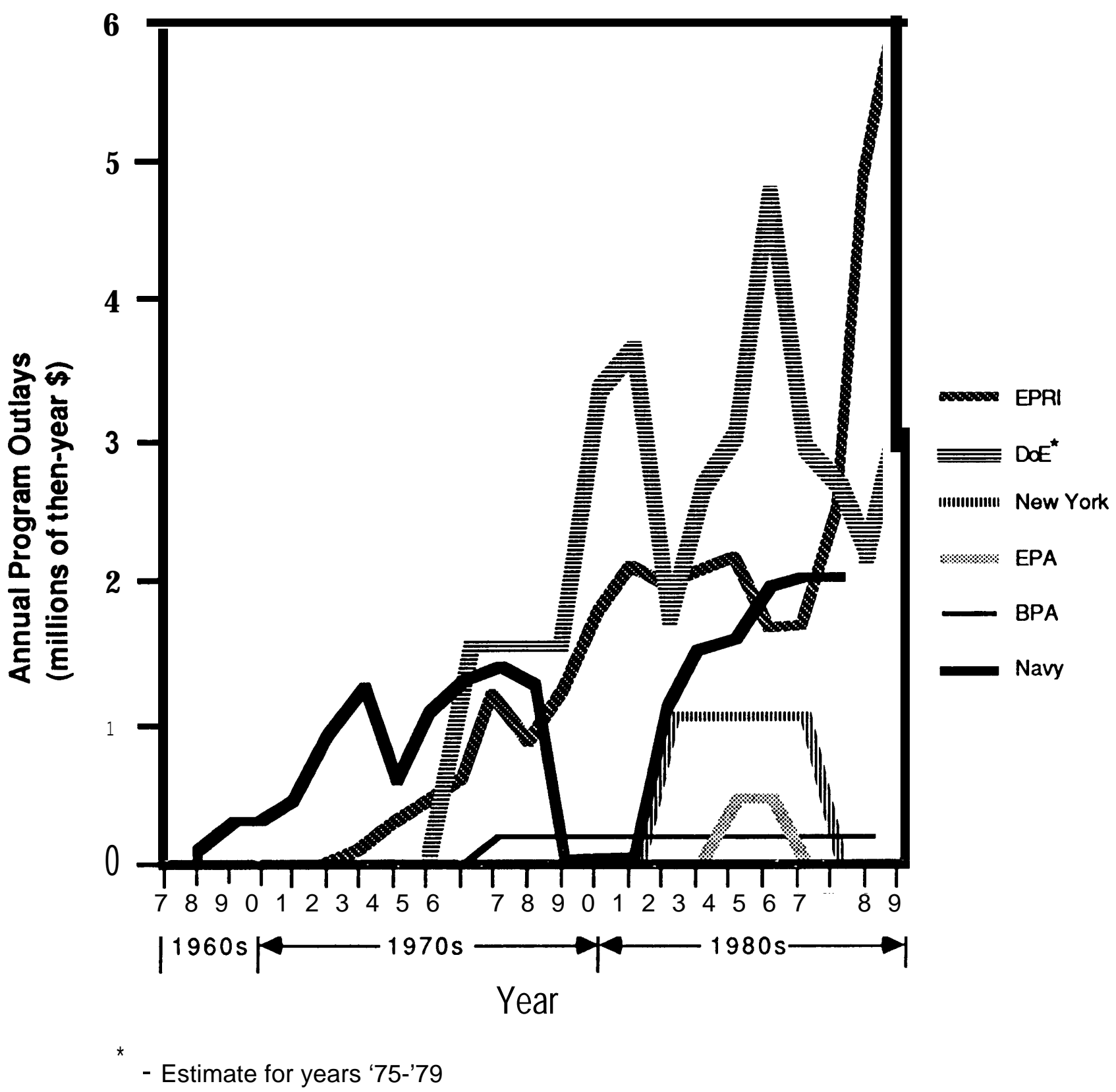

Figure 8-1: History of funding for ELF bioeffects studies in the U.S. from 1968 to the present. From [Abromavage ??, BEMS 86, Gyuk 88, MWN 88a, Sussman 88]. 
Table 8-1: U.S. Department of Energy 1988 Funding for ELF Electric and Magnetic Fields Research. From [Gyuk 88].

Project Site

Project Description

'88 Contract Amount

\begin{tabular}{|c|c|c|}
\hline Battelle Pacific Northwest Laboratory & Pineal gland melatonin & $\$ 600,000$ \\
\hline Argonne National Laboratory & Circadian rhythms, mice & $\$ 325,000$ \\
\hline MidWest Research Institute & $\begin{array}{l}\text { Response of human volunteers to } \\
60 \mathrm{~Hz} \text { electric and magnetic fields }\end{array}$ & $\$ 300,000$ \\
\hline Pettis VA Hospital, Loma Linda, CA & Cellular mechanisms & $\$ 225,000$ \\
\hline Southwest Research Institute & Baboon behavior & $\$ 90,000$ \\
\hline University of Rochester & $\begin{array}{l}\text { Rodent behavior under lithium } \\
\text { cyclotron resonance }\end{array}$ & $\$ 170,000$ \\
\hline Lawrence Berkely Laboratory & $\begin{array}{l}\text { Reaction of leukaemia cells } \\
\text { to electromagnetic fields }\end{array}$ & $\$ 126,000$ \\
\hline National Bureau of Standards & $\begin{array}{l}\text { Effect of temperature on the emission } \\
\text { of ions from high voltage DC lines }\end{array}$ & $\$ 50,000$ \\
\hline Program management & & $\$ 300,000$ \\
\hline Total & & $\$ 2,186,000$ \\
\hline
\end{tabular}

early 1970s. EPRI support declined slightly in recent years but is now expanding, from $\$ 1.7$ million in FY 1986 to a projected \$6 million in FY 1990 [MWN 88c].

Many other nations have active fields-research programs. In approximate order of current funding commitments, these include Sweden, the U. K., West Germany, Canada, Japan, Italy, France, Finland, and Norway.

Sweden's program is the largest, currently running at about 11 million krona ( $\$ 1.9$ million) per year. Swedish research includes projects in epidemiology, exposure assessment, and cancer induction and promotion. These projects are funded primarily by the Sweden's State Power Board and Sweden's National Institute of Occupational Health.

Research in the U.K. is funded primarily through the Central Electricity Generating Board (CEGB). 
Since roughly 1980, the CEGB has spent 3.5 million pounds (about $\$ 7$ million ) on fields-related research including epidemiological, laboratory, and human exposure studies. Recently the CEGB announced a doubling of its annual commitment to 1 million pounds ( $\$ 1.8$ million) per year. The CEGB's parent body, the Electricity Council, has also contributed to fields-related research in the U. K., but at a much lower level than the CEGB.

Using support from both public and private sources, the West Germans are funding a half-dozen projects including animal teratology experiments, in vitro studies, and measurements of human exposure.

Canada's Ontario Hydro and Hydro Quebec have been actively involved in exposure related research for some time and have recently begun an animal cancer study. They also have active programs in high voltage DC (direct current) field and ion effects.

Japanese utilities have funded a number of studies of electric field dosimetry over the last few years. They have also supported a study at Southwest Research Institute on the effects of electric fields on baboon behavior. rodents.

Italy's programs are entirely utility funded and include electric field studies with chickens and 


\section{Regulatory Activity and Exposure Standards}

There are growing pressures for states to take regulatory action to protect citizens against possible risks posed by power frequency fields. Major transmission line projects in New York, Montana, Florida and several other states have encountered considerable opposition. The courts have now become involved [Alvarez 86, Kelly 86]. In 1985, a Texas a County Civil Court ordered Houston Lighting and power to pay $\$ 25$ million in punitive damages on the grounds that in building a $345 \mathrm{kV}$ transmission line within 60 meters of a school, and in full compliance with all laws of the State of Texas, the utility had acted "with callous disregard for the safety, health and well-being of... the children...". The Texas Court of Appeals has since denied the $\$ 25$ million award but affirmed the lower court's finding that there are potential health effects associated with exposure to powerline fields. Prevented from using the transmission line pending its appeal, Houston Lighting and Power rerouted the line around the school property at a cost of $\$ 8.6$ million [MWN 87]. In New York, a group of land owners has filed a $\$ 66.5$ million class-action suit claiming that the fear of health effects has had a negative impact on the value of properties along a new $345 \mathrm{kV}$ transmission line. It is estimated that over $\$ 1.5$ million in attorney and witness fees had been spent on the case through August 1988 [MWN 88d]. Other less dramatic incidents are occurring all over the country.

To date, most of those pressures are directed toward the control of transmission lines, but it seems likely that similar pressures will grow for distribution lines, at least for those which are visible because they are above ground. While fields from house wiring and appliances can involve field exposures that are comparable to those associated with transmission and distribution lines (Figures 2-5 and 2-8), pressures to control fields from house wiring and appliances are likely to be slower in building. [Morgan 85].

If pressed to regulate field exposures, the natural instinct of most state regulatory authorities, based on experience with other environmental agents, is to implicitly assume that more is worse and impose field strength limits. To date, seven states have taken regulatory actions to limit the intensity of the electric field on transmission line rights-of-way. Recently, the state of Florida adopted limits on both electric and magnetic fields from transmission lines. A brief summary of the existing field limits is shown in Table 9-1.

Table 9=1: State regulations that limit field strengths on transmission line rights of way (RoW).

\begin{tabular}{|c|c|}
\hline State & Field limit \\
\hline 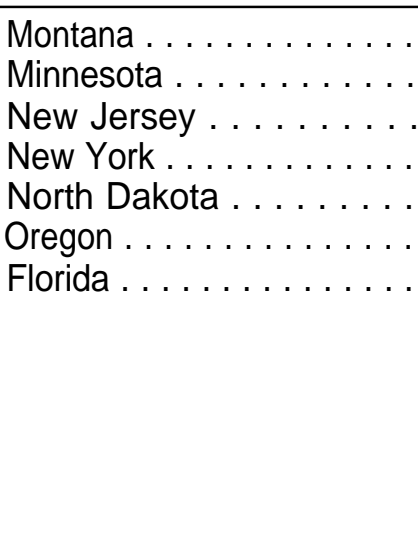 & $\begin{array}{l}1 \mathrm{kV} / \mathrm{m} \text { at edge of RoW in residential areas } \\
8 \mathrm{kV} / \mathrm{m} \text { maximum in RoW } \\
3 \mathrm{kV} / \mathrm{m} \text { at edge of RoW } \\
1.6 \mathrm{kV} / \mathrm{m} \text { at edge of RoW } \\
9 \mathrm{kV} / \mathrm{m} \text { maximum in RoW } \\
9 \mathrm{kV} / \mathrm{m} \text { maximum in RoW } \\
10 \mathrm{kV} / \mathrm{m} \text { maximum for } 500 \mathrm{kV} \text { lines in RoW } \\
2 \mathrm{kV} / \mathrm{m} \text { maximum for } 500 \mathrm{kV} \text { line at edge of RoW } \\
8 \mathrm{kV} / \mathrm{m} \text { maximum for } 230 \mathrm{kV} \text { smaller lines in RoW } \\
2 \mathrm{kV} / \mathrm{m} \text { maximum for } 230 \mathrm{kV} \text { and smaller lines at edge of RoW } \\
200 \mathrm{mG} \text { for } 500 \mathrm{kV} \text { lines at edge of RoW } \\
250 \mathrm{mG} \text { for double circuit } 500 \mathrm{kV} \text { lines at edge of RoW } \\
150 \mathrm{mG} \text { for } 230 \mathrm{kV} \text { and smaller lines at edge of RoW }\end{array}$ \\
\hline
\end{tabular}


Despite the proliferation of regulations based on field strength, the discussion in Section 2.5 makes it clear that regulatory approaches which set quantitative "safe" field strength limits (such as $1 \mathrm{kV} / \mathrm{m}$ at the edge of the right-of-way) cannot be supported on risk management grounds, given available scientific understanding. Such a standard offers no assurance that it will reduce the overall level of risk. 


\section{Policy Implications}

There is of course nothing new about a possible environmental health risk for which our scientific understanding is incomplete. Legislators and regulators have been dealing with such risks for decades. But, when we look with care at the scientific understanding that is available for $60 \mathrm{~Hz}$ and other low frequency electromagnetic field exposure, we discover that this particular problem maybe very different from previous problems in environmental risk in several ways.

First, the quality of the science that is now available is remarkably high. In contrast to a number of other known or suspected environmental health risks the uncertainty in our understanding about low frequency electromagnetic fields comes not from the quality of the science but from the great complexity of that science. It is now clear that $60 \mathrm{~Hz}$ and other low frequency electromagnetic fields can interact with individual cells and organs to produce biological changes. The nature of these interactions is subtle and complex. The implications of these interactions for public health remain unclear, but there are legitimate reasons for concern.

Second, because of the complexity of the science, the strategies which legislators and regulators have evolved to deal with other uncertain environmental health risks may not lead to effective results for this possible risk. Most conventional approaches to environmental regulation assume that "if an agent is bad more of it is worse". This assumption allows regulatory activity to begin before full scientific understanding is available. Because of experimental evidence of thresholds, windows, and similar phenomena (see the discussion in Section 3 and the summary in the preceding section), it is unclear whether the assumption that "more is worse" can appropriately be applied to $60 \mathrm{~Hz}$ fields. The implication of this is that practical regulatory standards which set a simple "safe" field strength limit can not be adequately suppported by the science that is now available. Such standards could prove expensive and might not have positive benefits. In some circumstances field strength based standards could arguably even do more harm than good. This conclusion raises the difficult policy question, if standard approaches may not work, what alternatives, do we have available? We explore this question at some length in the discussion below.

Third, the public discussion of $60 \mathrm{~Hz}$ fields has been almost exclusively limited to the context of high voltage transmission lines. Running across the country for great distances on large steel or wooden structures, high voltage transmission lines are visibly compelling objects. As such they offer powerful symbolic value and are a natural target for attention ${ }^{17}$. However, if there are serious public health consequences associated with $60 \mathrm{~Hz}$ fields the discussion in Section 2 in this report suggest that the fields associated with distribution lines (e.g. the wood poles in the street), building wiring, and appliances (e.g. electric blankets) could be the primary source of public health impact. To the extent this proves true, it will be important for legislators, regulators, and others to take care to think of this issue as a problem of field exposure rather than as a problem of high voltage transmission lines. Otherwise, enormous attention may be devoted to one, possibly minor, source of public exposure, while ignoring many other, possibly major, sources of public exposure.

Why not just use the standard techniques of probabilistic risk assessment and risk analysis to

\footnotetext{
${ }^{17}$ In the heydays of federal power projects (TVA andBonneville) and of rural electrification, high voltage transmission lines 'ere widely used as a political symbol to represent the advancement of democracy through economic development. For example they figured prominently in the promotional posters and other materials developed by these projects.
} 
decide how serious the possible risks of human exposure to $60 \mathrm{~Hz}$ fields maybe and develop appropriate regulatory recommendations from these studies? Under support from the $U$. S. Department of Energy we have tried to do this [Morgan 87a, Morgan 87b, Morgan 87c, Morgan 87d] but have found it impossible to get very far. The basic problem precluding risk analysis is the inability to define dose. We do not yet know what attribute, or combination of attributes, of the field produces public health effects. (For simplicity we will not continue to say "if any" after each mention of the word *'effects" in this discussion. However, readers are reminded that while biological effects have been clearly demonstrated, the existence of adverse public health consequences from $60 \mathrm{~Hz}$ field exposure is still an open question. Thus, whenever the word effects appears in this discussion, the phrase "if any" is implicitly assumed.) We also do not know the relationship between these field attributes and the level of effects. We do have a significant amount of experimental evidence that suggests that the relationship between field exposure and effects may be very non-linear, and may not increase systematically with field strength. In work we did several years ago [Morgan 83] we showed that it is possible to develop a series of risk assessment models each one of which assumes a different plausible relationship between exposure and effect and each one of which assumes a range of possible alternative values to define the strength and other characteristics of those relationships (parametric analysis). This approach can produce page after page of impressive looking quantitative results -- so many alternative possible outcomes that they are of absolutely no use from a policy perspective. Similarly, attempts to establish "upper bounds" on the estimates of possible health impact have produced bounds that are too lose to be of much use in policy considerations [Morgan 87e].

\subsection{Policy Alternatives}

The conventional approach to regulating environmental agents such as ionizing radiation or chemical pollutants is in terms of a standard based on a safe or acceptable level or concentration. When the details of the science are not known, risk managers can still move in the right general direction by assuming "if some is bad, more is worse" and regulating to reduce the levels of exposure. As we outlined in Section 9, without thinking critically about the underlying differences in the science, this same thinking has been applied by a number of state regulatory agencies to the topic of $60 \mathrm{~Hz}$ fields, with the result that several states have now promulgated fields strength standards for transmission lines which are at least talked about as safety standards.

There are however a variety of powerful forces that work to promote the adoption of simple field strength safety standards. This is the approach that regulatory agencies have habitually adopted. There are a variety of legal and institutional precedents which make a field strength standard the natural way to proceed. Because field related controversies have made it increasingly difficult for utilities to construct new transmission facilities, many utilities have recently begun to favor the issuance of such standards, so that they can say "we meet the standards" and get on with the job. But, while simple field strength standards may be administratively convenient for both regulators and utilities, they unfortunately can not be justified on the basis of the available science. If they are represented as assuring safety they may produce a false sense of protection, and in some circumstances could arguably do more harm than good.

Better scientific understanding may in the future clearly demonstrate the existence of adverse public health effects from field exposure and may point the way to specific risk management regulations. But, for the moment we have to operate with what we have. Available policy options include the following: 1. Do nothing until the science becomes better. 
2. Make public information available but take no additional actions.

3. Adopt a field strength safety standard approach to transmission line fields based on the fiction that the numbers are supported by a review of the science. Ignore fields from all other sources.

4. Adopt a "similarity" based approach to transmission line fields which makes the exposures that people receive to these fields "similar" to those they receive from other sources in modern life. Ignore fields from all other sources.

5. Adopt a "prudent avoidance" strategy. That is, look systematically for strategies which can keep people out of $60 \mathrm{~Hz}$ fields arising from all sources but only adopt those which look to be "prudent" investments given their cost and our current level of scientific understanding about possible risks.

In parallel with these options are a set of research options which we discuss in the final paragraphs of this section.

Up until a few years ago we believe that option 1, "do nothing until the science becomes better", was preferred by the majority of informed people dealing with this topic. There are still people who argue vigorously for this option, but their numbers are declining both because of increasingly suggestive scientific findings and because of growing levels of public concern. At least in the short run, Some state regulatory agencies do appear to be successfully adopting a variant of this strategy, either because they have not had a recent request for new transmission line construction that has raised the issue or because they have been able to mount a series of ongoing studies of the problem.

Available public information on this topic is not as good as it should be. Press accounts tend to be simplistic and inflammatory. There are a few good brochures available through utilities, but there is reason to suspect that the public does not place great confidence in information on this topic provided by utility sources [Lee 86, IUS86 86]. There is a clear need for good balanced semi-technical and nontechnical treatments of this topic from "neutral" government and private sources. Until recently many people familiar with the issues would have argued that active programs of public information might do more harm than good by raising concerns in large numbers of people before any answers were available. While a few might still make this argument, most would now agree that the issue has moved squarely on to the public agenda and that there is now a clear need for accurate and balanced information to inform public discussions and debates. Public information programs can take two forms, active programs which push the dissemination of information, and passive programs which provide information on request. To date most programs have been of this second variety. Whether programs of information alone are now a sufficient response to the issue of $60 \mathrm{~Hz}$ fields is a question which generates vigorous debate among informed workers active on this issue. Our personal assessment is that today a majority would probably argue that information alone is not sufficient.

Policy option three is to:

"Adopt a field strength safety standard approach to transmission line fields based on the fiction that the
numbers are supported by a review of the science. Ignore fields from all other sources."

It appears that this option is now being pursued by a number of state regulatory agencies. As we noted above it is also supported by a number of utilities as a simple strategy to control the issues and get on with the job. There are three ways in which a field strength based approach to transmission line field regulation can be pursued. The first is to assert that the standard grows directly from and is supported by a careful assessment of the field effects literature. While administratively convenient, our reading of the 
scientific literature says that the literature can not currently support such a standard. The second is not to base the standard on field effects of the sort discussed in this paper but rather to base it on considerations of safety arising from factors such spark discharge caused by induction effects in conducting objects near transmission lines. If such a strategy is pursued there is likely to be a temptation to "fudge" a bit by leaving the public communication unclear so as to imply that other health effect considerations, of the kinds discussed in this paper, have been included. Finally there is the practical consideration that at least for electric fields transmission line fields much above about $10 \mathrm{kV} / \mathrm{m}$ are clearly precluded by safety considerations and fields much below about $1 \mathrm{kV} / \mathrm{m}$ are at the level widely encountered elsewhere in the environment. This leaves a range of only a factor of ten. The temptation is to arbitrarily pick some number in this range in order to have a standard that will simplify life. Our judgment is that such considerations may have underlain some of the current state field strength standards.

As we discuss below, a field strength standard may also have a role to play in a "prudence* ${ }^{* \star}$ based approach to field exposure control. The difference however is two fold. Conventional standards maintain the position that they represent a "safe" level below which there is little or no risk. And, a conventional transmission line field strength standard deals only with exposure from transmission line sources, ignoring all others, or perhaps assuming that all others constitute "acceptable" exposures.

This leads us to our fourth option:

Adopt a "similarity" based approach to transmission line fields which makes the exposures that people receive to these fields "similar" to those they receive from other sources in modern life. Ignore fields from all other sources.

Similarity based control sets out to make people's exposures to transmission line fields as "similar" as possible to the exposures we receive from all the other fields in our day-to-day lives: exposure from the fields from the power lines out in the street, the wiring in the buildings we live and work in, and the appliance we use during the day and sleep with at night. The definition of "similar" can get technically complicated I[Florig 86, Morgan $87 \mathrm{c}$ ], but the idea is simple enough. A similarity based approach to transmission line field control can be justified on two possible grounds:

1. "Acceptability" - In this case the argument is that the fields to which we are all exposed from other sources constitute a socially acceptable level of risk. By making transmission line field exposures similar, we make them socially acceptable.

2. "Equity" - In this case the argument is that if transmission line fields are made similar to the fields to which we are all exposed from other sources then we are not asking residents who live along transmission line right-of-ways to bear field related risks that are any different from those born by all members of modern society.

We suspect that similarity considerations have entered, at least informally, into the decision making of several state regulatory agencies. In the case of New York State, the standard was explicitly chosen to make the fields of higher voltage lines similar to those of the $345 \mathrm{kV}$ lines already in wide use in the state. However, recent legal actions cast doubt on the state's assumption that the $345 \mathrm{kV}$ lines are "widely accepted".

Our fifth and final option is a strategy of "prudent avoidance" of field exposures. By avoidance we mean taking steps to keep people out of fields, both by re-routing facilities and by redesigning electrical systems and appliances. By prudence we mean undertaking only those avoidance activities which carry 
modest costs ${ }^{18}$. When, as individuals, we think a risk may exist but we are not sure, we exercise prudence. For example, broccoli and cauliflower may contain anti-carcinogens. Dietary fiber may help to reduce the risk of certain cancers. Conversely char-grilled meats may carry increased risks of cancer. The evidence on these things is suggestive but inconclusive. As a matter of prudence many people have tried to increase the frequency with which they eat cauliform vegetables, increase their fiber intake, and reduce the amount of char-grilled meat they eat. But reasonable people do not rent a helicopter to fly high fiber bread in to them when they spend a week at a mountain ski resort which serves only regular bread. Families who eat meat, would not buy lobster for their kids every night for a week at that same ski resort if it is the only meat on the menu that is not charbroiled. Nor do reasonable people rent their own refrigerated truck to supply them with broccoli and cauliflower when they travel in places where these foods are not available. Such steps go beyond prudence. At the least they would be foolishly expensive, at the worst, signs of serious paranoia.

What would constitute prudence in the context of keeping people out of $60 \mathrm{~Hz}$ fields? Here are a few possibilities:

. attempt to route new transmission lines so that they avoid people;

. widen transmission line rights-of-way;

. develop designs for distribution systems, including new grounding procedures, which minimize the associated fields;

. develop new approaches to house wiring that minimize associated fields;

. redesign appliances to minimize or eliminate fields.

If we decide to do these things we have to ask how do we avoid going overboard... how do we avoid the equivalent of renting the helicopter? The answer lies in asking how much we should be prepared to invest in avoiding exposing people to fields. It is fairly easy to set an upper bound on the amount we should be willing to spend. Clearly it makes no sense to invest more per person-exposure avoided than we invest per death avoided for various known risks in our society. In other writings we have used the rates at which our society invests in avoiding known risks of death and injury to develop an upper bound on the rates at which it would be prudent to invest in field avoidance [Morgan 87b, Morgan 87c]. We conclude that it might be possible to justify investment rates of up to some thousands of dollars of personexposure avoided, but not possible to justify rates of investment in field avoidance activities that are significantly higher than this. Thus, for example, while it might make sense to work to avoid exposing people in siting new lines, in most cases, with our current knowledge, it would not make sense to tear out and rebuild old lines. Similarly it might make sense to redesign new appliances to reduce fields exposure if this can be done for small increments in their cost. It might even makes sense to selectively replace a few old appliances, such as electric blankets, with new "field-free" versions. But it probably would not make sense to throw out all old appliances before they wear out and replace them all immediately with new "field-free" ones.

To date there has been very little research on the design of strategies to reduce field exposures. We have done a small amount of work ourselves, principally in student projects [CarnegieMellon 84]. This work suggests that in many cases low-field or "no-field" solutions maybe possible at economically

\footnotetext{
${ }^{18}$ Sections of this discussion draw directly from a paper by the authors which recently appeared in Public Utilities Fortnightly [Morgan 88]
} 
reasonable levels. For example a low-field electric blanket might be designed by using concentric conductors in the heating elements, by using shielded twisted pair heating elements, or by using heated fluid.

In the case of transmission lines we have developed proposals for specific strategies based on transmission line siting fees which could be used to implement prudent avoidance. The basic idea is to charge the utility a fee of $\$ X$ for each person who lives within a given distance ${ }^{19}$ of the line. If properly implemented this approach should provide incentives for the utility to avoid exposing people up to a marginal investment rate of $\$ X$ per person-exposure avoided. We have argued that the revenue generated by such a scheme should be used to support $60 \mathrm{~Hz}$ health effects research. Details on this strategy can be found elsewhere [Morgan 87d, Morgan 88]. If a fee based approach is considered unacceptable, various second-best administrative procedures may be used to achieve similar goals. For example, if it was undertaken with the explicit recognition that it did not represent a "safe" exposure level, a field strength standard might be justified in terms of *'prudent avoidance" arguments.

Society has trouble doing collectively things that we do as individuals all the time. In particular, the notion of exercising social prudence on a possible but uncertain risk such as $60 \mathrm{~Hz}$ fields is a nonstandard strategy for social risk management. To implement it will require some behavioral, political and perhaps even some legal changes. For example, as noted above, with some careful engineering, the field exposures associated with many appliances could probably be dramatically reduced or eliminated, at only modest increases in price [CarnegieMellon 84]. Suppose, that in the spirit of prudent field avoidance, an electric blanket company decides to redesign their blankets to make them "field free". It seems only a matter of time before the company will be facing litigation from leukemia victims which argues that the electric blankets caused their cancer and that the fact that the company has recently redesigned their blankets "proves" their claim, since otherwise why would the company have gone to the trouble to introduce the new design.

To date there has been very little work done on field avoidance strategies. There is clearly a need for a series of careful engineering, economic and legal studies. While DoE supports research on $60 \mathrm{~Hz}$ health effects, primarily from a transmission line perspective, neither they nor any other agency appears to have yet assumed responsibilities that span the full problem, including distribution, building wiring and appliances. Clearly, such research is needed if we wish to be able to develop a set of coherent and rational responses to the problem of general social exposure to $60 \mathrm{~Hz}$ fields.

\subsection{Strategies for Research}

Recent years have seen dramatic developments in the science which have prompted many observers to conclude that the issue of possible $60 \mathrm{~Hz}$ health risks should be taken seriously. In parallel with these developments, the past several years have also witnessed a marked decrease in the level of federal research support in this area which has only recently begun to reverse. The reductions do not appear to have sprung from any deliberate choice to reduce $60 \mathrm{~Hz}$ field effects research. Rather, they appear to have been the byproduct of high level efforts to limit the level of overall federal expenditures.

\footnotetext{
${ }^{19}$ The avoidance distance used in such a scheme would typically be significantly greater that the widths Of current transmission line rights-of-way, which would be retaineand could to widened.
} 


\section{References}

[Abromavage 87.'] Abromavage, M. M., Engineering Advisor, IIT Research Institute, Washington, D.C. Personal communication, October, 1987.

[Adey 81] Adey W.R. Tissue Interactions with Nonionizing Electromagnetic Fields. Physiological Reviews $61: 435-514,1981$.

[Adey 82] Adey, W. R., S.M. Bawin, and F. Lawrence. Effects of Weak Amplitude-Modulated Microwave Fields on Calcium Efflux From Awake Cat Cerebral Cortex. Bioelectromagnetics 3:295-307, 1982 .

[Adey 84] Adey W.R. and A.F. Lawrence (editors). Nonlinear Electrodynamics in Biological Systems. Plenum Press, New York, 1984.

[Adey 86] Adey W.R. Electromagnetic Fields, Cell Membrane Amplification and Cancer Promotion. Technical Report, Presented at the National Council on Radiation Protection and Measurements Annual Meeting, National Academy of Sciences, Washington, D. C., 1986.

[Adey 87] Adey W.R. Testimony presented to the U.S. House of Representatives, Subcommittee on Water and Power Resources, October 6. 1987.

[AIBS 85] Committee on Biological and Human Health Effects of Extremely Low Frequency Electromagnetic Fields. Biological and Human Health Effects of Extreme/y Low Frequency Electromagnetic Fields: Post- 1977 Literature Review. Technical Report AD/AI 52731, Prepared for the Naval Electronic Systems Command by the American Institute of Biological Sciences, 1985.

[Albert 84] Albert E., G, Cohen, L. Avellino, G. Kornhouser and A. Yoshioka. Electron Microscopic Observations on Rat Cerebellum and Hippcampus After Exposure to 60-Hz Fields. Technical Report, Abstract, Sixth Annual Meeting of the Bioelectromagnetics Society, Atlanta, GA, 1984.

[Alvarez 86] Alvarez, C. The EMF Issue: The Florida Regulatory and Legal Experience. Technical Report, Proceedings of the International Utility Symposium, September 16-19, Toronto, Canada, 1986. Available from Ontario Hydro, Ontario, Canada.

[ANSI 77] American National Standards Institute. National Electrical Safety Code. 1977.

[Axelrod 84] Axelrod J. and T.D. Reisine. Stress Hormones and Their Regulation. Science 224:452-459, May, 1984.

[Barnes 67] Barnes, H. C., A.J. McElroy and J.H. Charkow. Rational Analysis of Electric Fields in Live Line Working. IEEE Transactions on Power Apparatus and Systems PAS-86(4):482-492, April, 1967.

[Barnes 87] Barnes, F., H. Wachtel, D. Savitz, and J. Fuller. The Use of Wiring Configuration and Wiring Codes for Estimating Externally-Generated Electric and Magnetic Fields. 1987. Manuscript available from Frank Barnes, Dept. of Electrical and Computer Engineering, University of Colorado, Boulder, CO 80309.

[Barregard 85] Barregard L., B. Jarrnolm and E. Lungenthum. Cancer Among Workers Exposed to Strong Static Magnetic Fields. Lancet 2:892, 1985.

[Bassett 74] Bassett C. A. L., R.J. Pawluk and A.A. Pilla. Augmentation of Bone Repair by Inductively Coupled Electromagnetic Fields. Science 184:575-577, 1974.

[Bassett 82] Bassett C. A. L., N. Mitchelsl and S.R. Gaston. Pulsing Electromagnetic Fields in Unlimited Fractures and Failed Arthrodeses. Journal of the American Medical Association 247:623-627, 1982.

[Bawin 75] Bawin, S. M., L. Kaczmarek, and W.R. Adey. Effects of Modulated VLF Fields on the Central Nervous System. Annals of the New York Academy of Science 247:74-81, 1975. 
While research completed to date is sufficient to raise serious questions it is not sufficient to provide satisfactory answers or to point the way to action. Electric power plays a critically important role in the health of our nation. Already concerns have prompted vigorous public intervention and litigation which has significantly impeded the ability of private and public utilities to construct new power transmission facilities. Such protests will probably grow and it seems likely that similar concerns about fields will soon be raised at other levels. Without adequate science on which to base answers, the resulting contention could go on for many years and have costs significantly greater than the costs of the needed research.

While legislators, regulators and risk managers all have a role in determining the /eve/of research that is undertaken, they should obviously not become involved in specific decisions about the details of the research to be conducted. But, beyond the issue of level of support, there are several issues of research management that should concern these groups:

1. Because of its highly interdisciplinary nature (involving biology, biochemistry, physics and electrical engineering) a high level of quality control is required for research on the health effects of low frequency electromagnetic fields. Failure to exercise such control can lead to serious confusion. The current private and public research support programs have developed adequate quality control. If other actors, such as additional individual states, begin to support research, they need to take care to exercise similar control.

2. The overall program of research should include a balanced mixture of a) cellular level and other biological studies of mechanisms; b) whole animal studies; and c) epidemiological studies. No single type of study is likely to lead to the kind of complete understanding which will be necessary to make informed judgments about risk assessment and management, For example, while epidemiological studies maybe able to demonstrate health impacts in humans, cellular and animal studies are likely to be necessary to identify the dose-response mechanisms needed to develop effective strategies for risk management.

3. Care must be taken not to define the scope of the program too narrowly. The fundamental science of this problem appears to be complex and may still lead in unexpected directions. It is important to define the scope of the research widely enough so that important but unexpected developments will not be missed. An example will clarify this point. In the early years of work on this topic it was common for some observers to complain that any study done at a frequency other than $60 \mathrm{~Hz}$ was irrelevant. Had this position been enforced on the research then occurring, the important discovery of frequency windowing (Section 3) would have been missed and the correct interpretation of a number results would be impossible.

4. Management of the research program must be flexible and adaptive so as to be able to redirect attention as understanding evolves. For example, in the past the federal research program was too slow in phasing down support for rodent behavioral studies. Recently it has been too slow in mounting a series of laboratory animal cancer promotion studies. In order to avoid such problems regular strategic program reviews based on independent critical advice from health scientists, engineers and risk analysts, are necessary.

5. There is a risk of becoming too fixed on cancer as a single health effect of concern, The breadth of cellular and animal findings suggest that other public health effects, including psychological effects such as chronic depression, deserve some attention.

6. Insufficient attention has been directed at field exposures that result from sources other than high voltage transmission lines. A systematic characterization of the entire low frequency field environment to which people are exposed in normal modern life, is needed.

7. There has been almost no attention given to techniques for eliminating $60 \mathrm{~Hz}$ field exposures in modern life. A series of engineering studies, designed to explore the technical and economic feasibility of exposure avoidance, are needed. 
[Bawin 76] Bawin, S.M. and W.R. Adey. Sensitivity of Calcium Binding in Cerebral Tissue to Weak Environmental Electric Fields Oscillating at Low Frequency. Proceedings of the National Academy of Sciences 73(6):1999-2003, June, 1976.

[Bellossi 86] Bellossi A. Lack of an Effect of Static Magnetic Field on Calcium Efflux from Isolated Chick Brains. Bioelectromagnetics 7:381-386, 1986.

[BEMS 86] Bioelectromagnetics Society. Bioelectromagnetics Funding Survey.

Bioelectromagnetics Society Newsletter (68), May/June, 1986.

[Benz 87] Benz R. D., A.L. Carsten, J.W. Baum, and A.V. Kuchner. Mutagenicity and Toxicity of 60-Hz Magnetic and Electric Fields. Technical Report, Final Report to the New York State Power Lines Project, Wadsworth Labs, E-297, Empire State Plaza, Albany, New York, 1987.

[Blackman 82] Blackman, C. F., S.G. Benane, L.S. Kinney, W.T. Joines, and D.E. House. Effects of ELF Fields on Calcium-Ion Efflux from Brain Tissue In Vitro. Radiation Research 92:510-520, 1982.

[Blackman 85a] Blackman, C, F., S.G. Benane, D.E. House, W.T. Joines. Effects of ELF (1-120 Hz) and Modulated $(50 \mathrm{~Hz})$ RF Fields on the Efflux of Calcium lons from Brain Tissue In Vitro.

Bioelectromagnetics 6(1):1-11, 1985.

[Blackman 85b] Blackman, C. F., S.G. Benane, J.R. Rabinowitz, D.E. House, W.T. Joines. A Role for the Magnetic Field in the Radiation-induced Efflux of Calcium lons from Brain Tissue In Vitro.

Bioelectromagnetics 6(4):327-337, 1985.

[Blackman 87] Blackman, C. F., S.G.Benane, D.E. House, J.R. Rabinowitz, and W.T. Joines. ELF Electromagnetic Fields Cause Enhanced Efflux of Neutral Sugars from Brain Tissue In Vitro. Technical Report, Abstract, Ninth Annual Meeting of the Bioelectromagnetics Society, Portland, OR, June 21-25, 1987.

[Blackman 88] Blackman C. F., S.G. Benane, and D.E. House. Effect of Ambient Levels of PowerLine-Frequency Electric Fields on a Developing Vertebrate. Bioelectromagnetics 9(2), 1988.

[Blackwell 85] Blackwell R. P., and A.L. Reed. Effects of Electric Field Exposure on Some Indices of CNS Arousal in the Mouse. Bioelectromagnetics 6:105-107, 1985.

[Blackwell 86] BlackWell R. P. Effects of Extremely-Low Frequency Electric Fields on Neuronal Activity in Rat Brain. Bioelectromagnetics 7:425-434, 1986.

[Bowman 88] Bowman, J. D., D.H. Garabrant, E. Sobel, and J.M. Peters. Exposures to Extremely Low Frequency (ELF) Electromagnetic Fields in Occupations with Elevated Leukemia Rates. Applied Industrial Hygiene 3(6):1 89-194, 1988.

[Brown 70] Brown F. A., J.W. Hastings and J.D. Palmer. The Biological Clock: Two Views. Academic Press, New York, 1970.

[Burack 84] Burack, G. D., Y. J. Seto, S.T. Hsieh and J.L.Dunlap. The Effects of Prenatal Exposure to a $60-\mathrm{Hz}$ High Intensity Electric Field on Postnatal Development and Sexual Differentiation. Journal of Bioelectricity 3:451-467, 1984.

[Byus 86] Byus, C. V., S.E. Pieper and W.R. Adey. The Effects of Low-Energy 60-Hz Environmental Electromagnetic Field Upon the Growth-Related Enzyme Ornithine Decarboxylase. Carcinogenesis 8:, 1986.

[Cain 86] Cain C. D., M.C. Malto, R.A. Jones and W.R. Adey. Effects of 60-Hz Fields on Ornithine Decarboxylase Activity in Bone Cells and Fibroblasts. Technical Report, Contractors' Review Meeting, U.S. Dept. of Energy Office of Energy Storage and Distribution and the Electric Power Research Institute Health Studies Program, New York State Department of Health, Denver, CO, November, 1986.

[Cane 83] Cane E.E. and D.A. Savitz. Leukemia in Occupational Groups with Presumed Exposure to Electric and Magnetic Fields. New England Journal of Medicine 313:1476-1477, 1983. 
[Caola 83] Caola, R.J., Jr., D.W. Deno and V.S.W. Dymek. Measurements of Electric and Magnetic Fields in and Around Homes Near a 500 kV Transmission Line. IEEE Transactions on Power Apparatus and Systems PAS-1 02, 1983.

[CarnegieMellon 84]

Carnegie Mellon University Department Engineering and Public Policy, Department of Social Science, and the School of Urban and Public Affairs. Strategies to Reduce

Population Exposures to $60-\mathrm{Hz}$ Electromagnetic Fields, Final Report of the Fall Project Course, December. 1984.

[Carstensen 87] Carstensen, E.L. Biological Effects of Transmission Line Fields. Elsevier Publishing Company, New York, 1987.

[Chartier 85] Chartier, V. L., T.D. Bracken, and A.S. Capon. BPA Study of Occupational Exposure to 60-Hz Electric Fields. IEEE Transactions on Power Apparatus and Systems PAS-I 04(3):733-744, March, 1985.

[Chiabrera 84] Chiabrera A., M. Gratiarola, and R. Viviani. Interaction Between Electromagnetic Fields and Cells: Microelectrophoretic Effect on Ligands and Surface Receptors. Bioelectromagnetics 5:173-191, 1984.

[Cohen 86] Cohen M.M.In Vitro Genetic Effect of Electromagnetic Fields. Technical Report, Prepared for the New York State Power Lines Project, Wadsworth Labs, E-297, Empire State Plaza, Albany, New York, 1986.

[Cohen 87] Cohen M.M. The Effects of Low-Level Electromagnetic Fields on Cloning of Two Human Cancer Cell Lines (Co/o 205 and Co/o 320). Technical Report, Prepared for the New York State Power Lines Project, Wadsworth Labs, E-297, Empire State Plaza, Albany, New York, 1987.

[Cole $871 \quad$ Cole P. An Epidemiologic Perspective on Electromagnetic Fields and Cancer. Prepared for the Florida Department of Environmental Regulation, March. 1987.

[Deadman 88] Deadman, J. E., M. Camus, B.G. Armstrong, D. Cyr, G. Theriault, P. Heroux, and M. Plante. Occupational and Residential $60-\mathrm{Hz}$ Electromagnetic Fields and High-Frequency Electric Transients: Exposure Assessment Using a New Dosimeter. Journal of the American Industrial Hygiene Association 49(8):409-419, 1988.

[Delgado 82] Delgado J. N. R., J. Lealm, J.L. Monteagudo, and G.M. Garcia. Embryological Changes Induced by Weak, Extremely Low Frequency Electromagnetic Fields. Journal of Anatomy 134:533-551, 1982.

[Deno 78] Deno, D.W. and L.E. Zaffanella. Electrostatic and Electromagnetic Effects of Ultrahigh-Voltage Transmission Lines. Technical Report EL-802, Prepared for the Electric Power Research Institute, June, 1978.

[Deno 82] Deno, D. W., L.E. Zaffannella. Field Effects of Overhead Transmission Lines and Stations. Transmission Line Reference Book: 345 kVand Above. Electric Power Research Institute, Palo Alto , Ca, 1982.

[Deno 87a] Deno, D.W. and J.M. Silva. Transmission Line Electric Field Shielding by Objects. IEEE Transactions on Power Delivery PWRD-2(1), January, 1987.

[Deno 87b] Deno, D.W. Sources and Structure of Magnetic and Electric Fields in the Home. In L.E. Anderson, B.J.Kelman, R.J. Weigel (editors), Interactions of Biological Systems with Static and ELF Electric and Magnetic Fields. Proceedings of 23rd Annual Hanford Life Sciences Symposium, October 2-4, 1984, Richland, Washington, 1987. Available from NTIS. Order CONF-841041.

[Dowse 69] Dowse H.B. and J.D. Palmer. Entrainment of Circadian Activity Rhythms in Mice by Electrostatic Fields. Nature 222:564-566, 1969. 
[Dubrow 80] Dubrow R and D.H. Wegman. Occupational Characteristics of Cancer Victims in 'Massachusetts 1971-1973. Technical Report, National Institute for Occupational Safety and Health, Cincinnati, Ohio, 1980.

[Duffy 82] Duffy P.M. and C.F. Ehret. Effects of Intermittent 60-Hz Electric Field Exposure: Circadian Phase Shifts, Splitting, Torpor and Arousal Responses in Mice. Technical Report, Abstract, 4th Annual Meeting of the Bioelectromagnetics Society, Los Angeles, CA, June 28-July 2, 1982.

IEdmonds 81] Edmonds L. D., P.M. Layde, L.M. James, J.W. Flynt, J.D. Erickson and G.P. Oakley Jr. Congenital Malformation Surveillance: Two American Systems. International Journal of Epidemiology 10:247-252, 1981.

[Ehret 80] Ehret C. F., R.S. Rosenberg, G.A. Sacher, P.H. Duffy, K. R.Groh and J.J. Russell. Biomedical Effects Asscoiated with Energy Transmission Systems : Effects of 60-Hz Electric Fields on Circadian and Ultradian Physiological and Behavioral Functions in Small Rodents. Report to the Department of Energy Office of Energy Storage nad Distribution for the period Jan 1- Dec 1. 1980.

[Ellert 82] Ellert, F. J., S.A. Miske, C.J. Truax. EHV-UHV Transmission Systems. Transmission Line Reference Book: 345 kV and Above. Electric Power Research Institute, Palo Alto , Ca, 1982.

[Enk 84] Enk, J. O., M.M. Abromavage. Exposure to Low-Level Extremely Low Frequency Electromagnetic Fields Near Naval Communications Facilities. IEEE Journal of Oceanic Engineering OE-9(3):136-142, July, 1984.

[Fessard 74] Fessard, A. (editor). Handbook of Sensory Physiology. Volume 3: Electroreceptors and Other Specialized Receptors in Lower Vertebrates. Springer-Verlag, Berlin-Heidelberg, 1974.

[Fletcher $871 \quad$ Fletcher W. H., W.W. Shiu, T.A. Ishida, and W.R. Adey. A Modulated Microwave Field and Tumor Promoter May Inhibit Cell-Cell Communication and Cause an Increasd Sensitivity to Cytotoxic lymphokines and tumor necrosis factor. Technical Report, Contractors' Review Meeting, U.S. Dept. of Energy Office of Energy Storage and Distribution and the Electric Power Research Institute Health Studies Program, Kansas City, MO, 2-5 November, 1987.

[Florida 85] Florida Electric and Magnetic Fields Science Advisory Commission. Biological Effects of $60-\mathrm{Hz}$ Power Transmission Lines. Technical Report, Prepared for the Florida Department of Environmental Regulation, Tallahassee, FL, March, 1985.

[Florig 86] Florig, H.K. Population Exposure to Power-Frequency Fields - Concepts, Components, and Control.PhD thesis, Department of Engineering and Public Policy, Carnegie-Mellon University, Pittsburgh, PA, 1986.

[Florig 87a] Florig, H. K., 1. Nair, and M.G. Morgan. Briefing Paper1: Sources and Dosimetry of Power-Frequency Fields. Technical Report, Prepared for the Florida Department of Environmental Regulation under DER Contract SP1 17, March, 1987.

[Florig 87b] Florig, H.K, J.F. Hoburg, and M.G. Morgan. Electric Field Exposure from Electric Blankets. IEEE Transactions on Power Delivery PWRD-2(2):527-536, April, 1987.

[Free 81] Free, M. J., W. T. Kaune, R. D. Phillips, H.C.Cheng. Endocrinological Effects of Strong 60-Hz Electric Fields in Rats. BEMS2:105-121, 1981.

[Fulton 80] Fulton, J. P., S. Cobb, L. Preble, L. Leone and E. Forman. Electrical Wiring Configurations and Childhood Leukemia in Rhode Island. American J. ofEpiderniology111:292-296, 1980.

[Gauger 85] Gauger, J.R. Household Appliance Magnetic Field Survey.IEEE Transactions on Power Apparatus and Systems PAS-104(9), September, 1985.

[Gold 79] Gold E., L. Gordis, and J. Touascia. Risk Factors for Brain Tumors in Children. American Journal of Epidemiology 109:309-319, 1979. 
[Gona 87] Gona A. G., and M.C. Yu. Effects of 60-Hz Electric and Magnetics Fields on the Developing Rat Brain. Technical Report, Prepared for the New York State Power Lines Project, Wadsworth Labs, E-297, Empire State Plaza, Albany, New York, April, 1987.

[Goodman 86] Goodman, R. and A.S. Henderson. Sine Waves Enhance Cellular Transcription. Bioelectromagnetics 7(1):23-29, 1986.

[Goodman 871 Goodman, R., J. Abbott and A.S. Henderson. Transcriptional Patterns in the Sciara Coprophila. Bioelectromagnetics 8(1):1-8, 1987.

[Gould 82] Gould J.L. The Map Sense of Pigeons. Nature296:205-211, 1982.

[Graham 871 Graham, C., and H. D.Cohen. Influence of60 Hertz Fields on Human Behavior, Physiology, Biochemistry. Technical Report, Prepared for the New York State Power Lines Project, Wadsworth Labs, E-297, Empire State Plaza, Albany, New York, 1987.

[Graham 88] Graham, C., H. D. Cohen, M.R. Cook, M.M. Gerkovich and D.R. Riffle. Effects of /intermittent Exposure to $60 \mathrm{~Hz}$ Fields on Human Physiology. Technical Report, Contractors' Review Meeting, U.S. Dept. of Energy Office of Energy Storage and Distribution and the Electric Power Research Institute Health Studies Program, Phoenix, AZ, 30 October -3 November, 1988.

[Grandolfo 86] Grandolfo M., S.M. Michelson, and A. Rindi (editors). Biological Effects and Dosimetry of Static and ELF Electromagnetic Fields. Plenum Press, New York, 1986.

[Graves 85] Graves H.B. Effects of 60-Hz Fields on Chick Embryo and Chick Development, Growth and Behavior. Technical Report RP-1064, Electric Power Research Institute, Palo Alto, CA, 1985.

[Greenberg 85] Greenberg, R.S. and J.L. Shuster. Epidemiology of Cancer in Children. Epidemiologic Reviews 7:22-48, 1985.

[Gundersen 86] Gunderson R., B. Greenebaum and E. Goodman. Effects of 60-Hz Electromagnetic Flelds on Calcium Efflux and Neurotransmitter Release. Technical Report, Prepared for the New York State Power Lines Project, Wadsworth Labs, E-297, Empire State Plaza, Albany, New York, 1986.

[Guy 76] Guy, A.W., M.D. Webb, C.C. Sorensen. Determination of Power Absorption in Man Exposed to High Frequency Electromagnetic Fields by Thermographic Measurements on Scale Models. IEEE Transactions on Biomedical Engineering BME-23(5):361-371, 1976.

[Gyuk 88] Gyuk, I., U.S. Department of Energy, Washington, D.C. Personal communication, December. 1988

[Hansson 81] Hansson H.A.Lamellar bodies in Purkinje Nerve Cells Experimentally Induced by electric field. Brain Research 216:1 87-191, 1981.

[Harvey $871 \quad$ Harvey, S.M. Measurement of the Effect ofa 115-kV Transmission Line on the Electric and Magnetic Field Environment in and Around a Public School. Technical Report 87-237-K, Ontario Hydro Research Division, October 27, 1987.

[Hellman 85] Hellman K. B., P.P. Brewer, A.K. Fowler, B. Hellman and M.L. Swicord. The Effect of Electromagnetic Fields on Lymphocyte Function: Enhancement of Mitogenic Stimulation. Technical Report, Abstract, Seventh Annual Meeting of the Bioelectromagnetics Society, San Francisco, CA, June 16-20, 1985.

IHeroux 871 Heroux, P. 60-Hz Electric and Magnetic Fields Generated by a Distribution Network. Bioelectromagnetics 8(2):1 35-148, 1987.

[Hill 65] Hill A.B. The Environment and Disease: Association or Causation? Proceedings of the Royal Society of Medicine 58:295-300, 1965.

[Hilton 81] Hilton D.I. and R.D. Phillips. Growth and Metabolism of Rodents Exposed to $60-\mathrm{Hz}$ Electric Fields. Bioelectromagnetics 2(4):381-389, 1981. 
[Hjeresen 80] Hjeresen D. L., W.T.Kaune, J.R. Decker, and R.D. Phillips. Effects of 60-Hz Fields on Avoidance Behavior and Activity of Rats. Bioelectromagnetics 1:299-312, 1980.

[ICRP 75] International Commission on Radiological Protection. Number 23: Report of the Task Group on Reference Man. Pergamon Press, Elmsford, New York, 1975.

[IEEE 88] IEEE Magnetic Fields Task Force of the AC Fields Working Group of the Corona and

Field Effects Subcommittee of the Transmission and Distribution Committee. Magnetic

Fields from Electric Power Lines - Theory and Comparison to Measurements. Technical Report, Paper 88-WM-078-8 presented at the Winter Meeting of the IEEE Power Engineering Society, New York, NY, January 31- February 5, 1988.

[IUS86 86] International Utility Symposium on the Health Effects of Electric and Mangetic Fields: Research, Communication, Regulation. Session 4- Evaluation of Communications Programs, 1986.Available from Ontario Hydro, Toronto, Canada.

[Jacobs 84] Jacobs, P.D. and F.M.Dietrich . Measurements of Transmission Line Electric Fields in a Residential Environment. IEEE Transactions on Power Apparatus and Systems

PAS-103(8):2237-2243, August, 1984.

[Jaffe 83] Jaffe R. A., C.A. Lopresti, D.B. Carr and R.D. Phillips. Prenatal Exposure to 60-Hz Fields: Effect on the Development of the Visual-Evoked Response in Rats. Bioelectromagnetics 4:327-339, 1983.

[Juster 79] Juster, F.T., P. Courant, G.J. Duncan, J.P. Robinson, and F.P. Stafford. Time Use in Economic and Social Accounts 1975-1976. Technical Report ICPSR 7580, Survey Research Center, Institute for Social Research, University of Michigan, 1979.

[Kaune 85] Kaune,W.T. and R.D. Phillips. Dosimetry for Extremely Low-Frequency Electric Fields. In M. Grandolfo and S. Michelson, Jr. (editors), Biological Effects and Dosimetry of Static and ELF Electromagnetic Fields. Plenum Publisher, New York, NY, 1985.

[Kaune 86] Kaune, W.T. Physical Interaction of Humans and Animals with Power-Frequency Electric and Magnetic Fields. In W.E. Feero (editor), Panel Session on Biological Effects of Power Frequency Electric and Magnetic Fields, IEEE Publication Number 86 TH0139-6-PWR. IEEE Biological Effects of Power Frequency Electric and Magnetic Fields Working Group, 1986.

[Kaune 87] Kaune, W. T., R.G. Stevens, N.J. Callahan, R.K. Severson, and D.B. Thomas. Residential Magnetic and Electric Fields. In L.E. Anderson, B.J.Kelman, R.J. Weigel (editors), Interactions of Biological Systems with Static and ELF Electric and Magnetic Fields. Proceedings of 23rd Annual Hanford Life Sciences Symposium, October 2-4, 1984, Richland, Washington, 1987. Available from NTIS. Order CONF-841041.

[Kelly 86] Kelly, H.R. Experience and Lessons from Texas. Technical Report, Proceedings of the International Utility Symposium, September 16-19, Toronto, Canada, 1986. Available from Ontario Hydro, Ontario, Canada.

[Korobkova 72] Korobkova, V. P., Y.A. Morozov, M.D. Stolarov and Y.A. Yakub. Influence of the Electric Field in 500 and $750 \mathrm{kV}$ Switchyards on Maintenance Staff and Means for Its Protection. Technical Report 23-06, CIGRE paper, 1972.

[Krause 85] Krause, N. Exposure of People to Static and Time Variable Magnetic Fields in Technology, Medicine, Research, and Public Life - Dosimetric Aspects. Technical Report, Presented at the Symposium on Biological Effects of Static and Extremely Low Frequency Magnetic Fields, Neuherberg, Federal Republic of Germany, May 13-15, 1985.

[Lee 86] Lee J.M. Jr., J.H. Brunke, G. E.Lee, G.L. Reiner and F.L. Shon. Electrical and Biological Effects of Transmission Lines :A Review. Technical Report, U.S. Department of Energy, Bonneville Power Administration, Portland, Oregon, 1986. 
[Lee $871 \quad$ Lee, J. M., Bonneville Power Administration, Portland, Oregon. Personal communication, October. 1987

[Liboff 84] Liboff, A. R., T. Williams Jr., D.M. Strong, and R. Wistar Jr. Time-Varying Magnetic Fields: Effect on DNA Synthesis. Science 223:818-820, February 24, 1984.

[Liboff 86] Liboff A.R. Cyclotron Resonance in Membrane Transport. In Chiabrera A., C. Nicolini and H.P. Schwan (editors), Interaction between Electromagnetic Fields and Cells. Plenum Press, London, 1986.

[Lin 85] Lin R.S. , P. C. Dischinger, J. Conde and K.P. Farrell. Occupational Exposure to Electromagnetic Fields and the Occurrence of Brain Tumors : An Analysis of Possible Associations. Journal of Occupational Medicine 27:413-415, 1985.

[Lin 87] Lin R. Mortality Patterns Among Employees of Electric Power Company in Taiwan. Technical Report, Contractors' Review Meeting, U.S. Dept. of Energy Office of Energy Storage and Distribution and the Electric Power Research Institute Health Studies Program, Kansas City, MO, 2-5 November, 1987.

[Livingston 86] Livingston G. K., O.P. Gandhi, 1. Chatterjee, K. Witt, J. L. Roti. Reproductive Integrity of Mammalian Cells Exposed to $60-\mathrm{Hz}$ Electromagnetic Fields. Technical Report, Prepared for the New York State Power Lines Project, Appendix 6, Wadsworth Labs, E-297, Empire State Plaza, Albany, New York, 1986.

[Lovsund80 80] Lovsund, P. Biological Effects of Alternating Magnetic Fields with Special Reference to the Visual System. PhD thesis, Departments of Biomedical Engineering and Ophthalmology, Linkoping University, Linkoping, Sweden, 1980.

[Luben 82] Luben, R.A., C.D. Cain, M.C-Y. Chen, D.M. Rosen, and W.R. Adey. Effects of Electromagnetic Stimuli in Bone and Bone Cells in Vitro: Inhibition of Responses to Parathyroid Hormone by Low-energy, Low-frequency Fields. Proceedings of the National Academy of Sciences 79:4180-4184, ¡ 982.

[Lyle 83] Lyle, D. B., P. Schechter, W.R. Adey, and R.L. Lundak. Suppression of T-Lymphocyte Toxicity Following Exposure to Sinusoidally Alternating Magnetic Fields. Bioelectromagnetics 4:281-292, 1983.

[Lyle 86] Lyle D. B., R.D. Ayotte, A.R. Sheppard, and W.R. Adey. Proliferation of Myeloid Leukemia Cell Lines, and Allogeneic Toxicity in the Presence of 60-Hz Fields. 1986.Contractors' Review Meeting, U.S. Dept. of Energy Office of Energy Storage and Distribution and the Electric Power Research Institute Health Studies Program, Denver, CO, November, 1986.

ILymangrover 83] Lymangrover J. R., E. Keku, Y.J. Seto. 60-Hz Electric Field Alters the Steroidogenic Response of Rat Adrenal Tissue, In Vitro. Life Sciences 32:691-696, 1983.

ILymangrover 87] Lymangrover J. R., E. Keku, S.T. Hsieh, W.P.Dunlap, and Y.J. Seto. Direct PowerFrequency Electric Field Effects on Mammalian Endocrine Tissue. Environment/ Research 43:157-167, 1987.

IMaffeo 84] Maffeo S., M.W. Miller and E.L. Carstensen. Lack of Effects of Weak Low Frequency Electromagnetic Fields on Embryogenesis. Journal of Anatomy 139:613, 1984.

[Male 87] Male, J. C., W.T. Norris, and M.W. Watts. Exposure of People to Power-Frequency Electric and Magnetic Fields. In L.E. Anderson, B.J. Kelman, R.J. Weigel (editors), /interactions of Biological Systems with Static and ELF Electric and Magnetic Fields. Proceedings of 23rd Annual Hanford Life Sciences Symposium, October 2-4, 1984, Richland, Washington, 1987. Available from NTIS. Order CON F-841 041.

[Martucci 84] Martucci G, I., P.C. Gailey, and R.A. Tell. Investigation of Possible Effects of Weak, Pulsed Magnetic Fields on the Chick Embryo. Technical Report, Abstract, Sixth Annual Meeting of the Bioelectromagnetics Society, Atlanta, Gecrgia, July 15-19, 1984. 
[Maurizi 84] Maurizi C.P. Disorder of the Pineal Gland Associated with Depression, Peptic Ulcers and Sexual Dysfunction. Southern Medical Journa/77:151 6-1518, December, 1984.

[McClanahan 83] McClanahan B.J. and R.D. Phillips. The Influence of Elecatric Field Exposure on Bone Growth and Fracture Repair in Rats. Bioelectromagnetics 4:1 1-19, 1983.

[McDowall 83] McDowall M.E. Mortality of Persons Resident in the Vicinity of Electricity Transmission Facilities. Lancet 77:246, 1983.

[McLaughlin 87] McLaughlin J. K., H.S.R. Malker, W.J. Biot, B.K. Malker, B.J. Stone, J.A. Weiner, J.L. E. EricSon, and J.F. Fraumeni. Occupational Risks for Intracranial Glioma in Sweden. JNCl 78:253-257, February, 1987.

[Milham 82] Milham, S., Jr. Mortality from Leukemia in Workers Exposed to Electrical and Magnetic Fields. New England Journal of Medicine 307:249, 1982.

[Milham 85] Milham S. Silent Keys: Leukemia Mortality in Electricl Workers. Lancet 1:812, 1985.

[Miller 74] Miller, D.A. Electric and Magnetic Fields Produced by Commercial Power Systems. In Llaurado and Sances (editors), Biological and Clinical Effects of Low-Frequency Magnetic and Electric Fields, pages 62-70. CC Thomas, Springfield, MA, 1974.

[Minner $871 \quad$ Minner, D. The Top 100 Utilities' 1986 Operating Performance. Electric Light and Power, August, 1987.

[Morgan 83] Morgan, M. G., D.R. Lincoln, 1. Nair and H.K. Florig. An Exploration of Risk Assessment Needs and Opportunities for Possible Health Consequences from 50/60-Hz Electromagnetic Fields.. Internal report, Department of Engineering and Public Policy, Carnegie-Mellon University, November, 1983.

[Morgan 85] Morgan, M. G., H.K.Florig, 1. Nair, and D. Lincoln. Power-line Fields and Human Health. /EEE Spectrum 22(2):62-68, February, 1985.

[Morgan 87a] Morgan, M. G., H.K. Florig, 1. Nair, and G.L. Hester. Power Frequency Fields: The Regulatory Dilemma. Issues in Science and Technology Summer:81-91, 1987.

[Morgan 87b] Morgan, M. G., 1. Nair, H.K.Florig, and G.L. Hester. Briefing Paper 2: A Framework for Thinking About, and Making Regulatory Decisions About, Power-Frequency Electromagnetic Fields.

Technical Report, Prepared for the Florida Department of Environmental Regulation under DER Contract SP117, March, 1987.

[Morgan 87c] Morgan, M. G., H.K. Florig, 1. Nair, and G.L. Hester. Briefing Paper 3: Control Strategies for Power-Frequency Electromagnetic Fields Which Do Not Require a Measure of Dose. Technical Report, Prepared for the Florida Department of Environmental Regulation under DER Contract SP1 17, March, 1987.

[Morgan 87d] Morgan, M. G., H.K. Florig, 1. Nair, and G.L. Hester. Briefing Paper 4: Moving from General Policy Advice to Practical Regulations. Technical Report, Prepared for the Florida Department of Environmental Regulation under DER Contract SP1 17, March, 1987.

[Morgan 87e] Morgan, M. G., H.K. Florig, 1. Nair, and D.R. Lincoln. Some First Steps Toward Assessing Possible Health Risks from Exposure to 50/60-Hz Electromagnetic Fields. In L.E. Anderson, B.J.Kelman, R.J. Weigel (editors), /interactions of Biological Systems with Static and ELF Electric and Magnetic Fields. Proceedings of 23rd Annual Hanford Life Sciences Symposium, October 2-4, 1984, Richland, Washington, 1987. Available from NTIS. Order CONF-841041.

[Morgan 88] Morgan, M. G., H.K.Florig, 1. Nair, and G.L. Hester. Controlling Exposure to Transmission Line Electromagnetic Fields: A Regulatory Approach that is Compatible with the Available Science. Public Utilities Fortnightly, March 17, 1988.

[Morris 82] Morris J.E. and R.D. Phillips. Effects of $60-\mathrm{Hz}$ Electric Fields on Specific Humoral and Cellular Components of the Immune System. Bioelectromagnetics 3:341-349, 1982. 
[Morris 87] Morris J. E., M.E. Frazier and L.E. Anderson. Effects of 60-Hz Fields on Immune Response of Rats. Technical Report, Contractors' Review Meeting, U.S. Dept. of Energy Office of Energy Storage and Distribution and the Electric Power Research Institute Health Studies Program, Kansas City, MO, 2-5 November, 1987.

[MWN 84] . Pulsed Magnetic Fields: Conflicting Results. Microwave News 4, June, 1984.

[MWN 86] . EPA Closes Bioeffects Lab. Microwave News 6(5):2, September/October, 1986.

[MWN 87], . \$25 Million Power Line Award Reversed; Health Issue Affirmed. Microwave News 7(6):1, November/December, 1987.

[MWN 88a] . DOE EMF Bioeffects Budget Set at \$3 Million. Microwave News 8(5):8, September/October, 1988.

[MWN 88b] . CA EMF Health Effects Bill Signed into Law. Microwave News 8(5):7, September/October, 1988.

[MWN 88c] Budgets for EMF Research Are Growing at EPRI. Microwave News 8(2):6, March/April, 1988. -

[MWN 88d] \$66.5 Million Marcy-South Power Line Trial Underway. Microwave News 8(5):1, September/October, 1988.

[Myers 85] Myers A., R.A. Cartwright, J.A. Bonnell, J.C. Male and S.C. Cartwright. Overhead Power Lines and Childhood Cancer. Technical Report, Proceedings of the International Conference on Electric and Magnetic Fields in Medicine and Biology, 1985.

[Norris 87] Norris, W. T., J.C. Male, and B.J. Maddock. Magnetic and Electric Power Frequency Fields in the Home. In L.E. Anderson, B.J. Kelman, R.J. Weigel (editors), Interactions of Biological Systems with Static and ELF Electric and Magnetic Fields. Proceedings of 23rd Annual Hanford Life Sciences Symposium, October 2-4, 1984, Richland, Washington, 1987. Available from NTIS. Order CON F-841 041.

[NYSPLP $871 \quad$ New York State Powerlines Project Scientific Advisory Panel . Biological Effects of Power Line Fields. Technical Report, Prepared for the New York State Power Lines Project, Wadsworth Labs, E-297, Empire State Plaza, Albany, New York, 1987.

[Olin 85] Olin, R., D. Vagero, and A. Ahlbom. Mortality Experience of Electrical Engineers. British Journal of Industrial Medicine 42:211-212, 1985.

[Ossenkopp 86] Ossenkopp K.P. and D.P. Cain. ELF Low Intensity Magnetic Fields and Epilepsy. Technical Report, Prepared for the New York State Power Lines Project, Wadsworth Labs, E-297, Empire State Plaza, Albany, New York, 1986.

[Peterson 80] Peterson G.R. and S. Milham. Occupational Mortality in the State of California, 1959-61. Technical Report, National Institute for Occupational Safety and Health, Cincinnati, Ohio, 1980.

[Phillips 79] Phillips, R. D., M.F.Gillis, W.T. Kaune, D.D. Mahlum (editors). Biological Effects of Extreme/y Low Frequency Electromagnetic Fields. Technical Information Center, U.S. Department of Energy, 1979. Proceedings of the Eighteenth Hanford Life Sciences Symposium, October 16-18, 1978, Pacific Northwest Laboratory, Richland, Washington, CONF-781 016.

[Preston 82] Preston-Martin S., Henderson B.E. and J.M. Peters. Descriptive Epidemiology of Central Nervous System Neoplasms in Los Angeles County. Annals of the New York Academy of Sciences $381: 202-208,1982$.

[Quinlan 85] Quinlan W. J., D. Petrondas, N.Lebda, S. Pettit and S.M. Michelson. Neuroendocrine Parameters in the Rat Exposed to 60-Hz Electric Field. Bioelectromagnetics 6:381-389, 1985.

[Ragan 83] Ragan H. A., R.L. Buschbom, M.J. Pipes, R.D. Phillips and W.T. Kaune. Hematologic and Serum Chemistry Studies in Rats Exposed to 60-Hz. Bioelectromagnetics 4(3):79-90, 1983. 
[Rogers $871 \quad$ Rogers W.R. and H.D. Smith. Effects of Electric Fields on Posture and Position of Nonhuman Primates. Technical Report, Contractors' Review Meeting, U.S. Dept. of Energy Office of Energy Storage and Distribution and the Electric Power Research Institute Health Studies Program, Kansas City, MO, 2-5 November, 1987.

IRommereim 87] Rommereim, D. N., W.T.Kaune, R.L. Buschbom, R. L., R.D. Phillips, and M.R. Sikov. Reproduction and Development in Rats Chronically Exposed to $60-\mathrm{Hz}$ Electric Fields.

Bioelectromagnetics 8(3):243-258, 1987.

[Rozzell 74] Rozzell, T.C. Biological Research for Extremely Low Frequency Communications Systems. In J.G.Llaurado, A. Sances Jr., and J.H.Battocletti (editors), Biologic and Clinical Effects of Low-Frequency Magnetic and Electric Fields, chapter 7, pages 91-97. Charles C. Thomas, Springfield, Illinois, 1974.

[Russell 71] Russell D.S. and Rubinstein L.J. Pathology of Tumors of the Nervous System. Williams \& Wilkins, Baltimore, Maryland, 1971.

[Sahl 87] Sahl, J., Research Scientist, Southern California Edison Co., Rosemead, California. Personal communication, October. 1987

[Salzinger 87] Salzinger K. Behavioral Effects of ELF,. Technical Report, Prepared for the New York State Power Lines Project, Wadsworth Labs, E-297, Empire State Plaza, Albany, New York, 1987.

[Savitz 87a] Savitz, D.A. Case-Control Study of Childhood Cancer and Exposure to Electromagnetic Fields. Technical Report, Prepared for the New York State Power Lines Project, Albany, NY: Health Research Inc., 1987.

[Savitz 87b] Savitz D.A. and E.E. Cane. Leukemia and Occupational Exposure to Electromagnetic Fields : Review of Epidemiological Surveys. Journal of Occupational Medicine 29:47-51, 1987.

[Savitz 88] Savitz D. A., H.A. Wachtel, F. Barnes, E.M. John and and J.G. Tvrdik. Case-Control Study of Childhood Cancer and Exposure to 60-Hertz Magnetic Fields. American Journal of Epidemiology 128(1):21-38, 1988.

[Schwan 57] Schwan, H. P., and C.F. Kay. The Conductivity of Living Tissues. Annals of the New York Academy of Science 65:1007-1013, 1957.

[Sendaula 84] Sendaula, M., D.W. Hilson, R.C. Myers, L.G. Akens, and B. J. Woolery. Analysis of Electric and Magnetic Fields Measured Near TVA's 500-kV Transmission Lines. IEEE Transactions on Power Aparatus and Systems PAS-103(2):328-336, February, 1984.

[Sever 84] Sever, H., Center for Disease Control, Atlanta, GA. Personal communication. 1984

[Sheppard 83] Sheppard, A.R. "Biological Effects of High Voltage AC Transmission Lines" Report to the Montana Department of Natural Resources and Conservation, 1983.

[Sikov 87] Sikov M. R., D.N. Rommereim, J.L. Beamer, R.L. Buschbom, W.T. Kaune and R.D. Phillips. Developmental Studies of Hanford Miniature Swine Exposed to $60-\mathrm{Hz}$ Electric Fields. Bioelectromagnetics 8:229-242, 1987.

[Silva 88] Silva, J. M., N. Hummon, D. Rutter, and C. Hooper. Power-Frequency Magnetic Fields in the Home. Technical Report, Paper88-WM-101 -8 presented at the IEEE Power Engineering Society Winter Meeting, New York, New York, January 31-February 5, 1988.

[-- 85] Silva, M., N.P. Hummon, D. Huber, L.E. Zaffanella, and D.W. Deno. AC Field Exposure Study: Human Exposure to 60-Hz Electric Fields. Technical Report EA-3993, Prepared for the Electric Power Research Institute, April, 1985. 
[Simmons 86] Simmons, D. J., J. Wood, M. Wajuvajch, J. Poland and J.E. Russell. Effect of Magnetic Fields on Linear and Radial Bone Growth in Rats --A Circadian Study. Journal of Bioelectricity 5:187-199, 1986.

[Smith 87] Smith S. D., B.R. McLeod, A.R. Liboff and K. Cooksey. Calcium Cyclotron Resonance and Diatom Motility. Bioelectromagnetics 8:215-228, 1987.

[Snell 82] Snell K. (editor). Developmental Toxicology. Praeger Publishers, New York, 1982.

[Spiegel 76] Spiegel, R.J. ELF Coupling to Spherical Models of Man and Animals. IEEE Transactions on Biomedical Engineering BME-23(5), September, 1976.

[Spiegel 77] Spiegel, R.J. Magnetic Coupling to a Prolate Spheroid Model of Man. IEEE Transactions on Power Apparatus and Systems PAS-96(1):208-212, January/February, 1977.

[Stern 83] Stern S. and V.G.Laties, C.V. Stancampiano, C. Cox and J.O. deLorge. Behavioral Detection of $60-\mathrm{Hz}$ Electric Fields by Rats. Bioelectromagnetics 4:215-247, 1983.

[Stern 85] Stern S. and V.G. Laties. 60-Hz Electric Field Detection by Female Rats. Bioelectromagnetics 6:99-103, 1985.

[Stern 86] Stern F. B., R.A. Waxweiler, J.J. Beaumont. A Case-Control Study of Leukemia at a Naval Nuclear Shipyard. American Journal of Epidemiology 123:980-992, 1986.

[Stevens 87] Stevens R.G. Epidemiological Studies of Cancer and Residential Exposure to Electromagnetic Fields. Technical Report 21082-18, Prepared for the New York State Power Lines Project, Wadsworth Labs, Empire State Plaza, Albany, New York, 1987.

[Stuchly 83] Stuchly, M. A., D.W. Lecuyer and R.D. Mann . "Extremely Low Frequency Electromagnetic Emissions from Video Display Terminals and Other Devices". Health Physics 45(3):713-722, 1983.

[Sulzman 86] Sulzman F. M., and D.E. Murrish. Circadian Rhythms. Technical Report, Prepared for the New York State Power Lines Project, Wadsworth Labs, E-297, Empire State Plaza, Albany, New York, 1986.

[Sussman 88] Sussman, S., Manager, Non-lonizing Radiation Subprogram, Environment Division, 1988

[Tell 83] Electric Power Research Institute, Palo Alto, CA. Personal communication, December. Equipment, Calibration, and Selected Applications. In M. Grandolfo, S.M. Michelson, and A. Rindi (editors), Biological Effects and Dosimetry of Nonionizing Radiation. Plenum Publishing Company, New York, 1983.

[Thomas 86a] Thomas, J. R., J. Schrot, and A.R. Liboff. Low Intensity Magnetic Fields Alter Operant Behavior in Rats. Bioelectromagnetics 7(4):349-357, 1986.

[Thomas 86b] Thomas J.R. and J. Schrot. Investigation of Potential Behavioral Effects of Exposure to 60- $\mathrm{Hz}$ Electromagnetic Fields. Technical Report, Prepared for the New York State Power Lines Project, Wadsworth Labs, E-297, Empire State Plaza, Albany, New York, 1986.

[Thomas 87] Thomas T.L., P.D. Stolley, A. Stemhagen, E.T.H. Fontham, M.L. Bleecker,P.A. Stewart and R.N. Hoover. Brain Tumor Mortality Risk Among Men with Electrical and Electronics Jobs : A Case-Control Study. Journal of the National Cancer Institute 79:233-236, 1987.

[Tinga 73] Tinga, W.R. and S.0. Nelson. Dielectric Properties of Materials for Microwave Processing - Tabulated. Journal of Microwave Power 8(1):23-65, 1973.

[Tomenius 86] Tomenius, L. 50-Hz Electromagnetic Environments and the Incidence of Childhood Tumors in Stockholm County. Bioelectromagnetics 7:191-207, 1986. 
[Tornqvist 86] Tornqvist S., S. Norell, A. Ahlbom. Cancer in the Electric Power Industry. British Journal of Industrial Medicine 43:212-213, 1986.

[Ubeda 83] Ubeda A., J. Leal, M.A. Trillo, M.A. Jimenez and J.M. R. Delgado. Pulse Shape of Magnetic Fields Influences Chick Embryogenesis. Journal of Anatomy 137:513-536, 1983.

[USDOE 83] U.S. Department of Energy. Statistics of Private/y-Owned Electric Utilitites, 1981 Annual. Technical Report 130 E/EIA-0044(81), Energy Information Administration, 1983.

[Vagero 83] Vagero, D. and R. Olin. Incidence of Cancer in the Electronics Industry: Using the New Swedish Cancer Environment Registry as a Screening Instrument. British Journal of Industrial Medicine 40:188-192, 1983.

[Vagero 85] Vagero, D., A. Ahlbom, R. Olin, and S. Sahlsten. Cancer Morbidity among Workers in the Telecommunications Industry. British Journal of Industrial Medicine 42:191-195, 1985.

[Valentino 72] Valentine, A.R. A Small ELF Electric Field Probe. In IEEE International Electromagnetic Compatibility Symposium Record, pages 265-270. 1972.

[Vasquez 86] Vasquez B. J., L.E. Anderson, C. Lowery and W.R. Adey. Effects of 60 Hertz Fields on the Daily Rhythms of Opiate Receptors. Society for Neuroscience Abstracts 11:443, 1986.

[Walker 82] Walker C. F., D.L. Seitelman, J.H. McElharey, S.P. Muller, B. Hagadovn, Y.J. Seto. Effects of High Intensity 60-Hz Fields in Bone Growth. Journal of Bioelectricity 1:339-349, 1982.

[Wertheimer 79] Wertheimer, N. and E. Leeper. Electrical Wiring Configurations and Childhood Cancer. American Journal of Epidemiology 109:273-284, 1979.

[Wertheimer 82] Wertheimer, N. and E. Leeper. Adult Cancer Related to Electrical Wires Near the Home. International Journal of Epidemiology 11:345-355, 1982.

[West 86] WEST Associates. A Critical Review of the Scientific Literature on Low-Frequency Electric and Magnetic Fields: Assessment of Possible Effects on Human Health and Recommendations for Research. Technical Report, Southern California Edison Company, Rosemead, CA, 1986.

[Wever 74] Wever R. ELF-Effects on Human Circadian Rhythms. VLF ELectromagnetic Field Effects. Plenum Press, New York, 1974, pages 101-144.

[Wever 79] Wever R. The Circadian System of Man. Springer-Verlag, New York, 1979.

[WHO 84] World Health Organization, United Nations Environment Programme, and the International Radiation Protection Association. Environmental Health Criteria 35-

Extremely Low Frequency (ELF) Fields. Technical Report, World Health Organization, Geneva, Switzerland, 1984.

[Wilson 74] Wilson J.G. Environment and Birth Defects. Academic Press, Inc., New York, 1974.

[Wilson 81] Wilson, B. W., L.E. Anderson, D.1. Hilton, and R.D. Phillips. Chronic Exposure to 60-Hz Electric Fields: Effects on Pineal Function in the Rat. Bioelectromagnetics 2(4):371 -380, 1981,

[Wilson 83] Wilson, B. W., L.E. Anderson, D.1. Hilton, and R.D. Phillips. Erratum to 'Chronic Exposure to $60-\mathrm{Hz}$ Electric Fields: Effects on Pineal Function in the Rat', Bioelectromagnetics 2:371-380, 1981. Bioelectromágnetics 4(3):293, 1983.

[Winters 86] Winters W.D. Biological Functions of immunologically Reactive Human and Canine Cc//s Influenced by In Vitrc Exposure to 60-Hz Electric and Magnetic Fields. Technical Report, Prepared for the New York State Power Lines Project, Wadsworth Labs, E-297, Empire State Plaza, Albany, New York, September, 1986.

[Wolpaw 87] Wolpaw J. R., R.F. Seegal, R.1. Dowman and S. Satya-Murti. Chronic Effects of 60-Hz Electric and Magnetic Flelds on Primate Central System Function. Technical Report, Prepared for the New York State Power Lines Project, Wadsworth Labs, E-297, Empire State Plaza, Albany, New York, 1987. 
[Wright 82] Wright, W. E., J.M. Peters, and T.M. Mack. Leukaemia in Workers Exposed to Electrical and Magnetic Fields. The Lancet I(8303):1 160-1161, 1982. 


\section{Endnotes}

This section consists of notes that describe the background information of some of the aspects of biology and epidemiology central to the understanding of the ELF fields effect science.

\section{Note 1: The role of calcium In biological systems}

The flow of calcium ions $\left(\mathrm{Ca}^{+2}\right)$ across the cell membrane is an important means of transmitting signals from the exterior to the interior of the cell in all organisms. "Pumps" in the membrane maintain a 10,000fold difference between extracellular ( about 10-3 molar) and intracellular ( about $10^{-7}$ molar) free calcium ion concentrations. Most of the intracellular calcium is normally bound to other molecules or otherwise sequestered. Calcium is also present bound to the membranes to be released in the event of an appropriate triggering signal. Component molecules of the membrane (primarily those called called glycoproteins) have charged ends that attract and hold a layer of ions, principally calcium and hydrogen ions competing for the available sites. This layer of protein ends and ions (an ionic bilayer) is a major element that modulates the action of drugs and other chemicals.

\section{Note 2: Hormones}

Hormones which are produced by glands of the endocrine system play a major role in regulating the numerous functions of the body. The four general functions of the endocrine system are: maintenance of the intricately balanced biochemical and conditions in the body; response to everyday demands of physiologic and psychologic stress; integration of growth and development in the appropriate time sequence; and processes controlling sexual reproduction and fetal and newborn nourishment.

For some functions a multiplicity of hormones have to act in concert. For example, the sequence of hunger, and appetite production are controlled by a system of six hormones acting in sequence arising from different glands in the body. On the other hand, there are some hormones such as insulin, parathyroid hormone and epinephrine each of which performs multiple actions.

Because of their complexity, it is not simple to extrapolate observations of hormonal changes induced by ELF fields in in vitro cellular experiments to conclusions about end effects in the whole body. The effect of a hormone in the body depends on its interaction with a receptor which in turn depends on the circulating level of hormone present. There are basal and stimulated levels of secretion of hormones and the levels are also regulated by various feedback mechanisms. In vitro observation cannot simulate all the above processes that act together in the body.

The three major classes of hormones are; steroid, peptide and aminoacid. Cortisol which regulates appetite, and is an example of a steroid hormone while PTH and ACTH are peptide hormones.

Catecholamines are aminoacid hormones. The chemical nature and structure of the hormone determines how the hormone operates biochemically. All hormones interact with receptors on the cell membrane, and the combination initiates a transmembrane message which for most hormones is an activation of adenylate cyclase, an enzyme that is bound to the inner surface of the cell membrane. Adenylate cyclase 
then "sends" cyclic AMP (cAMP) which can widely circulate through the cell and is "the second messenger" that activates and regulates other enzymes through an intermediate enzyme called protein kinase. One of these, called protein kinase $C$, has been associated with the mechanism for cancer promotion. CAMP and calcium comprise the primary messenger system, both being released from stores on the cell membrane in response to messages.

Hormones that appear often in description of ELF effects are: the catecholamines, the serotonin 5-HT)- melatonin sequence, parathyroid hormone (PTH), ACTH, and growth factors (GF). These are briefly described below.

Catecholamines and the serotonin group belong to a class of hormones known as autacoids, or autopharmacologic agents and also as local hormones.

Catecholamines: Most prominent among the aminoacid hormones synthesized in the brain and the nervous system are the catecholamines dopamine, norepinephine and epinephine. As they are released from nerve endings during physiologic stimulation, they are called neurotransmitters. They have several functions and affect muscle, heart, liver, spleen, lung and brain physiology. They control blood pressure, heartbeats, some forms of headache, the basic metabolic rate, some psychic changes and several other rates. Deficient or excessive secretion of catecholamines or alterations of their action cause major physiological and psychological problems, Increased metabolism of catecholamines are also associated with neuroblastoma, a tumor originating in the neural crest, often during fetal development.

Serotonin, 5-hydroxytryptamine (5-HT) and Melatonin

Serotonin, first identified as a vasoconstrictor material, that appears in the serum when blood clots is the common name for the hormone whose active part is the complex 5-HT or 5-hydroxytryptamine: Serotonin is widely present in animal and plant tissue and has a wide array of regulatory action. $5-\mathrm{HT}$ is synthesized all over the body, from tryptophan which is present in many foods, notably milk. Receptors for serotonin are present in cell membranes all over the body, Serotonin stimulates or inhibits many of the muscles and nerves, depending on the amount and the phase of the organ in its function. It can stimulate or depress heartbeat, contract blood vessels and change blood pressure. Serotonin prevents clotting, and provides reflexes such as coughing or hyperventilation.

In humans, serotonin also serves as a chemical transmitter in the brain. Serotonin and its product melatonin influences sleep, perception of pain, psychological depression and social behavior.

Melatonin is also secreted by the pineal gland and is described in the endnote on circadian rhythms.

\section{Parathyroid hormone (PTH)}

PTH is a major hormone in the control of mineral (calcium, magnesium and phosphorus) metabolism. PTH and a group of other hormones, together with extracellular calcium, synergize and feedback on each other to regulate processes such as the growth and availability of calcium for conveying messages into the cells. Produced in the thyroid gland, PTH secretion in the cells is regulated primarily 
by calcium and cAMP. Calcium concentration in the extracellular fluid is the primary variable that is controlled by $\mathrm{PTH}$, which this determines the rate of resorption of Calcium in bones, kidney and intestine tissue.\}

\section{$\underline{\text { Adenocorticotropin }} \underline{(\mathrm{ACTH})}$}

ACTH is a hormone produced by the pituitary gland and is responsible for stimulating the secretion of the steroid hormone cortisol and related steroids by the adrenal gland. Cortisol levels in turn govern ACTH levels in the blood, and further ACTH production. The ACTH level is a function of the presence of cortisol or analogous steroids such as cortisone and aldosterone, the circadian rhythm and stress. The circadian variation of ACTH regulates that of cortisol which governs hunger and appetite, control of hypertension, and other functions; cortisone, which governs inflammatory response; and corticosterone which governs stress response, sexual development and other function. The "biologic clock" and stress work through the central nervous system to stimulate ACTH. The stimulation of cortisol by ACTH is mediated by cyclic AMP.

\section{Note 3: Stages In the formation of a tumor or cancer}

[Cole 87, Guddon 81, Berenblum 75, Trosko 83, Trosko 85]

For convenience in understanding the origins and development of a tumor or cancer, oncogenesis or carcinogenesis ${ }^{1}$ is usually thought of in terms of three stages : initiation, latent period and clinical manifestation.

Initiation is generally assumed to be a clear period in time when an agent damages the genetic material of a cell to produce changes that starts the tumor or cancer by changing the codes for production of essential biomolecules, or by inducing the production of molecules normally foreign to the organism at that stage of its life. As shown in Figure 1-1, reproduced from an article by Swenberg [Rice 79], this damage may be repaired depending on the repair mechanisms, the extent of damage and genetic susceptibility for repair.

During the latent period, the altered cells (also called "transformed"* or "neoplastic" cells) continue to grow and proliferate, to finally manifest themselves into detectable sizes as a "frank" or clinical tumor. Both external agents and epigenetic (or, extranuclear) events maybe important at this stage. An external agent may be a promoter, that is, it might enhance the effect of an initiator to further promote the transformation which may not otherwise not have been completed or for which the dose of the initiator was not enough.

The idea that tumor cancer development is a multistage process came from the pioneering experiments of Rous and his colleagues [Friedewald 44]. They found that virus-induced skin tumors that regressed after a period of time could be made to grow again if the skin is physically stressed or by application of irritants such as croton oil. Rous et al then concluded that the tumor cells could exist in a latent state and could be "promoted" by appropriate agents. They named these stages initiation and promotion. Berenblum then did an elegant series of experiments on mouse skin tumors showing clearly

\footnotetext{
1"onco" is a prefix denoting a benign or malignant tumor, while "carcino" denotes a cancer which may be a malignant tumor
} 

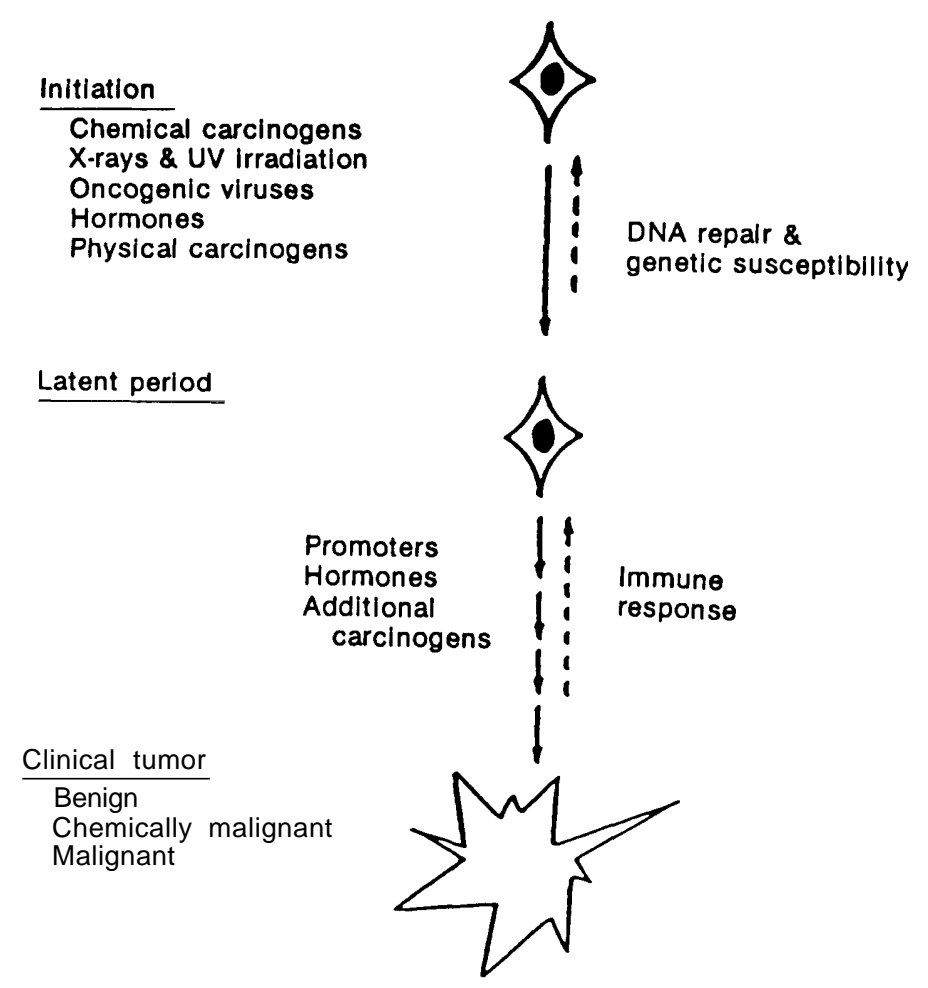

Figure 1: Stages in the development of a tumor or cancer [Rice 79]

that there were substances that could not initiate cancer but could promote its growth [Berenblum 41]. Although these experiments were performed almost forty years ago, and vigorous research has continued since, we still do not have a clear delineation of the processes and mechanisms involved in the stages of oncogenesis or carcinogenesis.

Initiation and growth alone are enough to induce cancer. Promotion by a separate or the same agent is not necessary. A single appropriately high dose of certain carcinogens have been shown to induce cancer in animals.

Initiation and promotion has now been widely studied in chemical carcinogenesis after the identification of chemicals such as urethane which are primarily initiators and components of croton oil that have only a promoting activity. The classic promoter used as a standard because of its potency is a chemical isolated from croton oil, 12-o-tetradecanoyl phorbol-13-acetate, or, TPA.

External agents may also affect the growth of the cancer by enhancing cell growth and proliferation processes or by having the properties to enhance those of a transformed cell in preference to a normal cell. Epigenetic events that maybe important during the latent period in increasing the rate of growth are: the hormone and immunologic status of the body; the stage of tissue differentiation especially if the affected body is that of a fetus or neonate; and metabolic competence in general, or in particular with respect to certain agents that are promoters or growth enhancers.

Trosko and Chang have developed models of some aspects of multistage carcinogenesis and reviewed the subject in detail [Trosko 83, Trosko 85]. They have hypothesized that in some cases at least, promotion occurs when a cell escapes control by the normalizing regulating molecular signals from its communicating neighbors. 
circadian regulation was a powerful selection agent in evolution. Submammalian vertebrates exhibit endogenous circadian rhythmicity. That is, a submammalian pineal separated from the organism, continues its cycle of function given the proper LD cycle. This is because in birds and other lower vertebrates, the pineal itself contains photoreceptive cells, a function that has been separated to the SCN in mammals.

The pineal contains a multitude of critical biochemical. Among the most studied of these are:

1. norepinephrine, serotonin and dopamine( neurotransmitters that transmit excitatory and inhibitory signals in the central and peripheral nervous system)

2. histamine (a chemical mediator found in connective tissues throughout the body that causes blood vessels to dilate facilitating the access of serum proteins, antibodies and white blood cells to the location as needed to respond to injury and infection),

3. melatonin ( a hormone that regulates sexual function and development, among other things) which has been most widely studied, and other related indolamines.

4. the inhibitory neurotransmitter gamma amino butyric acid (GABA). In addition, there are a number of other pineal hormones.

It is evident from the above that an environmental agent that affects the pineal could have important biological effects. The most striking aspect of indolamine synthesis in the pineal is the marked circadian rhythms in the concentrations and therefore in the modulations of the synthetic processes. Light is the single most significant environmental stimulus guiding these. Pinealectomy experiments in fish, amphibians, reptiles and birds have established several interesting rules about the circadian rhythmsetting role of the pineal.

Pineal-ScN interaction in mammals is distinctly different from these other vertebrates and work on pinealectomy on mammals has provided only sparse and inconclusive data, The pineal in the mammal appears to be a feedback modulator of the circadian activity. Disorder of the pineal gland has been associated with a range of symptomatic disorders such as psychological depression and sexual dysfunction [Maurizi 84]. Low levels of melatonin have been found in people with certain types of cancer and melatonin has been administered to cancer patients to inhibit cancer growth.

\section{Note 5: Epidemiological Studies [Savitz 87, Rothman 86, Aldrich 85]}

Epidemiological studies have the notable advantage that they are studies of human populations and are, therefore their results, if clear-cut, should be directly applicable in public health decision-making without the animal to human extrapolation problems faced by experimental studies. However, because there are ethical and practical limitations on the extent to which epidemiological studies can be designed as experiments, their results are often inconclusive, the main problems being confounding effects, uncertainties about exposures and concurrent behaviors and exposures of the subjects, recall biases and, in the case of weak agents with non-specific effects, which maybe the case for ELF field exposure, that of getting enough numbers in the study to provide statistical significance or" power" to the study.

Epidemiology has really begun to emerge as a sound methodology only in the last three decades, with several epidemiological studies by Health departments in the U.S. such as the Framingham Heart Study initiated in 1949. The Heart study has been instrumental in providing much of our knowledge about cardiovascular disease and health habits and still continues to yield results., after 35 years. Other 
Tumor promotion itself is likely to be a multistep process including some or all of the following :

1. Inhibition of the intercellular communication which normally restricts cell proliferation beyond the healthy regime for the organ,

2. Changing or blocking of normal regulatory processes within the cell, or,

3. Affecting gene expression (i.e., the manufacture of the appropriate proteins) through enzyme induction or inhibition. This may lead to the production of too much of the normally occurring proteins or that of altered proteins.

From the above, it is clear that the most obvious limitation of an in vitro result in studying mechanisms of carcinogenesis is the fact that carcinogenesis is an in vivo process that is a combination of physiologic factors, immune responses, pharmacokinetics, and metabolic effects.

Two facts about the cellular level experiments involving field exposure are worth noting. Ornithine decarboxylase production is increasingly being used as a biochemical marker of some types of cancers. [] It has also been noted that membrane modulators which control or inhibit calcium efflux or cause an internal redistribution of calcium will act as stimulants of promotion or as promoters of cancer. (Trosko85b)

Note 4: Circadian Systems and the Pineal Gland [Relter 84]

While all the biology of the circadian and other timing systems is not well understood as yet, research in the last two decades have brought considerable understanding of some of the elements of the system. It is believed that the system has several elements that work in some logical sequence. Prime among these are : transducers that sense cues of time; pacemakers which keep time; and, mediators that are neural and biochemical pathways which transfer appropriate information to the respective systems. Under general constant conditions, circadian rhythms are "free-running", that is, they show periods close to 24 hours. Environmental stimuli can be used to re-synchronize or "entrain" these cycles to other periods within some limited range of periods. A stimulus that is usually used in experiments is the "light-dark" (LD) cycle, which can be used to entrain both periods and phases of biological activity

The specialized organs that have a major role in the organization of circadian rhythms in mammals are the suprachiasmatic nucleus (SCN) and the pineal gland. The SCN is a nucleus located in the hypothalamus ( the region of the brain that is the main regulator of the endocrine system), close to the base of the optic nerve. The SCN receives direct input from the retina that conveys information about light and dark and continues receiving this information, independent of conscious light-dark perception. The pineal, so named for its pine cone-like structure, is a tiny gland, weighing only about 0.15 gram in the human, at the base of the brain. The pineal receives its regulatory signals directly from the SCN via a neural pathway. In mammals, when there is no light input, pineal rhythms still persist; but these are no longer entrained to the external LD cycle but rather to a free-running rhythm set by the SCN which is believed to be the only internal clock that drives the pineal in the mammal. Any signal that cues the SCN and therefore the pineal are termed "zeitgebers" or, time givers. The LD cycle is the most important natural zeitgeber for the pineal.

The pineal is one of the most important regulatory organs in the body, one that has existed through millenia of evolution. The pineal gland acts as a neurochemical transducer, that is, it converts information about the level of environmental lighting into neurochemical activity. Thus it plays an important role in controlling hormone systems, although its full function is not yet known. It is believed that the protection of certain biochemical processes from lethal solar ultraviolet radiation by confining them to the night via 
triumphs of epidemiology have been in establishing the connection between smoking and health, lowlevel ionizing radiation and leukemia, and maternal ingestion of DES (diethylstilbestrol) and cancer in female offspring.

Because it is a science in its infancy, epidemiology still faces some disagreement and confusion about definition of measures. Considerable time and effort are needed to make the basic measurements in epidemiology which track the disease incidence. Getting data from people is often difficult, very timeand resource-intensive, and hampered by problems of non-cooperation, population mobility and privacy.

Rothman points out that the theoretical foundation of epidemiology lies in statistics but that some of the methods that have become incorporated into epidemiology are not theoretically sound in biological applications while they make sense in other applications in statistics. Statistical hypothesis testing is such a method that was evolved for agricultural and quality control experiments with very uniform and discrete results. The criteria for significance in disease definition are often arbitrary and the concept of significance not always meaningful.

\section{Cause and Effect in Epidemiology}

Many of the public health effects result from interaction of several factors in a biological system so that the "single cause"-to-"observed effect" often used in the generalization of epidemiological studies to public health protection measures is too simplistic. "Sufficient cause" for a public health effect often consists of many components acting in concert or in a definite or random sequence. Strength of each cause, interaction among causes and proportion of disease due to specific causes all combine to produce an epidemiological effect. Often the biologic factors underlying any or all of these are unknown and this makes interpretation of associations difficult.

Because of the lack of knowledge of biological mechanisms and individual variation, when we are faced with a decision of making a public health decision about an agent we assign an average value of risk of a particular health effect from the agent to everyone in the exposed population while in reality there are some individuals who have no risk at all of contracting that particular effect from that agent. For example, smoking does not necessarily cause lung cancer in everyone who smokes. What we know is we face enough of a whole set of causes of lung cancer that removing smoking, the one known component cause makes sense from a public health point of view.

\section{Types of Epidemiologic Studies}

Epidemiologic studies may be designed experiments as in the case of chemical, field and community intervention trials; or, as is more often the case, they may be studies of selected populations who have been exposed to the agent of interest. Obviously, all studies involving exposures to environmental agents with potential or unknown harmful effects have to be of the latter type.

A major difference between follow-up and case-control studies is that the subjects are chosen according to some exposure of the agent of interest in follow-up studies and according to a disease or end point in case control studies. As a time interval is allowed to elapse as part of the study in the 
follow-up case, these are called longitudinal studies. A case-control study is essentially a cross-sectional study, looking at a sample of the cross-section of diseased population at a particular point in time. Obviously, etiology of a disease is better studied with the longitudinal follow-up studies.

\section{Measures of Effect in Epidemiology}

Epidemiology may measure effects on the absolute level in terms of incidence rate, cumulative incidence or prevalence; and on a relative scale by several measures described below. When one potential cause is to be studied, a useful measure to work at is the attributable proportion for which the disease incidence can be said to have arisen from exposure to the agent as sufficient cause. Strictly speaking, attributable risk should be calculated only if the causation has been established.

The ELF field studies have all measured the possible effect in terms of the relative measures, Relative Risk (RR), Odds Ratio (OR), Proportional Mortality Ratio (PM R), and Standardized Mortality Ratio (SMR). A couple of early studies calculated Proportional Incidence Ratio (PIR) or Proportional Registration Ratio (PRR).

Relative measures essentially take the ratio of the incidence rate or difference in incidence rate in exposed population to ratio among unexposed or less exposed. If $I_{0}$ and $I_{1}$ are respectively the ratio of incidence among unexposed (or less exposed) and among exposed, the relative risk is simply the ratio $I_{1}$ $/ 1_{0}$. The relative risk is also referred to as the relative incidence, or rate ratio.

In case-control studies, cases are those who became ill during a time period and the controls area fraction of exposed and unexposed (all undiseased) persons. If the sampling for controls is random and an equal fraction of exposed and unexposed persons have been sampled, the relative risk is the exposure odds ratio, that is, the exposure odds among cases to exposure odds among controls, usually called the Odds Ratio.

When death certificate or mortality statistics are used, the proportional mortality ratio or PMR is taken as a measure of the effect of an exposure. PMR is the ratio of the exposed subjects who died of a specific cause to the unexposed subjects who died of the same cause. PMR values have to be interpreted carefully for a specified cause because if the subjects are exposed to a spectrum of causes and other relevant agents, a high PMR might reflect the preventive effect of an agent in the unexposed population rather than the causative effect of the agent on the exposed population.

Instead of mortality statistics, if registration or other incidence data are available, Proportional Incidence ratio (PIR) or Proportional Registration Ratio (PRR) can be calculated. 


\section{References to Endnotes}

[Aldrich 85] Aldrich, T.E. and C.E. Easterly. Handbook of Epidemiological Methods, with Special Emphasis on Extremely Low Frequency Electromagnetic Fields. Technical Report ORNL-6237, Prepared for the Department of Energy, Oak Ridge National Laboratory, Oak Ridge, Tennessee, 1985.

[Berenblum 41] Berenblum 1. The Mechanism of Carcinogenesis : A Study of the Significance of Carcinogenic Action and Related Phenomena. Cancer Research 1 :807-812, 1941.

[Berenblum 75] Berenblum 1. Sequential Aspects of Chemical Carcinogenesis: Skin. Cancer: $A$ Comprehensive Treatise. Plenum Press, New York, 1975, pages 323-344.

[Cole 87] Cole P. An Epidemiologic Perspective on Electromagnetic Fields and Cancer. Prepared for the Florida Department of Environmental Regulation, March. 1987.

[Friedewald 44] Friedewald W.D. and P. Rous. The Initiating and Promoting Elements in Tumor Production: An Analysis of the Effects of Tar, Benzopyrene, and Methylcholantrene on Rabbit Skin. Journal of Experimental Medicine 80:101 -122, 1944.

[Guddon 81] Guddon R.W. Cancer Biology. Oxford University Press, New York, 1981.

[Maurizi 84] Maurizi C.P. Disorder of the Pineal Gland Associated with Depression, Peptic Ulcers and Sexual Dysfunction. Southern Medical Journa/77:151 6-1518, December, 1984.

[Reiter 84] Reiter R.J. (editor). The Pineal Gland. Raven Press, New York, 1984.

[Rice 79] J.M. Rice. Perinatal Carcinogenesis. Technical Report, National Cancer [Institute Monograph No.51, Washington, D. C., 1979.

[Rothman 86] Rothman K.J. Modern Epidemiology. Little Brown Company, New York, 1986.

[Savitz 87] Savitz, D.A. Case-Control Study of Childhood Cancer and Exposure to Electromagnetic Fields. Technical Report, Prepared for the New York State Power Lines Project, Albany, NY: Health Research Inc., 1987.

[Trosko 83] Trosko J.E. and C.C. Chang. Role of Intercellular Communication in Tumor Promotion. Tumor Promotion and Carcinogeensis in vitro. CRC Press, Boca raton, Florida, 1983.

[Trosko 85] Trosko J.E. and C.C. Chang. Implications for Risk Assessment of Genotoxic and Non-Genotoxic Mechanisms in Carcinogenesis. Methods for Estimating Risk of Chemical Injury: Human and Non-Human Biota and Ecosystems. Academic Press, New York, 1985. 


\section{Other Related OTA Reports}

- Electric Power Wheeling \& Dealing: Technological Considerations for Increasing Competition. Explores the technical requirements for introducing greater competition into the operation and planning of the electric power industry, with particular emphasis on the reliability and operation of the transmission network. E-409, 4/89; $276 \mathrm{p}$.

Free summary available.

GPO stock \#052-003-01 153-1\$12.00

- New Electric Power Technologies: Problems and Prospects for the 1990s. Examines the development of a series of new technologies encouraged by the Federal Government that offer greater flexibility to utilities. Technologies to be examined include: utility-controlled load management; fuel cells; advanced coal- and gascombustion; photovoltaics; and solar thermal, wind, and geothermal power. E-246, $7 / 85 ; 340 \mathrm{p}$.

Free summary available. GPO stock \#052 -003-01005-4 \$12.00

NTIS order \#PB 86-121 746/AS

- Nuclear power in an Age of Uncertainty. Examines the future of nuclear power in this country; describes the current technological, economic, financial, public perception, and regulatory problems facing the domestic nuclear industry; identifies how various changes might affect the industry's future. E-216, 2/84; $296 \mathrm{p}$.

NTIS order \#PB 84-183 953/AS

NOTE: Reports are available through the U.S. Government Printing Office, Superintendent of Documents, Washington, DC 20401-9325, (202) 783-3238; and/or the National Technical Infer mation service, 5285 Port Royal Road, Springfield, VA 22161-0001, (703) 487-4650. 